\title{
First low-frequency Einstein@Home all-sky search for continuous gravitational waves in Advanced LIGO data
}

\begin{abstract}
B. P. Abbott et al.
(LIGO Scientific Collaboration and Virgo Collaboration)

(Received 29 June 2017; revised manuscript received 14 September 2017; published 8 December 2017)

We report results of a deep all-sky search for periodic gravitational waves from isolated neutron stars in data from the first Advanced LIGO observing run. This search investigates the low frequency range of Advanced LIGO data, between 20 and $100 \mathrm{~Hz}$, much of which was not explored in initial LIGO. The search was made possible by the computing power provided by the volunteers of the Einstein@Home project. We find no significant signal candidate and set the most stringent upper limits to date on the amplitude of gravitational wave signals from the target population, corresponding to a sensitivity depth of $48.7[1 / \sqrt{\mathrm{Hz}}]$. At the frequency of best strain sensitivity, near $100 \mathrm{~Hz}$, we set $90 \%$ confidence upper limits of $1.8 \times 10^{-25}$. At the low end of our frequency range, $20 \mathrm{~Hz}$, we achieve upper limits of $3.9 \times 10^{-24}$. At $55 \mathrm{~Hz}$ we can exclude sources with ellipticities greater than $10^{-5}$ within $100 \mathrm{pc}$ of Earth with fiducial value of the principal moment of inertia of $10^{38} \mathrm{~kg} \mathrm{~m}^{2}$.
\end{abstract}

DOI: 10.1103/PhysRevD.96.122004

\section{INTRODUCTION}

In this paper we report the results of a deep all-sky Einstein@Home [1] search for continuous, nearly monochromatic gravitational waves (GWs) in data from the first Advanced LIGO observing run (O1). A number of all-sky searches have been carried out on initial LIGO data, [2-15], of which $[2,3,7,9,14]$ also ran on Einstein@Home. Einstein@Home is a distributed computing project which uses the idle time of computers volunteered by the general public to search for GWs.

The search presented here covers frequencies from $20 \mathrm{~Hz}$ through $100 \mathrm{~Hz}$ and frequency derivatives from $-2.65 \times$ $10^{-9} \mathrm{~Hz} / \mathrm{s}$ through $2.64 \times 10^{-10} \mathrm{~Hz} / \mathrm{s}$. A large portion of this frequency range was not explored in initial LIGO due to lack of sensitivity. By focusing the available computing power on a subset of the detector frequency range, this search achieves higher sensitivity at these low frequencies than would be possible in a search over the full range of LIGO frequencies. In this low-frequency range we establish the most constraining gravitational wave amplitude upper limits to date for the target signal population.

\section{LIGO INTERFEROMETERS AND THE DATA USED}

The LIGO gravitational wave network consists of two observatories, one in Hanford (Washington) and the other

*Full author list given at the end of the Letter.

Published by the American Physical Society under the terms of the Creative Commons Attribution 4.0 International license. Further distribution of this work must maintain attribution to the author(s) and the published article's title, journal citation, and DOI. in Livingston (Louisiana) separated by a 3000-km baseline [16]. The first observing run (O1) [17] of this network after the upgrade towards the Advanced LIGO configuration [18] took place between September 2015 and January 2016. The Advanced LIGO detectors are significantly more sensitive than the initial LIGO detectors. This increase in sensitivity is especially significant in the low-frequency range of $20 \mathrm{~Hz}$ through $100 \mathrm{~Hz}$ covered by this search: at $100 \mathrm{~Hz}$ the $\mathrm{O} 1$ Advanced LIGO detectors are about a factor 5 more sensitive than the Initial LIGO detectors during their last run (S6 [19]), and this factor becomes $\approx 20$ at $50 \mathrm{~Hz}$. For this reason all-sky searches did not include frequencies below $50 \mathrm{~Hz}$ on initial LIGO data.

Since interferometers sporadically fall out of operation ("lose lock") due to environmental or instrumental disturbances or for scheduled maintenance periods, the data set is not contiguous and each detector has a duty factor of about $50 \%$. To remove the effects of instrumental and environmental spectral disturbances from the analysis, the data in frequency bins known to contain such disturbances have been substituted with Gaussian noise with the same average power as that in the neighboring and undisturbed bands. This is the same procedure as used in [3]. These bands are identified in the Appendix.

\section{THE SEARCH}

The search described in this paper targets nearly monochromatic gravitational wave signals as described for example by Eqs. (1)-(4) of [9]. Various emission mechanisms could generate such a signal, as reviewed in Sec. IIA of [15]. In interpreting our results we will consider a spinning compact object with a fixed, nonaxisymmetric $\ell=m=2$ mass quadrupole, described by an equatorial ellipticity $\varepsilon$. 
We perform a stack-slide type of search using the global correlation transform (GCT) method [20-22]. In a stackslide search the data is partitioned in segments, and each segment is searched with a matched-filter method [23]. The results from these coherent searches are combined by summing the detection statistic values from the different segments, one per segment $\left(\mathcal{F}_{i}\right)$, and this determines the value of the core detection statistic:

$$
\overline{\mathcal{F}}:=\frac{1}{N_{\text {seg }}} \sum_{i=1}^{N_{\text {seg }}} \mathcal{F}_{i} .
$$

The "stacking" part of the procedure is the summing, and the "sliding" (in parameter space) refers to the fact that the $\mathcal{F}_{i}$ that are summed do not all come from the same template.

Summing the detection statistic values is not the only way to combine the results from the coherent searches; see for instance $[4,24,25]$. Independently of the way that this is done, this type of search is usually referred to as a "semicoherent search." Important variables for this type of search are the coherent time baseline of the segments $T_{\text {coh }}$, the number of segments used $N_{\text {seg }}$, the total time spanned by the data $T_{\mathrm{obs}}$, the grids in parameter space, and the detection statistic used to rank the parameter space cells. For a stack-slide search in Gaussian noise, $N_{\text {seg }} \times 2 \overline{\mathcal{F}}$ follows a chi-squared distribution with $4 N_{\text {seg }}$ degrees of freedom, $\chi_{4 N_{\text {seg }}}^{2}$. These parameters are summarized in Table I. The grids in frequency and spin-down are each described by a single parameter, the grid spacing, which is constant over the search range. The same frequency grid spacings are used for the coherent searches over the segments and for the incoherent summing. The spin-down spacing for the incoherent summing, $\delta \dot{f}$, is finer than that used for the coherent searches, $\delta \dot{f}_{c}$, by a factor $\gamma$. The notation used here is consistent with that used in previous observational papers $[2,3]$.

The sky grid is approximately uniform on the celestial sphere projected on the ecliptic plane. The tiling is a hexagonal covering of the unit circle with hexagons' edge length $d$ :

TABLE I. Search parameters rounded to the first decimal figure. $T_{\text {ref }}$ is the reference time that defines the frequency and frequency derivative values.

\begin{tabular}{lc}
\hline \hline Parameter & Value \\
\hline$T_{\text {coh }}$ & $210 \mathrm{hr}$ \\
$T_{\text {ref }}$ & $1132729647.5 \mathrm{GPS} \mathrm{s}$ \\
$N_{\text {seg }}$ & 12 \\
$\delta f$ & $8.3 \times 10^{-7} \mathrm{~Hz}$ \\
$\delta f_{c}$ & $1.3 \times 10^{-11} \mathrm{~Hz} / \mathrm{s}$ \\
$\gamma$ & 100 \\
$m_{\text {sky }}$ & $1 \times 10^{-3}$ \\
\hline \hline
\end{tabular}

$$
d\left(m_{\mathrm{sky}}\right)=\frac{1}{f} \frac{\sqrt{m_{\mathrm{sky}}}}{\pi \tau_{E}},
$$

with $\tau_{E} \simeq 0.021 \mathrm{~s}$ being half of the light travel time across the Earth and $m_{\text {sky }}$ a constant which controls the resolution of the sky grid. The sky grids are constant over $5 \mathrm{~Hz}$ bands and the spacings are the ones associated through Eq. (2) to the highest frequency in each $5 \mathrm{~Hz}$. The resulting number of templates used to search $50 \mathrm{mHz}$ bands as a function of frequency is shown in Fig. 1.

This search leverages the computing power of the Einstein@Home project, which is built upon the BOINC (Berkeley Open Infrastructure for Network Computing) architecture [26-28]: a system that exploits the idle time on volunteer computers to solve scientific problems that require large amounts of computer power. The search is split into work units (WUs) sized to keep the average Einstein@Home volunteer computer busy for about $8 \mathrm{CPU}$ hours. Each WU performs $1.5 \times 10^{11}$ semicoherent searches, one for each of the templates in $50 \mathrm{mHz}$ band, the entire spin-down range and 118 points in the sky. Out of the semicoherent detection statistic values computed for the $1.5 \times 10^{11}$ templates, it returns to the Einstein@ Home server only the highest 10000 values. A total of $1.9 \times 10^{6}$ WUs are necessary to cover the entire parameter space. The total number of templates searched is $3 \times 10^{17}$.

\section{A. The ranking statistic}

Two detection statistics are used in the search: $\hat{\beta}_{\mathrm{S} / \mathrm{GLtL}}$ and $2 \overline{\mathcal{F}} . \hat{\beta}_{\mathrm{S} / \mathrm{GLtL}}$ is the ranking statistic which defines the

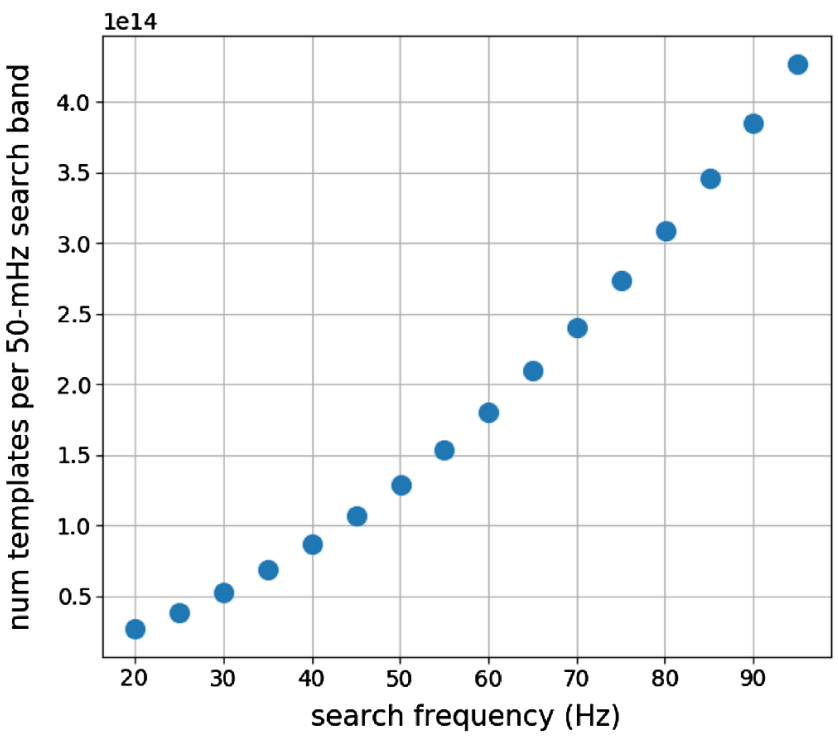

FIG. 1. Number of searched templates in $50 \mathrm{mHz}$ band as a function of frequency. The sky resolution increases with frequency causing the variation in the number of templates. $N_{f} \times N_{\dot{f}} \sim 1.3 \times 10^{9}$, where $N_{f}$ and $N_{\dot{f}}$ are the number of $f$ and $\dot{f}$ templates searched in $50 \mathrm{mHz}$ bands. The total number of templates searched between 20 and $100 \mathrm{~Hz}$ is $3 \times 10^{17}$. 
top-candidate list; it is a line- and transient-robust statistic that tests the signal hypothesis against a noise model which, in addition to Gaussian noise, also includes single-detector continuous or transient spectral lines. Since the distribution of $\hat{\beta}_{\mathrm{S} / \mathrm{GLtL}}$ is not known in closed form even in Gaussian noise, when assessing the significance of a candidate against Gaussian noise, we use the average $2 \mathcal{F}$ statistic over the segments, $2 \overline{\mathcal{F}}$ [23]; see Eq. (1). This is in essence, at every template point, the log-likelihood of having a signal with the shape given by the template versus having Gaussian noise.

Built from the multi- and single-detector $\hat{\mathcal{F}}$ statistics, $\hat{\beta}_{\mathrm{S} / \mathrm{GL} t \mathrm{~L}}$ is the $\log _{10}$ of $\hat{B}_{\mathrm{S} / \mathrm{GLtL}}$, the full definition of which is given by Eq. (23) of [29]. This statistic depends on a few tuning parameters that we describe in the remainder of the paragraph for the reader interested in the technical details: A transition-scale parameter $\hat{\mathcal{F}}_{*}^{(0)}$ is used to tune the behavior of the $\hat{\beta}_{\mathrm{S} / \mathrm{GLtL}}$ statistic to match the performance of the standard average $2 \overline{\mathcal{F}}$ statistic in Gaussian noise while still statistically outperforming it in the presence of continuous or transient single-detector spectral disturbances. Based on injection studies of fake signals in Gaussian-noise data, we set an average $2 \overline{\mathcal{F}}$ transition scale of $\hat{\mathcal{F}}_{*}^{(0)}=$ 65.826. According to Eq. (67) of [30], with $N_{\text {seg }}=12$ this $2 \overline{\mathcal{F}}$ value corresponds to a Gaussian false-alarm probability of $10^{-9}$. Furthermore, we assume equal-odds priors between the various noise hypotheses ("L" for line, "G" for Gaussian, "tL" for transient line).

\section{B. Identification of undisturbed bands}

Even after the removal of disturbed data caused by spectral artifacts of known origin, the statistical properties of the results are not uniform across the search band. In what follows we concentrate on the subset of the signalfrequency bands having reasonably uniform statistical properties, or containing features that are not immediately identifiable as detector artifacts. This comprises the large majority of the search parameter space.

Our classification of "clean" versus "disturbed" bands has no pretense of being strictly rigorous, because strict rigor here is neither useful nor practical. The classification serves the practical purpose of discarding from the analysis regions in parameter space with evident disturbances and must not dismiss detectable real signals. The classification is carried out in two steps: an automated identification of undisturbed bands and a visual inspection of the remaining bands.

An automatic procedure, described in Sec. II F of [31], identifies as undisturbed the $50-\mathrm{mHz}$ bands whose maximum density of outliers in the $f-\dot{f}$ plane and average $2 \overline{\mathcal{F}}$ are well within the bulk distribution of the values for these quantities in the neighboring frequency bands. This procedure identifies 1233 of the $160050-\mathrm{mHz}$ bands as undisturbed. The remaining 367 bands are marked as potentially disturbed, and in need of visual inspection.

A scientist performs the visual inspection by looking at various distributions of the $\hat{\beta}_{\mathrm{S} / \mathrm{GLtL}}$ statistic over the entire sky and spin-down parameter space in the 367 potentially disturbed $50-\mathrm{mHz}$ bands. She ranks each band with an integer score 0,1,2 ranging from "undisturbed" (0) to "disturbed" (2). A band is considered "undisturbed" if the distribution of detection statistic values does not show a visible trend affecting a large portion of the $f-\dot{f}$ plane. A band is considered "mildly disturbed" if there are outliers in the band that are localized in a small region of the $f-\dot{f}$ plane. A band is considered "disturbed" if there are outliers that are not well localized in the $f-\dot{f}$ plane.

Figure 2 shows the $\hat{\beta}_{\mathrm{S} / \mathrm{GLtL}}$ for each type of band. Figure 3 shows the $\hat{\beta}_{\mathrm{S} / \mathrm{GLtL}}$ for a band that harbors a fake signal injected in the data to verify the detection pipelines. In the latter case, the detection statistic is elevated in a small region around the signal parameters.

Based on this visual inspection, $1 \%$ of the bands between 20 and $100 \mathrm{~Hz}$ are marked as "disturbed" and excluded from the current analysis. A further $6 \%$ of the bands are marked as "mildly disturbed." These bands contain features that cannot be classified as detector disturbances without further study; therefore, these are included in the analysis.

Figure 4 shows the highest values of the detection statistic in half-Hz signal-frequency bands compared to the expectations. The set of candidates from which the highest detection statistic values are picked does not include the $50-\mathrm{mHz}$ signal-frequency bands that stem entirely from fake data, from the cleaning procedure, or that were marked as disturbed. Two $50-\mathrm{mHz}$ bands that contained a hardware injection [32] were also excluded, as the high amplitude of the injected signal caused it to dominate the list of candidates recovered in those bands. In this paper we refer to the candidates with the highest value of the detection statistic as the loudest candidates.

The highest expected value from Gaussian noise over $N_{\text {trials }}$ independent trials of $2 \overline{\mathcal{F}}$ is determined ${ }^{1}$ by numerical integration of the probability density function given, for example, by Eq. (7) of [33]. Fitting to the distribution of the highest $2 \overline{\mathcal{F}}$ values suggests that $N_{\text {trials }} \simeq N_{\text {templ }}$, with $N_{\text {templ }}$ being the number of templates searched.

The $p$ value for the highest $2 \overline{\mathcal{F}}$ measured in any half- $\mathrm{Hz}$ band searched with $N_{\text {trials }}$ independent trials is obtained by integrating the expected noise distribution $\left(\chi_{4 N_{\text {seg }}}^{2}\right.$ given in Sec. III) between the observed value and infinity, as done in Eq. (6) of [33]. The distribution of these $p$ values is shown in Fig. 5 and it is not consistent with what we expect from Gaussian noise across the measured range. Therefore, we cannot exclude the presence of a signal in this data based on this distribution alone, as was done in [3].

\footnotetext{
${ }^{1}$ After a simple change of variable from $2 \overline{\mathcal{F}}$ to $N_{\text {seg }} \times 2 \overline{\mathcal{F}}$.
} 


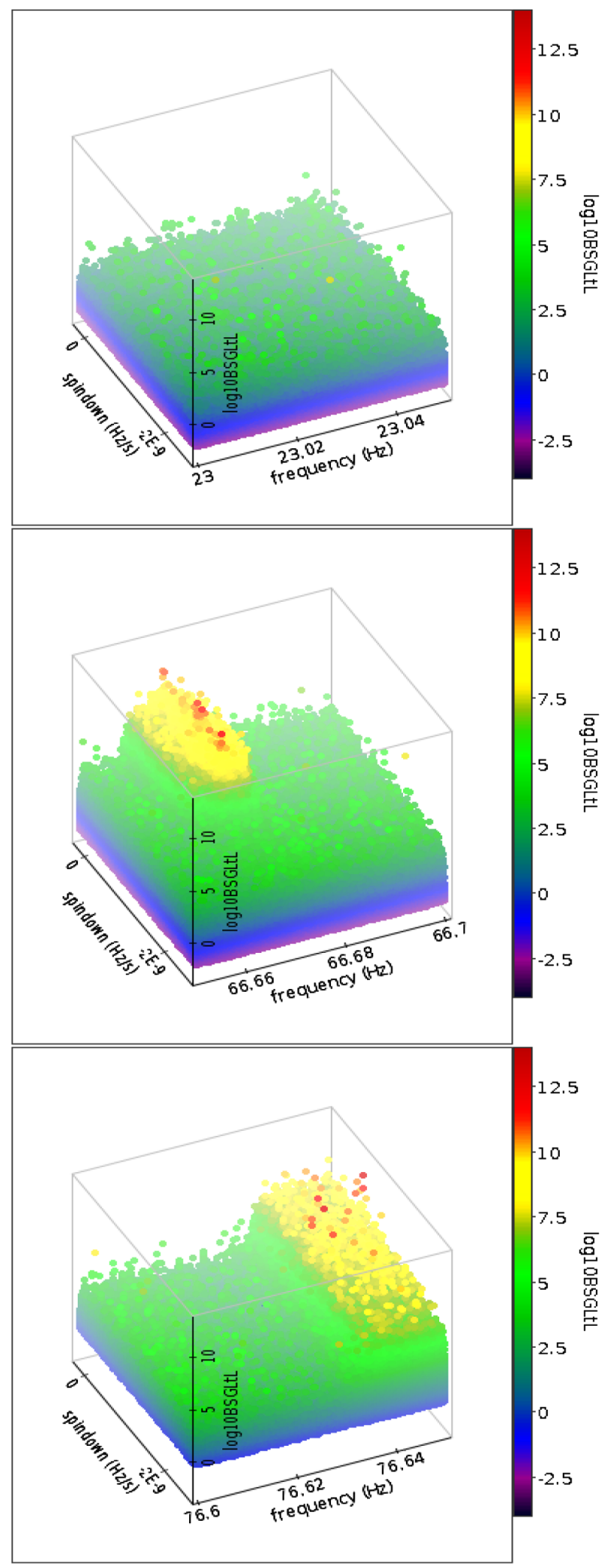

FIG. 2. On the vertical axis and color-coded is the $\hat{\beta}_{\mathrm{S} / \mathrm{GLtL}}$ in three $50-\mathrm{mHz}$ bands. The top band was marked as "undisturbed." The middle band is an example of a "mildly disturbed band." The bottom band is an example of a "disturbed band."

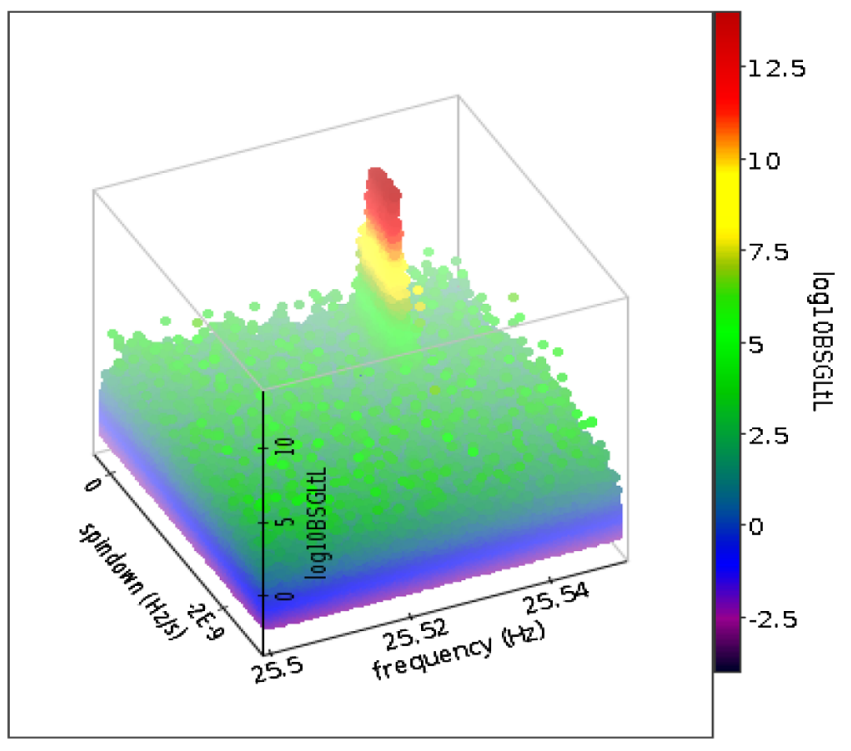

FIG. 3. This is an example of an "undisturbed band" but containing a fake signal. On the $z$ axis and color coded is the $\hat{\beta}_{\mathrm{S} / \mathrm{GLtL}}$.

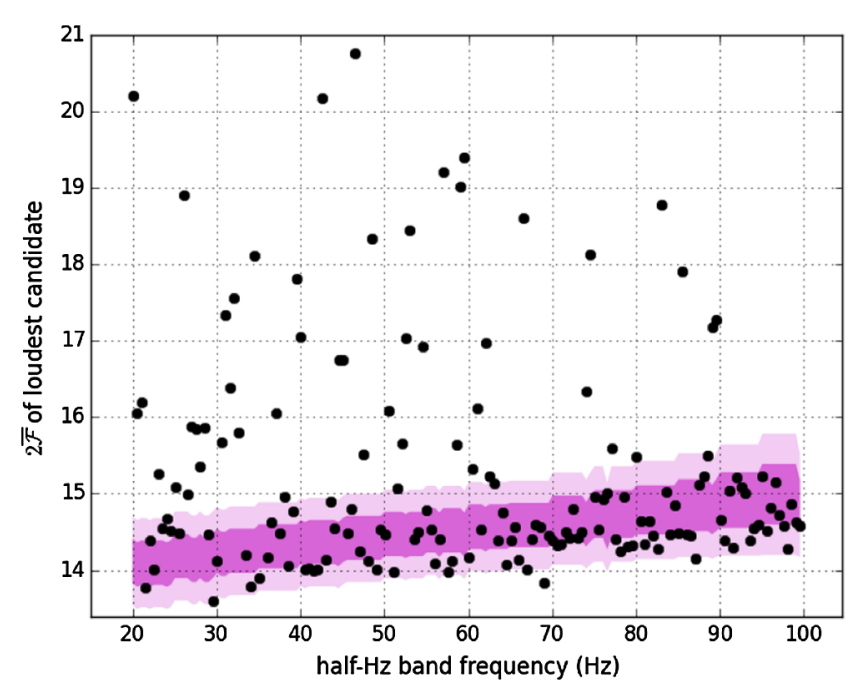

FIG. 4. Highest $2 \overline{\mathcal{F}}$ value (also referred to as the $2 \overline{\mathcal{F}}$ of the loudest candidate) in every half- $\mathrm{Hz}$ band as a function of band frequency. Since the number of templates increases with frequency, so does the highest $2 \overline{\mathcal{F}}$. The highest expected $2 \overline{\mathcal{F}} \pm$ $1 \sigma(2 \sigma)$ over $N_{\text {trials }}$ independent trials is indicated by the darker (faded) band. Two half-mHz bands have $2 \overline{\mathcal{F}}$ values greater than the axes boundaries. The half-Hz bands beginning at $33.05 \mathrm{~Hz}$ and $35.55 \mathrm{~Hz}$ have loudest $2 \overline{\mathcal{F}}$ values of 159 and 500 , respectively, due to features in the $33.3 \mathrm{~Hz}$ and $35.75 \mathrm{~Hz} 50-\mathrm{mHz}$ bands which were marked "mildly disturbed" in the visual inspection.

\section{HIERARCHICAL FOLLOW UP}

Since the significance of candidates is not consistent with what we expect from Gaussian noise only, we must investigate "significant" candidates to determine if they are 


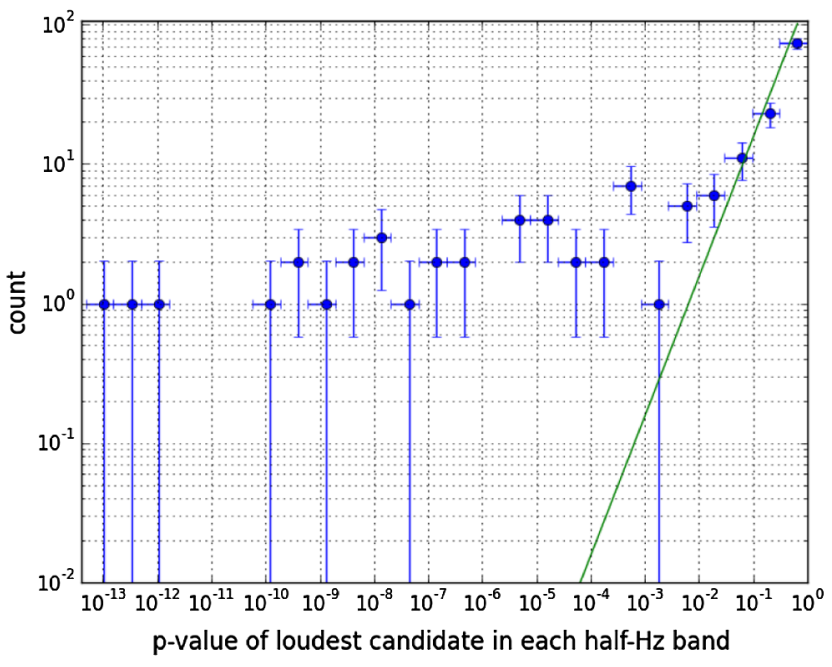

FIG. 5. Distribution of $p$ values, with binomial uncertainties, for the highest detection statistic values measured in half-Hz bands (circles) and expected from pure Gaussian noise (line). We note that the measured $p$ values for the highest $2 \overline{\mathcal{F}}$ in the $33.05 \mathrm{~Hz}$ and $35.55 \mathrm{~Hz}$ bands are not shown because they are outside of the $x$ axis boundaries.

produced by a signal or by a detector disturbance. This is done using a hierarchical approach similar to what was used for the hierarchical follow-up of subthreshold candidates from the Einstein@Home S6 all-sky search [2].

At each stage of the hierarchical follow-up a semicoherent search is performed, the top ranking candidates are marked and then searched in the next stage. If the data harbors a real signal, the significance of the recovered candidate will increase with respect to the significance that it had in the previous stage. On the other hand, if the candidate is not produced by a continuous-wave signal, the significance is not expected to increase consistently over the successive stages.

The hierarchical approach used in this search consists of four stages. This is the smallest number of stages within which we could achieve a fully coherent search, given the available computing resources. Directly performing a fully coherent follow-up of all significant candidates from the all-sky search would have been computationally unfeasible.

\section{A. Stage 0}

We bundle together candidates from the all-sky search that can be ascribed to the same root cause. This clustering step is a standard step in a multistage approach [2]: Both a loud signal and a loud disturbance produce high values of the detection statistic at a number of different template grid points, and it is a waste of compute cycles to follow up each of these independently.

We apply a clustering procedure that associates together multiple candidates close to each other in parameter space, and assigns them the parameters of the loudest among them, the seed. We use a new procedure with respect to [2]
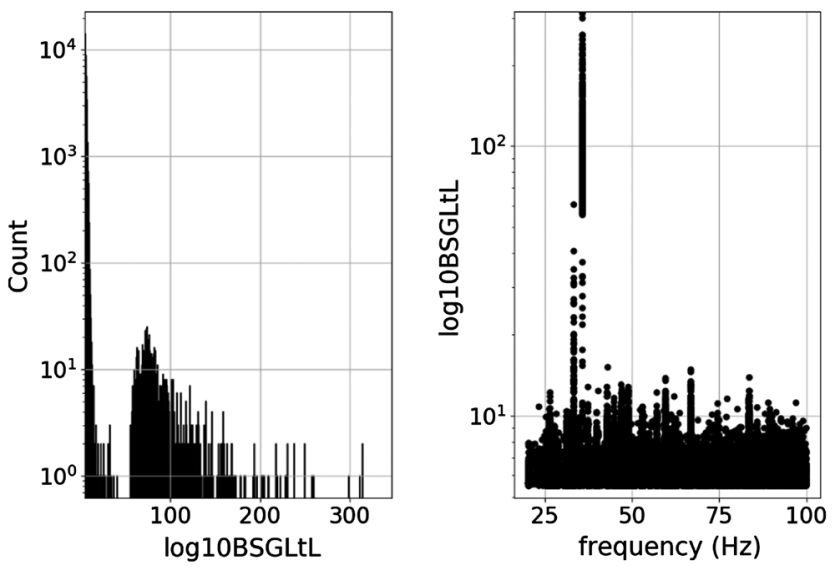

FIG. 6. Candidates that are followed up in stage 1: the distribution of their detection statistic values $\hat{\beta}_{\mathrm{S} / \mathrm{GLtL}}$ (left) and their distribution as a function of frequency (right).

that adapts the cluster size to the data and checks for consistency of the cluster volume with what is expected from a signal [34]. A candidate must have a $\hat{\beta}_{\mathrm{S} / \mathrm{GLtL}}>5.5$ to be a cluster seed. This threshold is chosen such that only a handful of candidates per $50 \mathrm{mHz}$ would be selected if the data were consistent with Gaussian noise. In this search, there are $15 \times 10^{6}$ candidates with $\hat{\beta}_{\mathrm{S} / \mathrm{GLtL}}>5.5$. A lower threshold of $\hat{\beta}_{\mathrm{S} / \mathrm{GLtL}}>4.0$ is applied to candidates that can be included in a cluster. If a cluster has at least two occupants (including the seed), the seed is marked for follow-up. In total, 35963 seeds are marked for follow-up. The $\hat{\beta}_{\mathrm{S} / \mathrm{GLtL}}$ values of these candidates are shown in Fig. 6 as well as their distribution in frequency.

Monte Carlo studies, using simulated signals added into the data, are conducted to determine how far from the signal parameters a signal candidate is recovered. These signals are simulated at a fixed strain amplitude for which most have $\hat{\beta}_{\mathrm{S} / \mathrm{GLtL}} \gtrsim 10.0$. We find that 1282 of 1294 signal candidates recovered after clustering (99\%) are recovered within

$$
\left\{\begin{array}{l}
\Delta f= \pm 9.25 \times 10^{-5} \mathrm{~Hz} \\
\Delta \dot{f}= \pm 4.25 \times 10^{-11} \mathrm{~Hz} / \mathrm{s} \\
\Delta \text { sky } \simeq 4.5 \text { sky grid points }
\end{array}\right.
$$

of the signal parameters. This confidence region ${ }^{2}$ defines the parameter space around each candidate which will be searched in the first stage of the hierarchical follow-up. For weaker signals the confidence associated with this uncertainty region decreases. For signals close to the threshold used here, namely with $\hat{\beta}_{\mathrm{S} / \mathrm{GLtL}}$ between 5.5 and 10 , the

\footnotetext{
${ }^{2}$ We pick $99 \%$ confidence rather than, say, $100 \%$, because to reach the $100 \%$ confidence level would require an increase in a containment region too large for the available computing resources.
} 
TABLE II. Search parameters for each stage. The follow-up stages are stages 1,2, and 3. Also shown are the parameters for stage 0 , taken from Table I.

\begin{tabular}{lrrccrc}
\hline \hline & $T_{\text {coh }}$ hr & $N_{\text {seg }}$ & $\delta f \mathrm{~Hz}$ & $\delta \dot{f}_{c} \mathrm{~Hz} / \mathrm{s}$ & \multicolumn{1}{c}{$\gamma$} & $m_{\text {sky }}$ \\
\hline Stage 0 & 210 & 12 & $8.3 \times 10^{-7}$ & $1.3 \times 10^{-11}$ & 100 & $1 \times 10^{-3}$ \\
Stage 1 & 500 & 5 & $6.7 \times 10^{-7}$ & $2.9 \times 10^{-12}$ & 80 & $8 \times 10^{-6}$ \\
Stage 2 & 1260 & 2 & $1.9 \times 10^{-7}$ & $9.3 \times 10^{-13}$ & 30 & $1 \times 10^{-6}$ \\
Stage 3 & 2512 & 1 & $6.7 \times 10^{-8}$ & $9.3 \times 10^{-14}$ & 1 & $4 \times 10^{-7}$ \\
\hline \hline
\end{tabular}

detection confidence only drops by a few percent (see bottom panel of Fig. 7 and last row of Table II in [34]).

\section{B. Stage 1}

In this stage we search a volume of parameter space [Eqs. (3)] around each cluster seed. We fix the run time per candidate to be $4 \mathrm{hr}$ on an average CPU of the ATLAS computing cluster [35]. This yields an optimal search setup having a coherent baseline of $500 \mathrm{hr}$, with 5 segments and the grid spacings shown in Table II. We use the same ranking statistic as the original search, $\hat{\beta}_{\mathrm{S} / \mathrm{GLtL}}$, with tunings updated for $N_{\text {seg }}=5$.

For the population of simulated signals that passed the previous stage, stage 0,1268 of $1282(99 \%)$ are recovered within the uncertainty region

$$
\left\{\begin{array}{l}
\Delta f= \pm 1.76 \times 10^{-5} \mathrm{~Hz} \\
\Delta \dot{f}= \pm 9.6 \times 10^{-12} \mathrm{~Hz} / \mathrm{s} \\
\Delta \text { sky } \simeq 0.23 \Delta \text { sky }^{\text {stage } 0} .
\end{array}\right.
$$
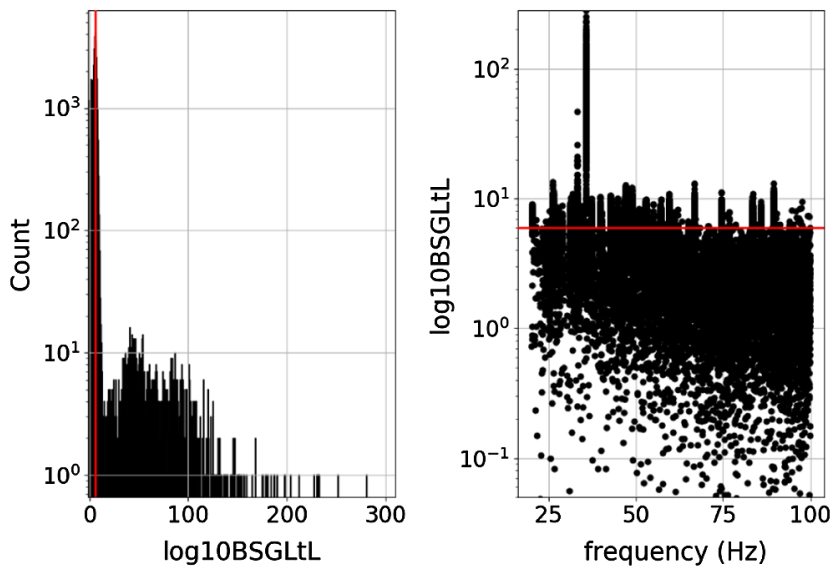

FIG. 7. Detection statistic of the loudest candidate from each stage 1 search: the distribution of their detection statistic values $\hat{\beta}_{\mathrm{S} / \mathrm{GLtL}}$ (left) and their distribution as a function of frequency (right). 411 candidates have $\hat{\beta}_{\mathrm{S} / \mathrm{GLtL}}$ values lower than the axes boundaries on the right plot. The red line marks $\hat{\beta}_{\mathrm{S} / \mathrm{GLtL}}=6.0$ which is the threshold at and above which candidates are passed on to stage 2 .
From each of the 35963 follow-up searches we record the most significant candidate in $\hat{\beta}_{\mathrm{S} / \mathrm{GLtL}}$. The distribution of these is shown in Fig. 7. A threshold at $\hat{\beta}_{\mathrm{S} / \mathrm{GLtL}}=6.0$, derived from Monte Carlo studies, is applied to select the candidates to consider in the next stage. There are 14456 candidates above this threshold.

\section{Stage 2}

In this stage we search a volume of parameter space [Eqs. (4)] around each candidate from stage 1 . We fix the run time per candidate to be $4 \mathrm{hr}$ on an average CPU of the ATLAS computing cluster [35]. This yields an optimal search setup having a coherent baseline of $1260 \mathrm{hr}$, with 2 segments and the grid spacings shown in Table II. We use a different ranking statistic from the original search, because with 2 segments the transient line veto is not useful. Instead we use the ranking statistic $\hat{\beta}_{\mathrm{S} / \mathrm{GL}}:=\log _{10} \hat{B}_{\mathrm{S} / \mathrm{GL}}$, introduced in [30] and previously used in [3], with tunings updated for $N_{\mathrm{seg}}=2$.

For the population of signals that passed the previous stage, 1265 of 1268 (>99\%) are recovered within the uncertainty region

$$
\left\{\begin{array}{l}
\Delta f= \pm 8.65 \times 10^{-6} \mathrm{~Hz} \\
\Delta \dot{f}= \pm 7.8 \times 10^{-12} \mathrm{~Hz} / \mathrm{s} \\
\Delta \text { sky } \simeq 0.81 \Delta \text { sky }^{\text {stage } 1}
\end{array}\right.
$$

From each of the follow-up searches we record the most significant candidate in $\hat{\beta}_{\mathrm{S} / \mathrm{GL}}$. The distribution of these is shown in Fig. 8. A threshold at $\hat{\beta}_{\mathrm{S} / \mathrm{GL}}=6.0$ is applied to determine what candidates to consider in the next stage. There are 8486 candidates above threshold.
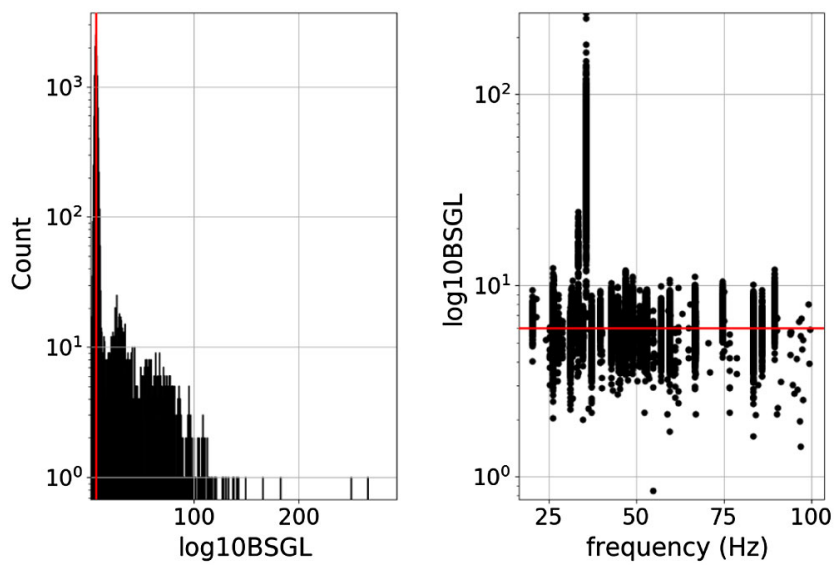

FIG. 8. Detection statistic of the loudest candidate from each stage 2 search: the distribution of their detection statistic values $\hat{\beta}_{\mathrm{S} / \mathrm{GL}}$ (left) and their distribution as a function of frequency (right). The red line marks $\hat{\beta}_{\mathrm{S} / \mathrm{GL}}=6.0$ which is the threshold at and above which candidates are passed on to stage 3 . 


\section{Stage 3}

In this stage we search a volume of parameter space [Eqs. (5)] around each candidate. We perform a fully coherent search, with a coherent baseline of $2512 \mathrm{hr}$. The grid spacings are shown in Table II. We use the same ranking statistic as the previous stage, $\hat{\beta}_{\mathrm{S} / \mathrm{GL}}$, with tunings updated for $N_{\text {seg }}=1$.

For the population of signals that passed the previous stage, 1265 of $1265(>99 \%)$ are recovered within the uncertainty region

$$
\left\{\begin{array}{l}
\Delta f= \pm 7.5 \times 10^{-6} \mathrm{~Hz} \\
\Delta \dot{f}= \pm 7 \times 10^{-12} \mathrm{~Hz} / \mathrm{s} \\
\Delta \text { sky } \simeq 0.99 \Delta \text { sky }^{\text {stage } 2} .
\end{array}\right.
$$

This uncertainty region assumes candidates are within the uncertainty regions shown in Eqs. (3), (4), and (5) for each of the corresponding follow-up stages. It is possible that a strong candidate which is outside these uncertainty regions would be significant enough to pass through all follow-up stages. In this case the uncertainty on the signal parameters would be larger than the uncertainty region defined in Eq. (6).

From each of the follow-up searches we record the most significant candidate in $\hat{\beta}_{\mathrm{S} / \mathrm{GL}}$. The distribution of these is shown in Fig. 9. A threshold at $\hat{\beta}_{\mathrm{S} / \mathrm{GL}}=6.0$ is applied to determine what candidates require further study. There are 6349 candidates above threshold. Many candidates appear to be from the same feature at a specific frequency. There are 57 distinct narrow frequency regions at which these 6349 candidates have been recovered.
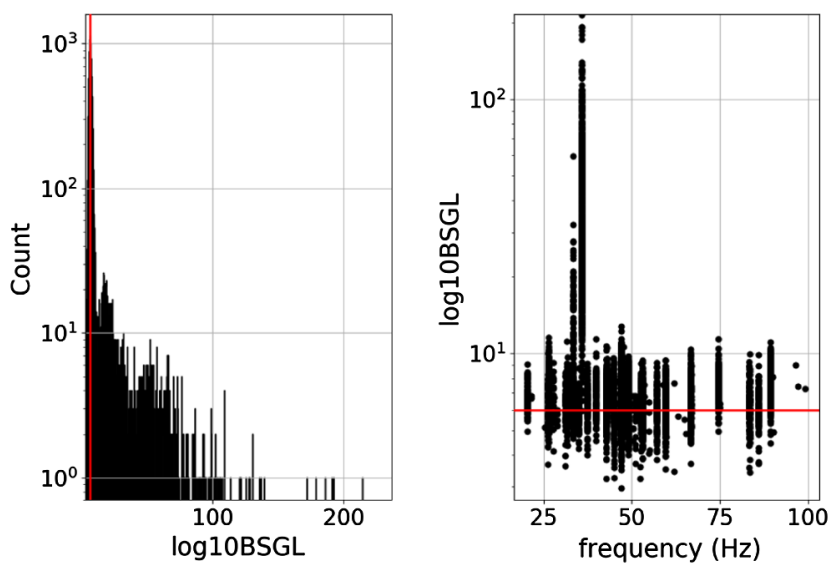

FIG. 9. Detection statistic of the loudest candidate from each stage 3 search: the distribution of their detection statistic values $\hat{\beta}_{\mathrm{S} / \mathrm{GL}}$ (left) and their distribution as a function of frequency (right). The red line marks $\hat{\beta}_{\mathrm{S} / \mathrm{GL}}=6.0$ which is the threshold below which candidates are discarded.

\section{E. Doppler modulation off veto}

We employ a newly developed Doppler modulation off (DM-off) veto [36] to determine if the surviving candidates are of terrestrial origin. When searching for $\mathrm{CW}$ signals, the frequency of the signal template at any point in time is demodulated for the Doppler effect from the motion of the detectors around the Earth and around the Sun. If this demodulation is disabled, a candidate of astrophysical origin would not be recovered with the same significance. In contrast, a candidate of terrestrial origin could potentially become more significant. This is the basis of the DM-off veto.

For each candidate, the search range of the DM-off searches includes all detector frequencies that could have contributed to the original candidate, accounting for $\dot{f}$ and Doppler corrections. The $\dot{f}$ range includes the original all-sky search range, and extends into large positive values of $\dot{f}$ to allow for a wider range of detector artifact behavior.

For a candidate to pass the DM-off veto it must be that its $2 \mathcal{F}_{\text {DM-off }} \leq 2 \mathcal{F}_{\text {DM-off }}^{\text {thr }}$. The $2 \mathcal{F}_{\text {DM-off }}^{\text {thr }}$ is picked to be safe, i.e. to not veto any signal candidate with $2 \mathcal{F}_{\mathrm{DM} \text {-on }}$ in the range of the candidates under consideration. In particular we find that for candidates with $2 \mathcal{F}_{\mathrm{DM}-\text { on }}<500$, after the third follow-up, $2 \mathcal{F}_{\mathrm{DM} \text {-off }}^{\mathrm{thr}}=62$. The threshold increases for candidates with $2 \mathcal{F}_{\text {DM-on }}>500$, scaling linearly with the candidates $2 \mathcal{F}_{\text {DM-on }}$ (see Fig. 4 of [36]).

As described in [36], the DM-off search is first run using data from both detectors and a search grid which is ten times coarser in $f$ and $\dot{f}$ than the stage 3 search. The coarser search grid is used to minimize computational cost. 653 of the 6349 candidates pass the $2 \mathcal{F}_{\mathrm{DM} \text {-off }}^{\text {thr }}$ threshold. These surviving candidates undergo another similar search, except that the search is performed separately on the data from each of the LIGO detectors. We search each detector separately because a detector artifact present only in one detector may still pass the previous, multidetector search, as its significance is "diluted" by the clean data of the other detector. 101 candidates survive, and undergo a final DMoff search stage. This search uses the fine grid parameters of the stage 3 search (Table II), covers the parameter space which resulted in the largest $2 \mathcal{F}_{\text {DM-off }}$ from the previous DM-off steps, and is performed three times, once using both detectors jointly and once for each of the two LIGO detectors. For a candidate to survive this stage it has to pass all three stage 3 searches.

Four candidates survive the full DM-off veto. Such veto is designed to be safe, i.e. not falsely dismiss real signals. However, its false alarm rate for noise disturbances is not fully characterized because very little is known about such weak and rare spectral disturbances, which this type of deep search unveils. This means that we cannot exclude that the four surviving candidates are in fact noise disturbances. The parameters of the candidates, after the third follow-up, are given in Table III. The $2 \mathcal{F}_{\text {DM-off }}$ values are also given in this table. 
TABLE III. Stage 3 follow-up results for each of the 4 candidates that survive the DM-off veto. For illustration purposes, in the 7 th and 8th column we show the values of the average single-detector detection statistics. Typically, for signals, the single-detector values do not exceed the multidetector $2 \overline{\mathcal{F}}$.

\begin{tabular}{|c|c|c|c|c|c|c|c|c|}
\hline ID & $f[\mathrm{~Hz}]$ & $\alpha[\mathrm{rad}]$ & $\delta[\mathrm{rad}]$ & $\dot{f}[\mathrm{~Hz} / \mathrm{s}]$ & $2 \overline{\mathcal{F}}$ & $2 \overline{\mathcal{F}}_{\mathrm{H} 1}$ & $2 \overline{\mathcal{F}}_{\mathrm{L} 1}$ & $2 \mathcal{F}_{\text {DM-off }}$ \\
\hline 1 & 58.970435900 & 1.87245 & -0.51971 & $-1.081102 \times 10^{-9}$ & 81.4 & 48.5 & 33.4 & 55 \\
\hline 2 & 62.081409292 & 4.98020 & 0.58542 & $-2.326246 \times 10^{-9}$ & 81.9 & 45.5 & 39.0 & 52 \\
\hline 3 & 97.197674733 & 5.88374 & -0.76773 & $2.28614 \times 10^{-10}$ & 86.5 & 55.0 & 31.8 & 58 \\
\hline 4 & 99.220728369 & 2.842702 & -0.469603 & $-2.498113 \times 10^{-9}$ & 80.2 & 41.4 & 45.8 & 55 \\
\hline
\end{tabular}

TABLE IV. Search parameters, rounded to the first decimal place, for the follow-up of surviving LIGO O1 candidates in LIGO O2 data. $T_{\text {ref }}$ is the reference time that defines the frequency and frequency derivative values.

\begin{tabular}{lc}
\hline \hline Parameter & Value \\
\hline$T_{\text {coh }}$ & $2160 \mathrm{hrs}$ \\
$T_{\text {ref }}$ & $1168447494.5 \mathrm{GPS} \mathrm{sec}$ \\
$N_{\text {seg }}$ & 1 \\
$\delta f$ & $9.0 \times 10^{-8} \mathrm{~Hz}$ \\
$\delta f_{c}$ & $1.1 \times 10^{-13} \mathrm{~Hz} / \mathrm{s}$ \\
$\gamma$ & 1 \\
$m_{\text {sky }}$ & $4 \times 10^{-7}$ \\
\hline \hline
\end{tabular}

\section{F. Follow-up in LIGO 02 data}

If the signal candidates surviving the $\mathrm{O} 1$ search are standard continuous wave signals, i.e. continuous wave signals arising from sources that radiate steadily over many years, they should be present in data from the Advanced LIGO's second observing run $(\mathrm{O} 2)$ with the same parameters. We perform a follow-up search using three months of O2 data, collected from November 30, 2016 to February 28, 2017.

The candidate parameters in Table III are translated to the $\mathrm{O} 2$ midtime, which is the reference time of the new search. The parameter space covered by the search is determined by the uncertainty on the candidate parameters in Eq. (5). The frequency region is widened to account for the spin-down uncertainty. The $\mathrm{O} 2$ follow-up covers a frequency range of $\pm 5.15 \times 10^{-4} \mathrm{~Hz}$ around the candidates.

The search parameters of the $\mathrm{O} 2$ follow-up are given in Table IV. The expected loudest $2 \overline{\mathcal{F}}$ per follow-up search due to Gaussian noise alone is $52 \pm 3$, assuming independent search templates.

If a candidate in Table III were due to a signal, the loudest $2 \overline{\mathcal{F}}$ expected after the follow-up would be the value given in the second column of Table V. This expected value is obtained by scaling the $2 \overline{\mathcal{F}}$ in Table III according to the different duration and the different noise levels between the data set used for the third follow-up and the $\mathrm{O} 2$ data set. The expected $2 \overline{\mathcal{F}}$ also folds in a conservative factor of 0.9 due to a different mismatch of the $\mathrm{O} 2$ template grid with
TABLE V. Highest $2 \overline{\mathcal{F}}$ expected after the follow-up in O2 data, if the candidates were due to a signal, compared with the highest $2 \overline{\mathcal{F}}$ recovered from the follow-up. The $2 \overline{\mathcal{F}}$ expected in Gaussian noise data is $52 \pm 3$.

\begin{tabular}{lcc}
\hline \hline Candidate & Expected $2 \overline{\mathcal{F}} \pm 1 \sigma$ & Loudest $2 \overline{\mathcal{F}}$ recovered \\
\hline 1 & $85 \pm 18$ & 44 \\
2 & $90 \pm 19$ & 52 \\
3 & $84 \pm 18$ & 49 \\
4 & $77 \pm 17$ & 47 \\
\hline \hline
\end{tabular}

respect to the template grid used for the third follow-up. Thus the expected $2 \overline{\mathcal{F}}$ in Table $\mathrm{V}$ is a conservative estimate for the minimum $2 \overline{\mathcal{F}}$ that we would expect from a signal candidate.

The loudest $2 \overline{\mathcal{F}}$ after the follow-up in $\mathrm{O} 2$ data is also given in Table $\mathrm{V}$. The loudest $2 \overline{\mathcal{F}}$ recovered for each candidate are $\approx 2 \sigma$ below the expected $2 \overline{\mathcal{F}}$ for a signal candidate. The recovered $2 \overline{\mathcal{F}}$ are consistent with what is expected from Gaussian data. We conclude that it is unlikely that any of the candidates in Table III arises from a long-lived astronomical source of continuous gravitational waves.

\section{RESULTS}

\section{A. Upper limits on the gravitational wave amplitude}

The search did not reveal any continuous gravitational wave signal in the parameter volume that was searched. We hence set frequentist $90 \%$ confidence upper limits on the maximum gravitational wave amplitude consistent with this null result in $0.5 \mathrm{~Hz}$ bands of the $\mathrm{O} 1$ data, $h_{0}^{90 \%}(f)$. Specifically, $h_{0}^{90 \%}(f)$ is the GW amplitude such that $90 \%$ of a population of signals with parameter values in our search range would have been detected by our search. We determined the upper limits in bands that were marked as undisturbed in Sec. III B. These upper limits may not hold for frequency bands that were marked as mildly disturbed, which we now consider disturbed as they were excluded by the analysis. These bands, as well as bands which were excluded from further analysis, are identified in Appendix A 3, Table VIII. 


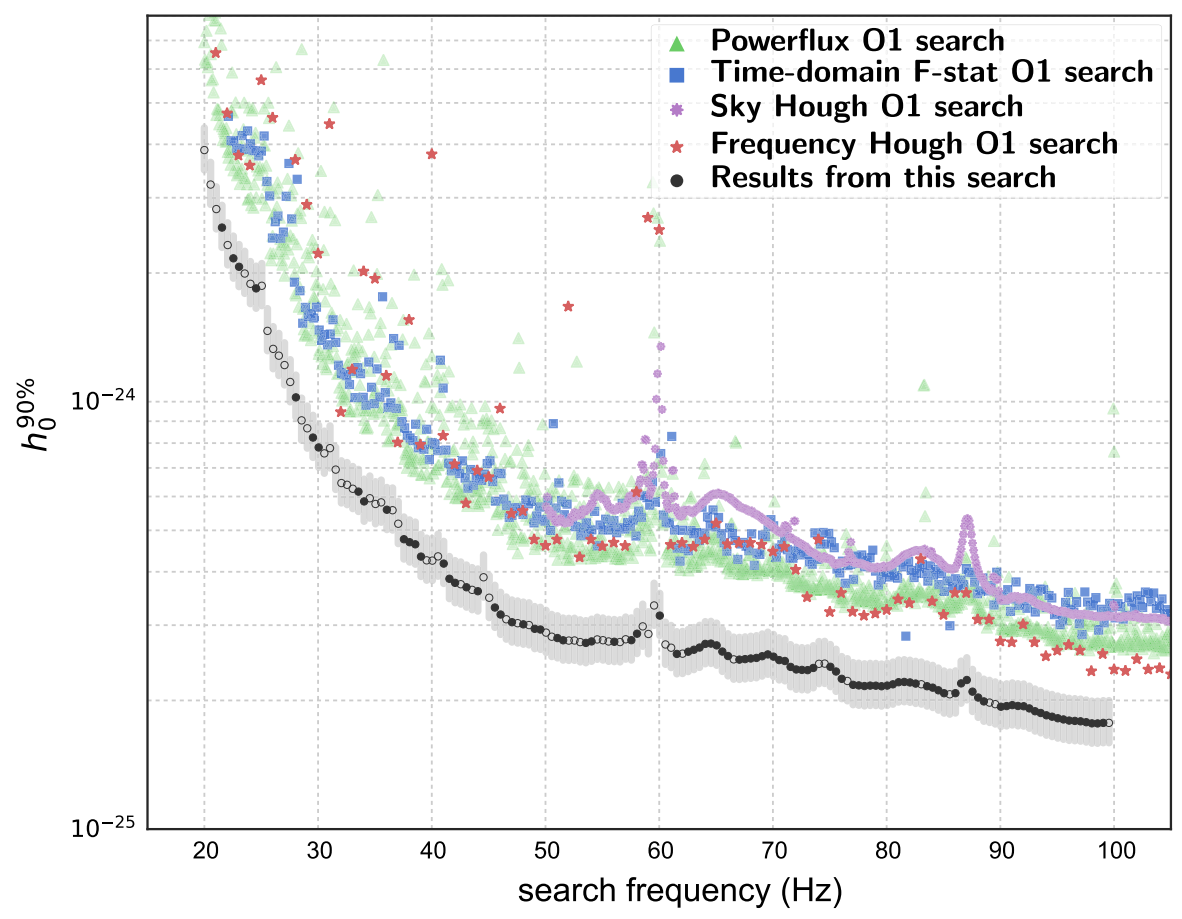

FIG. 10. $90 \%$ confidence upper limits on the gravitational wave amplitude of continuous gravitational wave signals with frequency in $0.5 \mathrm{~Hz}$ bands and with spin-down values within the searched range. The lowest set of points (black circles) are the results of this search. The empty circles denote half-Hz bands where the upper limit value does not hold for all frequencies in that interval. A list of the excluded frequencies is given in the Appendix. The lighter grey region around the upper limit points shows the $11 \%$ relative difference bracket between upper limits inferred with the procedure described in Sec. V and upper limits that would have been derived (at great computational expense) with direct measurements in all half-Hz bands. We estimate that less than $\sim 0.5 \%$ of the upper limit points would fall outside of this bracket if they were derived with the direct-measurement method in Gaussian noise. For comparison we also plot the most recent upper limits results in this frequency range from $\mathrm{O} 1$ data obtained with various search pipelines [38]. The better sensitivity of this search is due to the long coherent observation time used. We note also that the searches of [38] cover a broader frequency and spin-down range than the search presented here. All upper limits presented here are population-averaged limits over the full sky and source polarization.

Since an actual full-scale fake-signal injection-andrecovery Monte Carlo for the entire set of follow-ups in every $0.5 \mathrm{~Hz}$ band is prohibitive, in the same spirit as $[2,5,31]$, we perform such a study in a limited set of trial bands. We choose 20 half- $\mathrm{Hz}$ bands to measure the upper limits. If these half- $\mathrm{Hz}$ bands include $50 \mathrm{mHz}$ bands which were not marked undisturbed, no upper limit injections are made in those $50 \mathrm{mHz}$ bands.

The amplitudes of the fake signals bracket the $90 \%$ confidence region typically between $70 \%$ and $100 \%$. The $h_{0}$ versus confidence data is fit in this region with a sigmoid of the form

$$
C\left(h_{0}\right)=\frac{1}{1+\exp \left(\frac{\mathrm{a}-h_{0}}{\mathrm{~b}}\right)}
$$

and the $h_{0}^{90 \%}$ value is read off of this curve. The fitting procedure $^{3}$ yields the best-fit $a$ and $b$ values and the covariance matrix. Given the binomial confidence values uncertainties,

\footnotetext{
${ }^{3}$ We used the linfit Matlab routine.
}

using the covariance matrix we estimate the $h_{0}^{90 \%}$ uncertainty.

For each of these frequency bands we determine the sensitivity depth $\mathcal{D}^{90 \%}$ [37] of the search corresponding to $h_{0}^{90 \%}(f)$ :

$$
\mathcal{D}^{90 \%}:=\frac{\sqrt{S_{h}(f)}}{h_{0}^{90 \%}(f)}[1 / \sqrt{\mathrm{Hz}}],
$$

where $\sqrt{S_{h}(f)}$ is the noise level of the data as a function of frequency.

As representative of the sensitivity depth of this hierarchical search, we take the average of the measured depths at different frequencies: $48.7[1 / \sqrt{\mathrm{Hz}}]$. We then determine the $90 \%$ upper limits by substituting this value in Eq. (8) for $\mathcal{D}^{90 \%}$.

The upper limit that we get with this procedure, in general, yields a different number compared to the upper limit directly measured as done in the 20 test bands. An $11 \%$ relative error bracket comprises the range of variation observed on the measured sensitivity depths, including the 
uncertainties on the single measurements. So we take this as a generous estimate of the range of variability of the upper limit values introduced by the estimation procedure. If the data were Gaussian this bracket would yield a $\sim 0.5 \%$ probability of a measured upper limit falling outside of this bracket.

As a sanity check we measure the upper limits in five half-Hz bands which were not used to determine the average sensitivity depth. In each case we find that the measured sensitivity depth falls well within $11 \%$ of the average: $48.7[1 / \sqrt{\mathrm{Hz}}]$.

Figure 10 shows the upper limits as a function of frequency. They are also presented in tabular form in the Appendix with the uncertainties indicating the range of variability introduced by the estimation procedure. The associated uncertainties amount to $220 \%$ when also including $10 \%$ amplitude calibration uncertainty. The most constraining upper limit in the band $98.5-99 \mathrm{~Hz}$, close to the highest frequency, where the detector is most sensitive, is $1.8 \times 10^{-25}$. At the lowest end of the frequency range, at $20 \mathrm{~Hz}$, the upper limit rises to $3.9 \times 10^{-24}$.

\section{B. Upper limits on the source ellipticity}

In general not all the rotational kinetic energy lost is due to GW emission. Following [39], we define $x$ to be the fraction of the spin-down rotational energy emitted in gravitational waves. The star's ellipticity necessary to sustain such emission is

$$
\varepsilon(f, x \dot{f})=\sqrt{\frac{5 c^{5}}{32 \pi^{4} G} \frac{x|\dot{f}|}{I f^{5}}},
$$

where $c$ is the speed of light, $G$ is the gravitational constant, $f$ is the GW frequency, and $I$ is the principal moment of inertia of the star. Correspondingly, $x \dot{f}$ is the spin-down rate that accounts for the emission of GWs and this is why we refer to it as the GW spin-down. The gravitational wave amplitude $h_{0}$ at the detector coming from a GW source at a distance $D$ from Earth is

$$
h_{0}(f, D)=\frac{4 G I \pi^{2}}{c^{4}} \frac{\varepsilon f^{2}}{D} .
$$

Based on this last equation, we can use the GW amplitude upper limits to bound the minimum distance for compact objects emitting continuous gravitational waves under different assumptions on the ellipticity of the objects. This is shown in Fig. 11. Above $55 \mathrm{~Hz}$ we can exclude sources with ellipticities larger than $10^{-5}$ (corresponding to $x$ values larger than $3.2 \times 10^{-5} \frac{2.6 \times 10^{-9} \mathrm{~Hz} / \mathrm{s}}{|\dot{f}|}$ ) within $100 \mathrm{pc}$ of Earth. Rough estimates are that there should be of order $10^{4}$ neutron stars within this volume.

The dashed line in Fig. 11 is the spin-down ellipticity $[x=1$ in Eq. (9)] for an object spinning down at half the

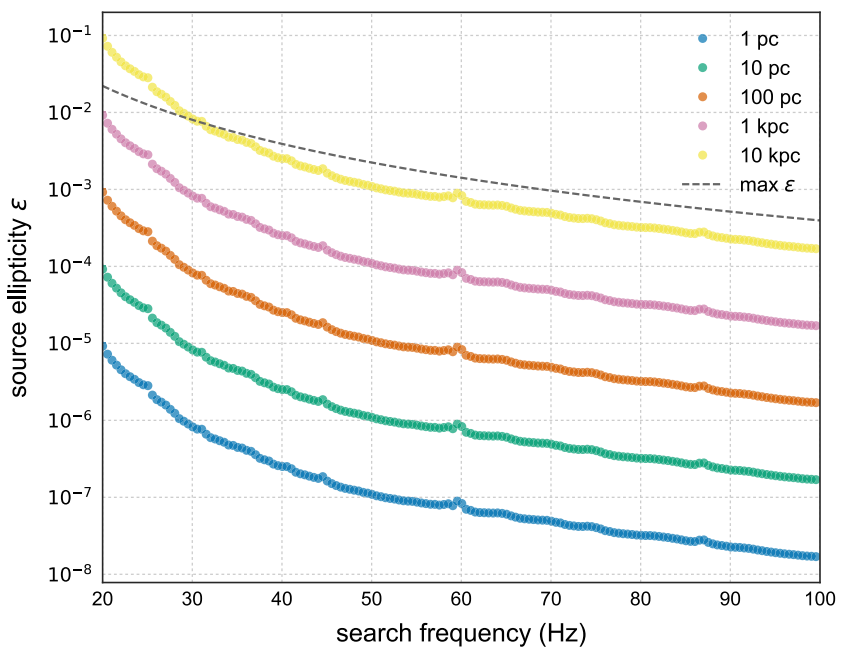

FIG. 11. Ellipticity $\varepsilon$ of a source at a distance $D$ emitting continuous gravitational waves that would have been detected by this search, assuming a source $\dot{f}$ within the range covered by this search. The dashed line shows the spin-down ellipticity for the highest magnitude spin-down parameter value searched, $2.6 \times 10^{-9} \mathrm{~Hz} / \mathrm{s}$. The spin-down ellipticity is the ellipticity necessary for all the lost rotational kinetic energy to be emitted in gravitational waves. If we assume that the observed spin-down is all actual spin-down of the object, then no ellipticities could be possible above the dashed curve. In reality the observed and actual spin-down could differ due to radial acceleration of the source. In this case the actual spin-down of the object may even be larger than the apparent one. In this case our search would be sensitive to objects with ellipticities above the dashed line.

maximum searched gravitational wave signal spin-down value $\left(|\dot{f}|=2.6 \times 10^{-9} \mathrm{~Hz} / \mathrm{s}\right)$. This is the maximum ellipticity that this search probes; we can make no claim on sources with ellipticities greater than this. For a normal neutron star one might expect the majority of the spin-down to be due to non gravitational-wave emission. If, for instance, one believes that only $1 \%$ of the spin-down is due to gravitational wave emission $(x=0.01)$ then, from Eq. (9), the maximum ellipticity needs to be divided by ten and the dashed line in Fig. 11 drops by the same factor.

The results in Fig. 11 assume a fiducial value of the principal moment of inertia of $10^{38} \mathrm{~kg} \mathrm{~m}^{2}$. The upper limits can be scaled to any assumption for $I$ using Eq. (10).

\section{CONCLUSIONS}

This search concentrates the computing power of Einstein@Home in a relatively small frequency range at low frequencies where all-sky searches are significantly "cheaper" than at higher frequencies. For this reason, the initial search could be set up with a very long coherent observation time of $210 \mathrm{hr}$ and this yields a record sensitivity depth of $48.7[1 / \sqrt{\mathrm{Hz}}]$.

The $\mathrm{O} 1$ data set in the low frequency range investigated with this search is significantly more polluted by coherent 
spectral artifacts than most of the data sets from the InitialLIGO science runs. Because of this, even a relatively high threshold on the detection statistic of the first search yields tens of thousands of candidates, rather than just $O(100)$. We follow each of them up through a hierarchy of three further stages at the end of which $O(7000)$ survive. After the application of a newly developed Doppler-modulationoff veto, four survive.

Due to the presence of coherent spectral artifacts, no assumption can be made about the noise background of the search. On the other hand, we cannot run an Einstein@Home search many times to estimate such background. ${ }^{4}$ Because of this, measuring the significance of the four candidates at the end of the third follow-up stage is not trivial. However, the parameter uncertainty around the four candidates after the third follow-up stage is small [Eq. (6)], so with an independent data set we can verify the findings of the third stage and estimate its background. This means that we could assign a measure of confidence to any candidate that might survive the search on the new data set.

The four candidates which survive the Dopplermodulation-off veto are followed up with a fully coherent search using three months of $\mathrm{O} 2$ data, which produces results completely consistent with Gaussian noise and falls short of the predictions under the signal hypothesis. We hence proceed to set upper limits on the intrinsic GW amplitude $h_{0}$. The hierarchical follow-up procedure presented here has also been used to follow-up outliers from other all-sky searches in O1 data with various search pipelines [38].

The smallest value of the GW amplitude upper limit is $1.8 \times 10^{-25}$ in the band $98.5-99 \mathrm{~Hz}$. Figure 10 shows the upper limit values as a function of search frequency. Our upper limits are the tightest ever placed for this population of signals, and are a factor 1.5-2 smaller than the most recent upper limits [38]. We note that [38] presents results from four different all-sky search pipelines covering a broader frequency and spin-down range than the one explored here. The coherent time baseline for all these pipelines is significantly shorter than the $210 \mathrm{hr}$ used by the very first stage of this search. This limits the sensitivity of those searches but it makes them more robust to deviations in the signal waveform from the target waveform, compared to this search. We finally note that because the data is plagued in this low frequency region by coherent disturbances, the two procedures $[34,36]$ are essential to reach the final sensitivity: without them a much higher detection threshold would have been needed, ultimately resulting in a degraded sensitivity/astrophysical reach.

Translating the upper limits on the GW amplitude to upper limits on the ellipticity of the GW source, we find that for frequencies above $55 \mathrm{~Hz}$ our results exclude isolated compact objects with spin-down ellipticities of

\footnotetext{
${ }^{4}$ By producing many (off-source) realizations of the search results.
}

$10^{-5} \sqrt{10^{38} \mathrm{~kg} \mathrm{~m}^{2} / \mathrm{I}}$ (corresponding to GW spin-downs between $10^{-14} \mathrm{~Hz} / \mathrm{s}$ and $10^{-13} \mathrm{~Hz} / \mathrm{s}$ ) or higher, within $100 \mathrm{pc}$ of Earth. For the population of known pulsars we know that the spin-down ellipticity is generally an overestimate of the actual ellipticity. However, for other objects, belonging to the population of possible continuous wave emitters that we do not see, this might not be the case. So we present the bounds on our results (the dashed line in Fig. 11) in terms of the spin-down ellipticity and leave it to the reader to derive the reach for the ellipticity value that best represents the class of sources in which they are interested.

\section{ACKNOWLEDGMENTS}

The authors gratefully acknowledge the support of the United States National Science Foundation (NSF) for the construction and operation of the LIGO Laboratory and Advanced LIGO as well as the Science and Technology Facilities Council (STFC) of the United Kingdom, the MaxPlanck-Society (MPS), and the State of Niedersachsen/ Germany for support of the construction of Advanced LIGO and construction and operation of the GEO600 detector. Additional support for Advanced LIGO was provided by the Australian Research Council. The authors gratefully acknowledge the Italian Istituto Nazionale di Fisica Nucleare (INFN), the French Centre National de la Recherche Scientifique (CNRS) and the Foundation for Fundamental Research on Matter supported by the Netherlands Organisation for Scientific Research, for the construction and operation of the Virgo detector and the creation and support of the EGO consortium. The authors also gratefully acknowledge research support from these agencies as well as by the Council of Scientific and Industrial Research of India, Department of Science and Technology, India, Science \& Engineering Research Board (SERB), India, Ministry of Human Resource Development, India, the Spanish Ministerio de Economía y Competitividad, the Vicepresidència i Conselleria d'Innovació, Recerca i Turisme and the Conselleria d'Educació i Universitat del Govern de les Illes Balears, the National Science Centre of Poland, the European Commission, the Royal Society, the Scottish Funding Council, the Scottish Universities Physics Alliance, the Hungarian Scientific Research Fund (OTKA), the Lyon Institute of Origins (LIO), the National Research Foundation of Korea, Industry Canada and the Province of Ontario through the Ministry of Economic Development and Innovation, the Natural Science and Engineering Research Council Canada, Canadian Institute for Advanced Research, the Brazilian Ministry of Science, Technology, and Innovation, International Center for Theoretical Physics South American Institute for Fundamental Research (ICTP-SAIFR), Russian Foundation for Basic Research, the Leverhulme Trust, the Research Corporation, Ministry of Science and Technology (MOST), Taiwan and the Kavli Foundation. The authors gratefully acknowledge the support 
of the NSF, STFC, MPS, INFN, CNRS and the State of Niedersachsen/Germany for provision of computational resources. The authors also gratefully acknowledge the support of the many thousands of Einstein@Home volunteers, without whom this search would not have been possible.

This document has been assigned LIGO Laboratory document No. LIGO-P1700127.

\section{APPENDIX: TABULAR DATA}

See Table VI.

\section{Upper limit values}

TABLE VI. First frequency of each half-Hz signal frequency band in which we set upper limits and upper limit value for that band. The uncertainties correspond to the $11 \%$ relative difference bracket discussed in Sec. V.

\begin{tabular}{|c|c|c|c|c|c|c|c|}
\hline$f(\mathrm{~Hz})$ & $h_{0}^{90 \%} \times 10^{25}$ & $f(\mathrm{~Hz})$ & $h_{0}^{90 \%} \times 10^{25}$ & $f(\mathrm{~Hz})$ & $h_{0}^{90 \%} \times 10^{25}$ & $f(\mathrm{~Hz})$ & $h_{0}^{90 \%} \times 10^{25}$ \\
\hline 20.00 & $38.8 \pm 4.9$ & 20.55 & $32.2 \pm 4.1$ & 21.05 & $28.2 \pm 3.6$ & 21.55 & $25.5 \pm 3.3$ \\
\hline 22.05 & $23.3 \pm 3.0$ & 22.55 & $21.6 \pm 2.8$ & 23.05 & $20.7 \pm 2.6$ & 23.55 & $19.9 \pm 2.5$ \\
\hline 24.05 & $18.9 \pm 2.4$ & 24.55 & $18.4 \pm 2.3$ & 25.05 & $18.7 \pm 2.4$ & 25.55 & $14.6 \pm 1.9$ \\
\hline 26.05 & $13.3 \pm 1.7$ & 26.55 & $12.8 \pm 1.6$ & 27.05 & $12.2 \pm 1.6$ & 27.55 & $11.1 \pm 1.4$ \\
\hline 28.05 & $10.2 \pm 1.3$ & 28.55 & $9.0 \pm 1.2$ & 29.05 & $8.7 \pm 1.1$ & 29.55 & $8.2 \pm 1.0$ \\
\hline 30.05 & $7.8 \pm 1.0$ & 30.55 & $7.6 \pm 1.0$ & 31.05 & $7.8 \pm 1.0$ & 31.55 & $6.9 \pm 0.9$ \\
\hline 32.05 & $6.5 \pm 0.8$ & 32.55 & $6.4 \pm 0.8$ & 33.05 & $6.3 \pm 0.8$ & 33.55 & $6.2 \pm 0.8$ \\
\hline 34.05 & $5.8 \pm 0.7$ & 34.55 & $5.9 \pm 0.8$ & 35.05 & $5.8 \pm 0.7$ & 35.55 & $5.8 \pm 0.7$ \\
\hline 36.05 & $5.6 \pm 0.7$ & 36.55 & $5.6 \pm 0.7$ & 37.05 & $5.2 \pm 0.7$ & 37.55 & $4.8 \pm 0.6$ \\
\hline 38.05 & $4.7 \pm 0.6$ & 38.55 & $4.6 \pm 0.6$ & 39.05 & $4.3 \pm 0.6$ & 39.55 & $4.3 \pm 0.5$ \\
\hline 40.05 & $4.2 \pm 0.5$ & 40.55 & $4.3 \pm 0.6$ & 41.05 & $4.2 \pm 0.5$ & 41.55 & $3.9 \pm 0.5$ \\
\hline 42.05 & $3.8 \pm 0.5$ & 42.55 & $3.7 \pm 0.5$ & 43.05 & $3.7 \pm 0.5$ & 43.55 & $3.6 \pm 0.5$ \\
\hline 44.05 & $3.6 \pm 0.5$ & 44.55 & $3.9 \pm 0.5$ & 45.05 & $3.5 \pm 0.4$ & 45.55 & $3.3 \pm 0.4$ \\
\hline 46.05 & $3.2 \pm 0.4$ & 46.55 & $3.1 \pm 0.4$ & 47.05 & $3.0 \pm 0.4$ & 47.55 & $3.0 \pm 0.4$ \\
\hline 48.05 & $3.0 \pm 0.4$ & 48.55 & $3.0 \pm 0.4$ & 49.05 & $2.9 \pm 0.4$ & 49.55 & $2.9 \pm 0.4$ \\
\hline 50.05 & $2.9 \pm 0.4$ & 50.55 & $2.8 \pm 0.4$ & 51.05 & $2.8 \pm 0.4$ & 51.55 & $2.8 \pm 0.4$ \\
\hline 52.05 & $2.8 \pm 0.4$ & 52.55 & $2.8 \pm 0.4$ & 53.05 & $2.7 \pm 0.3$ & 53.55 & $2.7 \pm 0.3$ \\
\hline 54.05 & $2.7 \pm 0.3$ & 54.55 & $2.8 \pm 0.4$ & 55.05 & $2.8 \pm 0.4$ & 55.55 & $2.7 \pm 0.3$ \\
\hline 56.05 & $2.7 \pm 0.3$ & 56.55 & $2.7 \pm 0.3$ & 57.05 & $2.8 \pm 0.4$ & 57.55 & $2.8 \pm 0.4$ \\
\hline 58.05 & $2.9 \pm 0.4$ & 58.55 & $3.0 \pm 0.4$ & 59.05 & $2.9 \pm 0.4$ & 59.55 & $3.3 \pm 0.4$ \\
\hline 60.05 & $3.2 \pm 0.4$ & 60.55 & $2.7 \pm 0.3$ & 61.05 & $2.7 \pm 0.3$ & 61.55 & $2.6 \pm 0.3$ \\
\hline 62.05 & $2.6 \pm 0.3$ & 62.55 & $2.6 \pm 0.3$ & 63.05 & $2.6 \pm 0.3$ & 63.55 & $2.7 \pm 0.3$ \\
\hline 64.05 & $2.7 \pm 0.3$ & 64.55 & $2.7 \pm 0.3$ & 65.05 & $2.7 \pm 0.3$ & 65.55 & $2.6 \pm 0.3$ \\
\hline 66.05 & $2.5 \pm 0.3$ & 66.55 & $2.5 \pm 0.3$ & 67.05 & $2.5 \pm 0.3$ & 67.55 & $2.5 \pm 0.3$ \\
\hline 68.05 & $2.5 \pm 0.3$ & 68.55 & $2.5 \pm 0.3$ & 69.05 & $2.5 \pm 0.3$ & 69.55 & $2.6 \pm 0.3$ \\
\hline 70.05 & $2.5 \pm 0.3$ & 70.55 & $2.5 \pm 0.3$ & 71.05 & $2.5 \pm 0.3$ & 71.55 & $2.4 \pm 0.3$ \\
\hline 72.05 & $2.4 \pm 0.3$ & 72.55 & $2.4 \pm 0.3$ & 73.05 & $2.4 \pm 0.3$ & 73.55 & $2.4 \pm 0.3$ \\
\hline 74.05 & $2.4 \pm 0.3$ & 74.55 & $2.4 \pm 0.3$ & 75.05 & $2.4 \pm 0.3$ & 75.55 & $2.3 \pm 0.3$ \\
\hline 76.05 & $2.2 \pm 0.3$ & 76.55 & $2.2 \pm 0.3$ & 77.05 & $2.2 \pm 0.3$ & 77.55 & $2.2 \pm 0.3$ \\
\hline 78.05 & $2.2 \pm 0.3$ & 78.55 & $2.2 \pm 0.3$ & 79.05 & $2.2 \pm 0.3$ & 79.55 & $2.2 \pm 0.3$ \\
\hline 80.05 & $2.2 \pm 0.3$ & 80.55 & $2.2 \pm 0.3$ & 81.05 & $2.2 \pm 0.3$ & 81.55 & $2.2 \pm 0.3$ \\
\hline 82.05 & $2.2 \pm 0.3$ & 82.55 & $2.2 \pm 0.3$ & 83.05 & $2.2 \pm 0.3$ & 83.55 & $2.2 \pm 0.3$ \\
\hline 84.05 & $2.1 \pm 0.3$ & 84.55 & $2.1 \pm 0.3$ & 85.05 & $2.1 \pm 0.3$ & 85.55 & $2.1 \pm 0.3$ \\
\hline 86.05 & $2.1 \pm 0.3$ & 86.55 & $2.2 \pm 0.3$ & 87.05 & $2.2 \pm 0.3$ & 87.55 & $2.1 \pm 0.3$ \\
\hline 88.05 & $2.0 \pm 0.3$ & 88.55 & $2.0 \pm 0.3$ & 89.05 & $2.0 \pm 0.3$ & 89.55 & $2.0 \pm 0.2$ \\
\hline 90.05 & $1.9 \pm 0.2$ & 90.55 & $1.9 \pm 0.2$ & 91.05 & $2.0 \pm 0.2$ & 91.55 & $1.9 \pm 0.2$ \\
\hline 92.05 & $1.9 \pm 0.2$ & 92.55 & $1.9 \pm 0.2$ & 93.05 & $1.9 \pm 0.2$ & 93.55 & $1.9 \pm 0.2$ \\
\hline 94.05 & $1.8 \pm 0.2$ & 94.55 & $1.8 \pm 0.2$ & 95.05 & $1.8 \pm 0.2$ & 95.55 & $1.8 \pm 0.2$ \\
\hline 96.05 & $1.8 \pm 0.2$ & 96.55 & $1.8 \pm 0.2$ & 97.05 & $1.8 \pm 0.2$ & 97.55 & $1.8 \pm 0.2$ \\
\hline 98.05 & $1.8 \pm 0.2$ & 98.55 & $1.8 \pm 0.2$ & 99.05 & $1.8 \pm 0.2$ & 99.55 & $1.8 \pm 0.2$ \\
\hline
\end{tabular}




\section{Cleaned-out frequency bins}

See Table VII

TABLE VII. Instrumental lines identified and cleaned before the Einstein@Home runs. The different columns represent (I) the central frequency of the instrumental line; (II) low-frequency side (LFS) of the knockout band; (III) high-frequency side (HFS) of the knockout band; (IV) the interferometer in which the instrumental lines were identified.

\begin{tabular}{|c|c|c|c|}
\hline$f_{\mathrm{L}}(\mathrm{Hz})$ & LFS (Hz) & HFS $(\mathrm{Hz})$ & IFO \\
\hline 19.9995 & 0.001 & 0.001 & $\mathrm{~L}$ \\
\hline 20.0 & 0.001 & 0.001 & $\mathrm{H}$ \\
\hline 20.24999 & 0.001 & 0.001 & $\mathrm{H}$ \\
\hline 20.25014 & 0.001 & 0.001 & $\mathrm{~L}$ \\
\hline 20.5 & 0.001 & 0.001 & $\mathrm{H}$ \\
\hline 20.5 & 0.001 & 0.001 & $\mathrm{~L}$ \\
\hline 20.7163 & 0.002 & 0.002 & $\mathrm{~L}$ \\
\hline 20.73 & 0.002 & 0.002 & $\mathrm{~L}$ \\
\hline 20.74121875 & 0.001 & 0.001 & $\mathrm{H}$ \\
\hline 20.7423125 & 0.001 & 0.001 & $\mathrm{H}$ \\
\hline 20.9995 & 0.001 & 0.001 & $\mathrm{~L}$ \\
\hline 21.0 & 0.001 & 0.001 & $\mathrm{H}$ \\
\hline 21.24998 & 0.001 & 0.001 & $\mathrm{H}$ \\
\hline 21.25011 & 0.001 & 0.001 & $\mathrm{~L}$ \\
\hline 21.3575 & 0.001 & 0.001 & $\mathrm{~L}$ \\
\hline 21.3842 & 0.001 & 0.001 & $\mathrm{~L}$ \\
\hline 21.41043 & 0.001 & 0.001 & $\mathrm{~L}$ \\
\hline 21.41043 & 0.001 & 0.001 & $\mathrm{~L}$ \\
\hline 21.4374 & 0.001 & 0.001 & $\mathrm{~L}$ \\
\hline 21.4639 & 0.001 & 0.001 & $\mathrm{~L}$ \\
\hline 21.499987 & 0.001 & 0.001 & $\mathrm{~L}$ \\
\hline 21.5 & 0.001 & 0.001 & $\mathrm{H}$ \\
\hline 21.7028 & 0.002 & 0.002 & $\mathrm{~L}$ \\
\hline 21.7165 & 0.002 & 0.002 & $\mathrm{~L}$ \\
\hline 21.7344 & 0.001 & 0.001 & $\mathrm{~L}$ \\
\hline 21.9995 & 0.001 & 0.001 & $\mathrm{~L}$ \\
\hline 22.0 & 0.001 & 0.001 & $\mathrm{H}$ \\
\hline 22.24997 & 0.001 & 0.001 & $\mathrm{H}$ \\
\hline 22.25008 & 0.001 & 0.001 & $\mathrm{~L}$ \\
\hline 22.499974 & 0.001 & 0.001 & $\mathrm{~L}$ \\
\hline 22.5 & 0.001 & 0.001 & $\mathrm{H}$ \\
\hline 22.6893 & 0.002 & 0.002 & $\mathrm{~L}$ \\
\hline 22.7 & 0.0005 & 0.0005 & $\mathrm{~L}$ \\
\hline 22.703 & 0.002 & 0.002 & $\mathrm{~L}$ \\
\hline 22.72233 & 0.001 & 0.001 & $\mathrm{~L}$ \\
\hline 22.815340625 & 0.001 & 0.001 & $\mathrm{H}$ \\
\hline 22.81654375 & 0.001 & 0.001 & $\mathrm{H}$ \\
\hline 22.9995 & 0.001 & 0.001 & $\mathrm{~L}$ \\
\hline 23.0 & 0.001 & 0.001 & $\mathrm{H}$ \\
\hline 23.24996 & 0.001 & 0.001 & $\mathrm{H}$ \\
\hline 23.25005 & 0.001 & 0.001 & $\mathrm{~L}$ \\
\hline 23.3039 & 0.001 & 0.001 & $\mathrm{~L}$ \\
\hline 23.3306 & 0.001 & 0.001 & $\mathrm{~L}$ \\
\hline 23.35683 & 0.001 & 0.001 & $\mathrm{~L}$ \\
\hline
\end{tabular}

TABLE VII. (Continued)

\begin{tabular}{|c|c|c|c|}
\hline$f_{\mathrm{L}}(\mathrm{Hz})$ & LFS $(\mathrm{Hz})$ & HFS $(\mathrm{Hz})$ & IFO \\
\hline 23.35683 & 0.001 & 0.001 & $\mathrm{~L}$ \\
\hline 23.3838 & 0.001 & 0.001 & $\mathrm{~L}$ \\
\hline 23.4103 & 0.001 & 0.001 & $\mathrm{~L}$ \\
\hline 23.499961 & 0.001 & 0.001 & $\mathrm{~L}$ \\
\hline 23.5 & 0.001 & 0.001 & $\mathrm{H}$ \\
\hline 23.6758 & 0.002 & 0.002 & $\mathrm{~L}$ \\
\hline 23.6895 & 0.002 & 0.002 & $\mathrm{~L}$ \\
\hline 23.71026 & 0.001 & 0.001 & $\mathrm{~L}$ \\
\hline 23.97079 & 0.0016 & 0.0008 & $\mathrm{~L}$ \\
\hline 23.9995 & 0.001 & 0.001 & $\mathrm{~L}$ \\
\hline 24.0 & 0.0005 & 0.0005 & $\mathrm{H}$ \\
\hline 24.0 & 0.001 & 0.001 & $\mathrm{H}$ \\
\hline 24.24995 & 0.001 & 0.001 & $\mathrm{H}$ \\
\hline 24.25002 & 0.001 & 0.001 & $\mathrm{~L}$ \\
\hline 24.499948 & 0.001 & 0.001 & $\mathrm{~L}$ \\
\hline 24.5 & 0.001 & 0.001 & $\mathrm{H}$ \\
\hline 24.6623 & 0.002 & 0.002 & $\mathrm{~L}$ \\
\hline 24.676 & 0.002 & 0.002 & $\mathrm{~L}$ \\
\hline 24.69819 & 0.001 & 0.001 & $\mathrm{~L}$ \\
\hline 24.8894625 & 0.001 & 0.001 & $\mathrm{H}$ \\
\hline 24.890775 & 0.001 & 0.001 & $\mathrm{H}$ \\
\hline 24.9995 & 0.001 & 0.001 & $\mathrm{~L}$ \\
\hline 25.0 & 0.001 & 0.001 & $\mathrm{H}$ \\
\hline 25.24994 & 0.001 & 0.001 & $\mathrm{H}$ \\
\hline 25.24999 & 0.001 & 0.001 & $\mathrm{~L}$ \\
\hline 25.2503 & 0.001 & 0.001 & $\mathrm{~L}$ \\
\hline 25.277 & 0.001 & 0.001 & $\mathrm{~L}$ \\
\hline 25.30323 & 0.001 & 0.001 & $\mathrm{~L}$ \\
\hline 25.30323 & 0.001 & 0.001 & $\mathrm{~L}$ \\
\hline 25.3302 & 0.001 & 0.001 & $\mathrm{~L}$ \\
\hline 25.3567 & 0.001 & 0.001 & $\mathrm{~L}$ \\
\hline 25.499935 & 0.001 & 0.001 & $\mathrm{~L}$ \\
\hline 25.5 & 0.001 & 0.001 & $\mathrm{H}$ \\
\hline 25.6 & 0.0005 & 0.0005 & $\mathrm{~L}$ \\
\hline 25.6488 & 0.002 & 0.002 & $\mathrm{~L}$ \\
\hline 25.6625 & 0.002 & 0.002 & $\mathrm{~L}$ \\
\hline 25.68612 & 0.001 & 0.001 & $\mathrm{~L}$ \\
\hline 25.9995 & 0.001 & 0.001 & $\mathrm{~L}$ \\
\hline 26.0 & 0.001 & 0.001 & $\mathrm{H}$ \\
\hline 26.24993 & 0.001 & 0.001 & $\mathrm{H}$ \\
\hline 26.24996 & 0.001 & 0.001 & $\mathrm{~L}$ \\
\hline 26.499922 & 0.001 & 0.001 & $\mathrm{~L}$ \\
\hline 26.5 & 0.001 & 0.001 & $\mathrm{H}$ \\
\hline 26.6353 & 0.002 & 0.002 & $\mathrm{~L}$ \\
\hline 26.649 & 0.002 & 0.002 & $\mathrm{~L}$ \\
\hline 26.67405 & 0.001 & 0.001 & $\mathrm{~L}$ \\
\hline 26.963584375 & 0.001 & 0.001 & $\mathrm{H}$ \\
\hline 26.96500625 & 0.001 & 0.001 & $\mathrm{H}$ \\
\hline 26.9995 & 0.001 & 0.001 & $\mathrm{~L}$ \\
\hline 27.0 & 0.001 & 0.001 & $\mathrm{H}$ \\
\hline 27.1967 & 0.001 & 0.001 & $\mathrm{~L}$ \\
\hline 27.2234 & 0.001 & 0.001 & $\mathrm{~L}$ \\
\hline 27.24963 & 0.001 & 0.001 & $\mathrm{~L}$ \\
\hline 27.24963 & 0.001 & 0.001 & $\mathrm{~L}$ \\
\hline 27.24992 & 0.001 & 0.001 & $\mathrm{H}$ \\
\hline
\end{tabular}


TABLE VII. (Continued)

\begin{tabular}{|c|c|c|c|c|c|c|c|}
\hline$\underline{f_{\mathrm{L}}(\mathrm{Hz})}$ & LFS $(\mathrm{Hz})$ & HFS (Hz) & IFO & $\underline{f_{\mathrm{L}}(\mathrm{Hz})}$ & LFS $(\mathrm{Hz})$ & HFS (Hz) & IFO \\
\hline 27.24993 & 0.001 & 0.001 & $\mathrm{~L}$ & 31.14243 & 0.001 & 0.001 & $\mathrm{~L}$ \\
\hline 27.2766 & 0.001 & 0.001 & $\mathrm{~L}$ & 31.1694 & 0.001 & 0.001 & $\mathrm{~L}$ \\
\hline 27.3031 & 0.001 & 0.001 & $\mathrm{~L}$ & 31.1959 & 0.001 & 0.001 & $\mathrm{~L}$ \\
\hline 27.499909 & 0.001 & 0.001 & $\mathrm{~L}$ & 31.2231 & 0.001 & 0.001 & $\mathrm{~L}$ \\
\hline 27.5 & 0.001 & 0.001 & $\mathrm{H}$ & 31.2495 & 0.001 & 0.001 & $\mathrm{~L}$ \\
\hline 27.6218 & 0.002 & 0.002 & $\mathrm{~L}$ & 31.24981 & 0.001 & 0.001 & $\mathrm{~L}$ \\
\hline 27.6355 & 0.002 & 0.002 & $\mathrm{~L}$ & 31.24988 & 0.001 & 0.001 & $\mathrm{H}$ \\
\hline 27.66198 & 0.001 & 0.001 & $\mathrm{~L}$ & 31.4 & 0.0005 & 0.0005 & $\mathrm{~L}$ \\
\hline 27.9995 & 0.001 & 0.001 & $\mathrm{~L}$ & 31.4127 & 0.003 & 0.003 & $\mathrm{H}$ \\
\hline 28.0 & 0.001 & 0.001 & $\mathrm{H}$ & 31.4149 & 0.003 & 0.003 & $\mathrm{H}$ \\
\hline 28.2499 & 0.001 & 0.001 & $\mathrm{~L}$ & 31.499857 & 0.001 & 0.001 & $\mathrm{~L}$ \\
\hline 28.24991 & 0.001 & 0.001 & $\mathrm{H}$ & 31.5 & 0.001 & 0.001 & $\mathrm{H}$ \\
\hline 28.499896 & 0.001 & 0.001 & $\mathrm{~L}$ & 31.5678 & 0.002 & 0.002 & $\mathrm{~L}$ \\
\hline 28.5 & 0.001 & 0.001 & $\mathrm{H}$ & 31.5815 & 0.002 & 0.002 & $\mathrm{~L}$ \\
\hline 28.5 & 0.0005 & 0.0005 & $\mathrm{~L}$ & 31.6137 & 0.001 & 0.001 & $\mathrm{~L}$ \\
\hline 28.6083 & 0.002 & 0.002 & $\mathrm{~L}$ & 31.94116 & 0.001 & 0.001 & $\mathrm{H}$ \\
\hline 28.622 & 0.002 & 0.002 & $\mathrm{~L}$ & 31.973 & 0.001 & 0.001 & $\mathrm{H}$ \\
\hline 28.64991 & 0.001 & 0.001 & $\mathrm{~L}$ & 31.9995 & 0.001 & 0.001 & $\mathrm{~L}$ \\
\hline 28.9995 & 0.001 & 0.001 & $\mathrm{~L}$ & 32.0 & 0.0005 & 0.0005 & $\mathrm{H}$ \\
\hline 29.0 & 0.001 & 0.001 & $\mathrm{H}$ & 32.0 & 0.001 & 0.001 & $\mathrm{H}$ \\
\hline 29.03770625 & 0.001 & 0.001 & $\mathrm{H}$ & 32.24978 & 0.001 & 0.001 & $\mathrm{~L}$ \\
\hline 29.0392375 & 0.001 & 0.001 & $\mathrm{H}$ & 32.24987 & 0.001 & 0.001 & $\mathrm{H}$ \\
\hline 29.1431 & 0.001 & 0.001 & $\mathrm{~L}$ & 32.499844 & 0.001 & 0.001 & $\mathrm{~L}$ \\
\hline 29.1698 & 0.001 & 0.001 & $\mathrm{~L}$ & 32.5 & 0.001 & 0.001 & $\mathrm{H}$ \\
\hline 29.19603 & 0.001 & 0.001 & $\mathrm{~L}$ & 33.7 & 0.01556 & 0.01556 & $\mathrm{~L}$ \\
\hline 29.19603 & 0.001 & 0.001 & $\mathrm{~L}$ & 33.8 & 0.0005 & 0.0005 & $\mathrm{~L}$ \\
\hline 29.223 & 0.001 & 0.001 & $\mathrm{~L}$ & 34.3 & 0.0005 & 0.0005 & $\mathrm{~L}$ \\
\hline 29.2495 & 0.001 & 0.001 & $\mathrm{~L}$ & 34.7 & 0.02778 & 0.02778 & $\mathrm{H}$ \\
\hline 29.24987 & 0.001 & 0.001 & $\mathrm{~L}$ & 34.7 & 0.13 & 0.13 & $\mathrm{~L}$ \\
\hline 29.2499 & 0.001 & 0.001 & $\mathrm{H}$ & 35.3 & 0.02778 & 0.02778 & $\mathrm{H}$ \\
\hline 29.2767 & 0.001 & 0.001 & $\mathrm{~L}$ & 35.3 & 0.13 & 0.13 & $\mathrm{~L}$ \\
\hline 29.3031 & 0.001 & 0.001 & $\mathrm{~L}$ & 35.706385 & 0.003055 & 0.003055 & $\mathrm{~L}$ \\
\hline 29.499883 & 0.001 & 0.001 & $\mathrm{~L}$ & 35.7095265 & 0.01222 & 0.01222 & $\mathrm{H}$ \\
\hline 29.5 & 0.001 & 0.001 & $\mathrm{H}$ & 35.9 & 0.10222 & 0.10222 & $\mathrm{H}$ \\
\hline 29.5948 & 0.002 & 0.002 & $\mathrm{~L}$ & 35.958055 & 0.009165 & 0.009165 & $\mathrm{~L}$ \\
\hline 29.6085 & 0.002 & 0.002 & $\mathrm{~L}$ & 36.7 & 0.10722 & 0.10722 & $\mathrm{H}$ \\
\hline 29.63784 & 0.001 & 0.001 & $\mathrm{~L}$ & 36.7 & 0.0005 & 0.0005 & $\mathrm{~L}$ \\
\hline 29.9995 & 0.001 & 0.001 & $\mathrm{~L}$ & 37.3 & 0.01 & 0.01 & $\mathrm{H}$ \\
\hline 30.0 & 0.001 & 0.001 & $\mathrm{H}$ & 38.955 & 0.001 & 0.001 & $\mathrm{~L}$ \\
\hline 30.24984 & 0.001 & 0.001 & $\mathrm{~L}$ & 38.9674 & 0.001 & 0.001 & $\mathrm{H}$ \\
\hline 30.24989 & 0.001 & 0.001 & $\mathrm{H}$ & 38.9815 & 0.001 & 0.001 & $\mathrm{~L}$ \\
\hline 30.49987 & 0.001 & 0.001 & $\mathrm{~L}$ & 38.9995 & 0.001 & 0.001 & $\mathrm{~L}$ \\
\hline 30.5 & 0.001 & 0.001 & $\mathrm{H}$ & 39.0 & 0.001 & 0.001 & $\mathrm{H}$ \\
\hline 30.5813 & 0.002 & 0.002 & $\mathrm{~L}$ & 39.0087 & 0.001 & 0.001 & $\mathrm{~L}$ \\
\hline 30.595 & 0.002 & 0.002 & $\mathrm{~L}$ & 39.0351 & 0.001 & 0.001 & $\mathrm{~L}$ \\
\hline 30.62577 & 0.001 & 0.001 & $\mathrm{~L}$ & 39.24957 & 0.001 & 0.001 & $\mathrm{~L}$ \\
\hline 30.943 & 0.001 & 0.001 & $\mathrm{H}$ & 39.2498 & 0.001 & 0.001 & $\mathrm{H}$ \\
\hline 30.9738 & 0.001 & 0.001 & $\mathrm{H}$ & 39.408315625 & 0.001 & 0.001 & $\mathrm{H}$ \\
\hline 30.9995 & 0.001 & 0.001 & $\mathrm{~L}$ & 39.41039375 & 0.001 & 0.001 & $\mathrm{H}$ \\
\hline 31.0 & 0.001 & 0.001 & $\mathrm{H}$ & 39.4598 & 0.002 & 0.002 & $\mathrm{~L}$ \\
\hline 31.0895 & 0.001 & 0.001 & $\mathrm{~L}$ & 39.4735 & 0.002 & 0.002 & $\mathrm{~L}$ \\
\hline 31.111828125 & 0.001 & 0.001 & $\mathrm{H}$ & 39.499753 & 0.001 & 0.001 & $\mathrm{~L}$ \\
\hline 31.11346875 & 0.001 & 0.001 & $\mathrm{H}$ & 39.5 & 0.001 & 0.001 & $\mathrm{H}$ \\
\hline 31.1162 & 0.001 & 0.001 & $\mathrm{~L}$ & 39.51714 & 0.001 & 0.001 & $\mathrm{~L}$ \\
\hline 31.14243 & 0.001 & 0.001 & $\mathrm{~L}$ & 39.6 & 0.0005 & 0.0005 & $\mathrm{~L}$ \\
\hline
\end{tabular}

TABLE VII. (Continued) 
TABLE VII. (Continued)

\begin{tabular}{|c|c|c|c|c|c|c|c|}
\hline$\underline{f_{\mathrm{L}}(\mathrm{Hz})}$ & LFS (Hz) & HFS (Hz) & IFO & $\underline{f_{\mathrm{L}}(\mathrm{Hz})}$ & LFS $(\mathrm{Hz})$ & HFS (Hz) & IFO \\
\hline 39.92644 & 0.001 & 0.001 & $\mathrm{H}$ & 42.9995 & 0.001 & 0.001 & $\mathrm{~L}$ \\
\hline 39.9666 & 0.001 & 0.001 & $\mathrm{H}$ & 43.0 & 0.001 & 0.001 & $\mathrm{H}$ \\
\hline 39.9995 & 0.001 & 0.001 & $\mathrm{~L}$ & 43.24945 & 0.001 & 0.001 & $\mathrm{~L}$ \\
\hline 40.0 & 0.0005 & 0.0005 & $\mathrm{H}$ & 43.24976 & 0.001 & 0.001 & $\mathrm{H}$ \\
\hline 40.0 & 0.001 & 0.001 & $\mathrm{H}$ & 43.4058 & 0.002 & 0.002 & $\mathrm{~L}$ \\
\hline 40.24954 & 0.001 & 0.001 & $\mathrm{~L}$ & 43.4195 & 0.002 & 0.002 & $\mathrm{~L}$ \\
\hline 40.24979 & 0.001 & 0.001 & $\mathrm{H}$ & 43.46886 & 0.001 & 0.001 & $\mathrm{~L}$ \\
\hline 40.4463 & 0.002 & 0.002 & $\mathrm{~L}$ & 43.499701 & 0.001 & 0.001 & $\mathrm{~L}$ \\
\hline 40.46 & 0.002 & 0.002 & $\mathrm{~L}$ & 43.5 & 0.001 & 0.001 & $\mathrm{H}$ \\
\hline 40.49974 & 0.001 & 0.001 & $\mathrm{~L}$ & 43.556559375 & 0.001 & 0.001 & $\mathrm{H}$ \\
\hline 40.5 & 0.001 & 0.001 & $\mathrm{H}$ & 43.55885625 & 0.001 & 0.001 & $\mathrm{H}$ \\
\hline 40.50507 & 0.001 & 0.001 & $\mathrm{~L}$ & 43.91908 & 0.001 & 0.001 & $\mathrm{H}$ \\
\hline 40.8215 & 0.001 & 0.001 & $\mathrm{~L}$ & 43.9634 & 0.001 & 0.001 & $\mathrm{H}$ \\
\hline 40.8482 & 0.001 & 0.001 & $\mathrm{~L}$ & 43.9995 & 0.001 & 0.001 & $\mathrm{~L}$ \\
\hline 40.87443 & 0.001 & 0.001 & $\mathrm{~L}$ & 44.0 & 0.001 & 0.001 & $\mathrm{H}$ \\
\hline 40.87443 & 0.001 & 0.001 & $\mathrm{~L}$ & 44.24942 & 0.001 & 0.001 & $\mathrm{~L}$ \\
\hline 40.9014 & 0.001 & 0.001 & $\mathrm{~L}$ & 44.24975 & 0.001 & 0.001 & $\mathrm{H}$ \\
\hline 40.9246 & 0.001 & 0.001 & $\mathrm{H}$ & 44.3923 & 0.002 & 0.002 & $\mathrm{~L}$ \\
\hline 40.9279 & 0.001 & 0.001 & $\mathrm{~L}$ & 44.406 & 0.002 & 0.002 & $\mathrm{~L}$ \\
\hline 40.9551 & 0.001 & 0.001 & $\mathrm{~L}$ & 44.45679 & 0.001 & 0.001 & $\mathrm{~L}$ \\
\hline 40.9658 & 0.001 & 0.001 & $\mathrm{H}$ & 44.499688 & 0.001 & 0.001 & $\mathrm{~L}$ \\
\hline 40.9815 & 0.001 & 0.001 & $\mathrm{~L}$ & 44.5 & 0.001 & 0.001 & $\mathrm{H}$ \\
\hline 40.9995 & 0.001 & 0.001 & $\mathrm{~L}$ & 44.7143 & 0.001 & 0.001 & $\mathrm{~L}$ \\
\hline 41.0 & 0.001 & 0.001 & $\mathrm{H}$ & 44.741 & 0.001 & 0.001 & $\mathrm{~L}$ \\
\hline 41.24951 & 0.001 & 0.001 & $\mathrm{~L}$ & 44.76723 & 0.001 & 0.001 & $\mathrm{~L}$ \\
\hline 41.24978 & 0.001 & 0.001 & $\mathrm{H}$ & 44.76723 & 0.001 & 0.001 & $\mathrm{~L}$ \\
\hline 41.4328 & 0.002 & 0.002 & $\mathrm{~L}$ & 44.7942 & 0.001 & 0.001 & $\mathrm{~L}$ \\
\hline 41.4465 & 0.002 & 0.002 & $\mathrm{~L}$ & 44.8207 & 0.001 & 0.001 & $\mathrm{~L}$ \\
\hline 41.4824375 & 0.001 & 0.001 & $\mathrm{H}$ & 44.8479 & 0.001 & 0.001 & $\mathrm{~L}$ \\
\hline 41.484625 & 0.001 & 0.001 & $\mathrm{H}$ & 44.8743 & 0.001 & 0.001 & $\mathrm{~L}$ \\
\hline 41.493 & 0.001 & 0.001 & $\mathrm{~L}$ & 44.91724 & 0.001 & 0.001 & $\mathrm{H}$ \\
\hline 41.499727 & 0.001 & 0.001 & $\mathrm{~L}$ & 44.9626 & 0.001 & 0.001 & $\mathrm{H}$ \\
\hline 41.5 & 0.001 & 0.001 & $\mathrm{H}$ & 44.9995 & 0.001 & 0.001 & $\mathrm{~L}$ \\
\hline 41.92276 & 0.001 & 0.001 & $\mathrm{H}$ & 45.0 & 0.001 & 0.001 & $\mathrm{H}$ \\
\hline 41.965 & 0.001 & 0.001 & $\mathrm{H}$ & 45.24939 & 0.001 & 0.001 & $\mathrm{~L}$ \\
\hline 41.9995 & 0.001 & 0.001 & $\mathrm{~L}$ & 45.24974 & 0.001 & 0.001 & $\mathrm{H}$ \\
\hline 42.0 & 0.001 & 0.001 & $\mathrm{H}$ & 45.3788 & 0.002 & 0.002 & $\mathrm{~L}$ \\
\hline 42.24948 & 0.001 & 0.001 & $\mathrm{~L}$ & 45.3925 & 0.002 & 0.002 & $\mathrm{~L}$ \\
\hline 42.24977 & 0.001 & 0.001 & $\mathrm{H}$ & 45.4 & 0.0005 & 0.0005 & $\mathrm{~L}$ \\
\hline 42.4193 & 0.002 & 0.002 & $\mathrm{~L}$ & 45.44472 & 0.001 & 0.001 & $\mathrm{~L}$ \\
\hline 42.433 & 0.002 & 0.002 & $\mathrm{~L}$ & 45.499675 & 0.001 & 0.001 & $\mathrm{~L}$ \\
\hline 42.48093 & 0.001 & 0.001 & $\mathrm{~L}$ & 45.5 & 0.001 & 0.001 & $\mathrm{H}$ \\
\hline 42.499714 & 0.001 & 0.001 & $\mathrm{~L}$ & 45.63068125 & 0.001 & 0.001 & $\mathrm{H}$ \\
\hline 42.5 & 0.001 & 0.001 & $\mathrm{H}$ & 45.6330875 & 0.001 & 0.001 & $\mathrm{H}$ \\
\hline 42.5 & 0.0005 & 0.0005 & $\mathrm{~L}$ & 45.9 & 0.0005 & 0.0005 & $\mathrm{~L}$ \\
\hline 42.7679 & 0.001 & 0.001 & $\mathrm{~L}$ & 45.9154 & 0.001 & 0.001 & $\mathrm{H}$ \\
\hline 42.7946 & 0.001 & 0.001 & $\mathrm{~L}$ & 45.9618 & 0.001 & 0.001 & $\mathrm{H}$ \\
\hline 42.82083 & 0.001 & 0.001 & $\mathrm{~L}$ & 45.9995 & 0.001 & 0.001 & $\mathrm{~L}$ \\
\hline 42.82083 & 0.001 & 0.001 & $\mathrm{~L}$ & 46.0 & 0.001 & 0.001 & $\mathrm{H}$ \\
\hline 42.8478 & 0.001 & 0.001 & $\mathrm{~L}$ & 46.24936 & 0.001 & 0.001 & $\mathrm{~L}$ \\
\hline 42.8743 & 0.001 & 0.001 & $\mathrm{~L}$ & 46.24973 & 0.001 & 0.001 & $\mathrm{H}$ \\
\hline 42.9015 & 0.001 & 0.001 & $\mathrm{~L}$ & 46.3653 & 0.002 & 0.002 & $\mathrm{~L}$ \\
\hline 42.92092 & 0.001 & 0.001 & $\mathrm{H}$ & 46.379 & 0.002 & 0.002 & $\mathrm{~L}$ \\
\hline 42.9279 & 0.001 & 0.001 & $\mathrm{~L}$ & 46.43265 & 0.001 & 0.001 & $\mathrm{~L}$ \\
\hline 42.9642 & 0.001 & 0.001 & $\mathrm{H}$ & 46.499662 & 0.001 & 0.001 & $\mathrm{~L}$ \\
\hline
\end{tabular}

TABLE VII. (Continued)

(Table continued) 
TABLE VII. (Continued)

\begin{tabular}{|c|c|c|c|c|c|c|c|}
\hline$\underline{f_{\mathrm{L}}(\mathrm{Hz})}$ & LFS $(\mathrm{Hz})$ & $\mathrm{HFS}(\mathrm{Hz})$ & IFO & $\underline{f_{\mathrm{L}}(\mathrm{Hz})}$ & LFS $(\mathrm{Hz})$ & $\mathrm{HFS}(\mathrm{Hz})$ & IFO \\
\hline 46.5 & 0.001 & 0.001 & $\mathrm{H}$ & 49.778925 & 0.001 & 0.001 & $\mathrm{H}$ \\
\hline 46.6607 & 0.001 & 0.001 & $\mathrm{~L}$ & 49.78155 & 0.001 & 0.001 & $\mathrm{H}$ \\
\hline 46.6874 & 0.001 & 0.001 & $\mathrm{~L}$ & 49.90804 & 0.001 & 0.001 & $\mathrm{H}$ \\
\hline 46.71363 & 0.001 & 0.001 & $\mathrm{~L}$ & 49.9995 & 0.001 & 0.001 & $\mathrm{~L}$ \\
\hline 46.71363 & 0.001 & 0.001 & $\mathrm{~L}$ & 50.0 & 0.001 & 0.001 & $\mathrm{H}$ \\
\hline 46.7406 & 0.001 & 0.001 & $\mathrm{~L}$ & 50.24924 & 0.001 & 0.001 & $\mathrm{~L}$ \\
\hline 46.7671 & 0.001 & 0.001 & $\mathrm{~L}$ & 50.3113 & 0.002 & 0.002 & $\mathrm{~L}$ \\
\hline 46.7943 & 0.001 & 0.001 & $\mathrm{~L}$ & 50.325 & 0.002 & 0.002 & $\mathrm{~L}$ \\
\hline 46.8207 & 0.001 & 0.001 & $\mathrm{~L}$ & 50.49961 & 0.001 & 0.001 & $\mathrm{~L}$ \\
\hline 46.91356 & 0.001 & 0.001 & $\mathrm{H}$ & 50.5 & 0.001 & 0.001 & $\mathrm{H}$ \\
\hline 46.961 & 0.001 & 0.001 & $\mathrm{H}$ & 50.5535 & 0.001 & 0.001 & $\mathrm{~L}$ \\
\hline 46.9995 & 0.001 & 0.001 & $\mathrm{~L}$ & 50.5802 & 0.001 & 0.001 & $\mathrm{~L}$ \\
\hline 47.0 & 0.001 & 0.001 & $\mathrm{H}$ & 50.60643 & 0.001 & 0.001 & $\mathrm{~L}$ \\
\hline 47.24933 & 0.001 & 0.001 & $\mathrm{~L}$ & 50.60643 & 0.001 & 0.001 & $\mathrm{~L}$ \\
\hline 47.24972 & 0.001 & 0.001 & $\mathrm{H}$ & 50.6334 & 0.001 & 0.001 & $\mathrm{~L}$ \\
\hline 47.3518 & 0.002 & 0.002 & $\mathrm{~L}$ & 50.6599 & 0.001 & 0.001 & $\mathrm{~L}$ \\
\hline 47.3655 & 0.002 & 0.002 & $\mathrm{~L}$ & 50.6871 & 0.001 & 0.001 & $\mathrm{~L}$ \\
\hline 47.42058 & 0.001 & 0.001 & $\mathrm{~L}$ & 50.7135 & 0.001 & 0.001 & $\mathrm{~L}$ \\
\hline 47.499649 & 0.001 & 0.001 & $\mathrm{~L}$ & 50.9062 & 0.001 & 0.001 & $\mathrm{H}$ \\
\hline 47.5 & 0.001 & 0.001 & $\mathrm{H}$ & 51.0 & 0.001 & 0.001 & $\mathrm{H}$ \\
\hline 47.704803125 & 0.001 & 0.001 & $\mathrm{H}$ & 51.2 & 0.0005 & 0.0005 & $\mathrm{~L}$ \\
\hline 47.70731875 & 0.001 & 0.001 & $\mathrm{H}$ & 51.24921 & 0.001 & 0.001 & $\mathrm{~L}$ \\
\hline 47.8 & 0.0005 & 0.0005 & $\mathrm{~L}$ & 51.2978 & 0.002 & 0.002 & $\mathrm{~L}$ \\
\hline 47.91172 & 0.001 & 0.001 & $\mathrm{H}$ & 51.3115 & 0.002 & 0.002 & $\mathrm{~L}$ \\
\hline 47.94158 & 0.0032 & 0.0016 & $\mathrm{~L}$ & 51.499597 & 0.001 & 0.001 & $\mathrm{~L}$ \\
\hline 47.9602 & 0.001 & 0.001 & $\mathrm{H}$ & 51.5 & 0.001 & 0.001 & $\mathrm{H}$ \\
\hline 47.9995 & 0.001 & 0.001 & $\mathrm{~L}$ & 51.853046875 & 0.001 & 0.001 & $\mathrm{H}$ \\
\hline 48.0 & 0.0005 & 0.0005 & $\mathrm{H}$ & 51.85578125 & 0.001 & 0.001 & $\mathrm{H}$ \\
\hline 48.0 & 0.001 & 0.001 & $\mathrm{H}$ & 51.90436 & 0.001 & 0.001 & $\mathrm{H}$ \\
\hline 48.2493 & 0.001 & 0.001 & $\mathrm{~L}$ & 52.0 & 0.001 & 0.001 & $\mathrm{H}$ \\
\hline 48.24971 & 0.001 & 0.001 & $\mathrm{H}$ & 52.24918 & 0.001 & 0.001 & $\mathrm{~L}$ \\
\hline 48.3 & 0.0005 & 0.0005 & $\mathrm{~L}$ & 52.2843 & 0.002 & 0.002 & $\mathrm{~L}$ \\
\hline 48.3383 & 0.002 & 0.002 & $\mathrm{~L}$ & 52.298 & 0.002 & 0.002 & $\mathrm{~L}$ \\
\hline 48.352 & 0.002 & 0.002 & $\mathrm{~L}$ & 52.499584 & 0.001 & 0.001 & $\mathrm{~L}$ \\
\hline 48.40851 & 0.001 & 0.001 & $\mathrm{~L}$ & 52.4999 & 0.001 & 0.001 & $\mathrm{~L}$ \\
\hline 48.499636 & 0.001 & 0.001 & $\mathrm{~L}$ & 52.5 & 0.001 & 0.001 & $\mathrm{H}$ \\
\hline 48.5 & 0.001 & 0.001 & $\mathrm{H}$ & 52.5266 & 0.001 & 0.001 & $\mathrm{~L}$ \\
\hline 48.6071 & 0.001 & 0.001 & $\mathrm{~L}$ & 52.55283 & 0.001 & 0.001 & $\mathrm{~L}$ \\
\hline 48.6338 & 0.001 & 0.001 & $\mathrm{~L}$ & 52.55283 & 0.001 & 0.001 & $\mathrm{~L}$ \\
\hline 48.66003 & 0.001 & 0.001 & $\mathrm{~L}$ & 52.5798 & 0.001 & 0.001 & $\mathrm{~L}$ \\
\hline 48.66003 & 0.001 & 0.001 & $\mathrm{~L}$ & 52.6063 & 0.001 & 0.001 & $\mathrm{~L}$ \\
\hline 48.687 & 0.001 & 0.001 & $\mathrm{~L}$ & 52.6335 & 0.001 & 0.001 & $\mathrm{~L}$ \\
\hline 48.7135 & 0.001 & 0.001 & $\mathrm{~L}$ & 52.6599 & 0.001 & 0.001 & $\mathrm{~L}$ \\
\hline 48.7407 & 0.001 & 0.001 & $\mathrm{~L}$ & 52.90252 & 0.001 & 0.001 & $\mathrm{H}$ \\
\hline 48.7671 & 0.001 & 0.001 & $\mathrm{~L}$ & 53.0 & 0.001 & 0.001 & $\mathrm{H}$ \\
\hline 48.90988 & 0.001 & 0.001 & $\mathrm{H}$ & 53.24915 & 0.001 & 0.001 & $\mathrm{~L}$ \\
\hline 48.9594 & 0.001 & 0.001 & $\mathrm{H}$ & 53.2708 & 0.002 & 0.002 & $\mathrm{~L}$ \\
\hline 48.9995 & 0.001 & 0.001 & $\mathrm{~L}$ & 53.2845 & 0.002 & 0.002 & $\mathrm{~L}$ \\
\hline 49.0 & 0.001 & 0.001 & $\mathrm{H}$ & 53.499571 & 0.001 & 0.001 & $\mathrm{~L}$ \\
\hline 49.24927 & 0.001 & 0.001 & $\mathrm{~L}$ & 53.5 & 0.001 & 0.001 & $\mathrm{H}$ \\
\hline 49.2497 & 0.001 & 0.001 & $\mathrm{H}$ & 53.90068 & 0.001 & 0.001 & $\mathrm{H}$ \\
\hline 49.3248 & 0.002 & 0.002 & $\mathrm{~L}$ & 53.92716875 & 0.001 & 0.001 & $\mathrm{H}$ \\
\hline 49.3385 & 0.002 & 0.002 & $\mathrm{~L}$ & 53.9300125 & 0.001 & 0.001 & $\mathrm{H}$ \\
\hline 49.499623 & 0.001 & 0.001 & $\mathrm{~L}$ & 54.0 & 0.001 & 0.001 & $\mathrm{H}$ \\
\hline 49.5 & 0.001 & 0.001 & $\mathrm{H}$ & 54.1 & 0.0005 & 0.0005 & $\mathrm{~L}$ \\
\hline
\end{tabular}

TABLE VII. (Continued) 
TABLE VII. (Continued)

\begin{tabular}{|c|c|c|c|c|c|c|c|}
\hline$f_{\mathrm{L}}(\mathrm{Hz})$ & LFS $(\mathrm{Hz})$ & HFS (Hz) & IFO & $\underline{f_{\mathrm{L}}(\mathrm{Hz})}$ & LFS $(\mathrm{Hz})$ & HFS (Hz) & IFO \\
\hline 54.2573 & 0.002 & 0.002 & $\mathrm{~L}$ & 59.88964 & 0.001 & 0.001 & $\mathrm{H}$ \\
\hline 54.271 & 0.002 & 0.002 & $\mathrm{~L}$ & 59.926975 & 0.004 & 0.002 & $\mathrm{~L}$ \\
\hline 54.4463 & 0.001 & 0.001 & $\mathrm{~L}$ & 60.0 & 0.001 & 0.001 & $\mathrm{H}$ \\
\hline 54.473 & 0.001 & 0.001 & $\mathrm{~L}$ & 60.0 & 0.06 & 0.06 & $\mathrm{H}$ \\
\hline 54.49923 & 0.001 & 0.001 & $\mathrm{~L}$ & 60.0 & 0.06 & 0.06 & $\mathrm{~L}$ \\
\hline 54.49923 & 0.001 & 0.001 & $\mathrm{~L}$ & 60.149534375 & 0.001 & 0.001 & $\mathrm{H}$ \\
\hline 54.499558 & 0.001 & 0.001 & $\mathrm{~L}$ & 60.15270625 & 0.001 & 0.001 & $\mathrm{H}$ \\
\hline 54.5 & 0.001 & 0.001 & $\mathrm{H}$ & 60.2855 & 0.001 & 0.001 & $\mathrm{~L}$ \\
\hline 54.5262 & 0.001 & 0.001 & $\mathrm{~L}$ & 60.3122 & 0.001 & 0.001 & $\mathrm{~L}$ \\
\hline 54.5527 & 0.001 & 0.001 & $\mathrm{~L}$ & 60.33843 & 0.001 & 0.001 & $\mathrm{~L}$ \\
\hline 54.5799 & 0.001 & 0.001 & $\mathrm{~L}$ & 60.33843 & 0.001 & 0.001 & $\mathrm{~L}$ \\
\hline 54.6063 & 0.001 & 0.001 & $\mathrm{~L}$ & 60.3654 & 0.001 & 0.001 & $\mathrm{~L}$ \\
\hline 54.89884 & 0.001 & 0.001 & $\mathrm{H}$ & 60.3919 & 0.001 & 0.001 & $\mathrm{~L}$ \\
\hline 55.0 & 0.001 & 0.001 & $\mathrm{H}$ & 60.49948 & 0.001 & 0.001 & $\mathrm{~L}$ \\
\hline 55.2438 & 0.002 & 0.002 & $\mathrm{~L}$ & 60.5 & 0.001 & 0.001 & $\mathrm{H}$ \\
\hline 55.2575 & 0.002 & 0.002 & $\mathrm{~L}$ & 60.8878 & 0.001 & 0.001 & $\mathrm{H}$ \\
\hline 55.499545 & 0.001 & 0.001 & $\mathrm{~L}$ & 61.0 & 0.001 & 0.001 & $\mathrm{H}$ \\
\hline 55.5 & 0.001 & 0.001 & $\mathrm{H}$ & 61.499467 & 0.001 & 0.001 & $\mathrm{~L}$ \\
\hline 55.897 & 0.001 & 0.001 & $\mathrm{H}$ & 61.5 & 0.001 & 0.001 & $\mathrm{H}$ \\
\hline 56.0 & 0.0005 & 0.0005 & $\mathrm{H}$ & 62.0 & 0.001 & 0.001 & $\mathrm{H}$ \\
\hline 56.0 & 0.001 & 0.001 & $\mathrm{H}$ & 62.22365625 & 0.001 & 0.001 & $\mathrm{H}$ \\
\hline 56.001290625 & 0.001 & 0.001 & $\mathrm{H}$ & 62.2269375 & 0.001 & 0.001 & $\mathrm{H}$ \\
\hline 56.00424375 & 0.001 & 0.001 & $\mathrm{H}$ & 62.28483 & 0.001 & 0.001 & $\mathrm{~L}$ \\
\hline 56.3927 & 0.001 & 0.001 & $\mathrm{~L}$ & 62.28483 & 0.001 & 0.001 & $\mathrm{~L}$ \\
\hline 56.4194 & 0.001 & 0.001 & $\mathrm{~L}$ & 62.3 & 0.0005 & 0.0005 & $\mathrm{~L}$ \\
\hline 56.44563 & 0.001 & 0.001 & $\mathrm{~L}$ & 62.499454 & 0.001 & 0.001 & $\mathrm{~L}$ \\
\hline 56.44563 & 0.001 & 0.001 & $\mathrm{~L}$ & 62.5 & 0.001 & 0.001 & $\mathrm{H}$ \\
\hline 56.4726 & 0.001 & 0.001 & $\mathrm{~L}$ & 62.8 & 0.0005 & 0.0005 & $\mathrm{~L}$ \\
\hline 56.4991 & 0.001 & 0.001 & $\mathrm{~L}$ & 62.8254 & 0.003 & 0.003 & $\mathrm{H}$ \\
\hline 56.499532 & 0.001 & 0.001 & $\mathrm{~L}$ & 62.8298 & 0.003 & 0.003 & $\mathrm{H}$ \\
\hline 56.5 & 0.001 & 0.001 & $\mathrm{H}$ & 63.0 & 0.001 & 0.001 & $\mathrm{H}$ \\
\hline 56.5 & 0.0005 & 0.0005 & $\mathrm{~L}$ & 63.499441 & 0.001 & 0.001 & $\mathrm{~L}$ \\
\hline 56.5263 & 0.001 & 0.001 & $\mathrm{~L}$ & 63.5 & 0.001 & 0.001 & $\mathrm{H}$ \\
\hline 56.5527 & 0.001 & 0.001 & $\mathrm{~L}$ & 64.0 & 0.0005 & 0.0005 & $\mathrm{H}$ \\
\hline 56.89516 & 0.001 & 0.001 & $\mathrm{H}$ & 64.0 & 0.001 & 0.001 & $\mathrm{H}$ \\
\hline 57.0 & 0.001 & 0.001 & $\mathrm{H}$ & 64.297778125 & 0.001 & 0.001 & $\mathrm{H}$ \\
\hline 57.0 & 0.0005 & 0.0005 & $\mathrm{~L}$ & 64.30116875 & 0.001 & 0.001 & $\mathrm{H}$ \\
\hline 57.499519 & 0.001 & 0.001 & $\mathrm{~L}$ & 64.499428 & 0.001 & 0.001 & $\mathrm{~L}$ \\
\hline 57.5 & 0.001 & 0.001 & $\mathrm{H}$ & 64.5 & 0.001 & 0.001 & $\mathrm{H}$ \\
\hline 57.89332 & 0.001 & 0.001 & $\mathrm{H}$ & 65.0 & 0.001 & 0.001 & $\mathrm{H}$ \\
\hline 58.0 & 0.001 & 0.001 & $\mathrm{H}$ & 65.2 & 0.0005 & 0.0005 & $\mathrm{~L}$ \\
\hline 58.0754125 & 0.001 & 0.001 & $\mathrm{H}$ & 65.499415 & 0.001 & 0.001 & $\mathrm{~L}$ \\
\hline 58.078475 & 0.001 & 0.001 & $\mathrm{H}$ & 65.5 & 0.001 & 0.001 & $\mathrm{H}$ \\
\hline 58.3391 & 0.001 & 0.001 & $\mathrm{~L}$ & 65.7 & 0.0005 & 0.0005 & $\mathrm{~L}$ \\
\hline 58.3658 & 0.001 & 0.001 & $\mathrm{~L}$ & 66.0 & 0.001 & 0.001 & $\mathrm{H}$ \\
\hline 58.39203 & 0.001 & 0.001 & $\mathrm{~L}$ & 66.3719 & 0.001 & 0.001 & $\mathrm{H}$ \\
\hline 58.39203 & 0.001 & 0.001 & $\mathrm{~L}$ & 66.3754 & 0.001 & 0.001 & $\mathrm{H}$ \\
\hline 58.419 & 0.001 & 0.001 & $\mathrm{~L}$ & 66.499402 & 0.001 & 0.001 & $\mathrm{~L}$ \\
\hline 58.4455 & 0.001 & 0.001 & $\mathrm{~L}$ & 66.5 & 0.001 & 0.001 & $\mathrm{H}$ \\
\hline 58.499506 & 0.001 & 0.001 & $\mathrm{~L}$ & 66.665 & 0.001 & 0.001 & $\mathrm{~L}$ \\
\hline 58.5 & 0.001 & 0.001 & $\mathrm{H}$ & 67.0 & 0.001 & 0.001 & $\mathrm{H}$ \\
\hline 58.89148 & 0.001 & 0.001 & $\mathrm{H}$ & 67.499389 & 0.001 & 0.001 & $\mathrm{~L}$ \\
\hline 59.0 & 0.001 & 0.001 & $\mathrm{H}$ & 67.5 & 0.001 & 0.001 & $\mathrm{H}$ \\
\hline 59.499493 & 0.001 & 0.001 & $\mathrm{~L}$ & 67.6 & 0.0005 & 0.0005 & $\mathrm{~L}$ \\
\hline 59.5 & 0.001 & 0.001 & $\mathrm{H}$ & 68.0 & 0.001 & 0.001 & $\mathrm{H}$ \\
\hline
\end{tabular}

TABLE VII. (Continued) 
TABLE VII. (Continued)

\begin{tabular}{|c|c|c|c|c|c|c|c|}
\hline$f_{\mathrm{L}}(\mathrm{Hz})$ & LFS $(\mathrm{Hz})$ & $\mathrm{HFS}(\mathrm{Hz})$ & IFO & $f_{\mathrm{L}}(\mathrm{Hz})$ & LFS $(\mathrm{Hz})$ & $\mathrm{HFS}(\mathrm{Hz})$ & IFO \\
\hline 68.1 & 0.0005 & 0.0005 & $\mathrm{~L}$ & 78.5 & 0.001 & 0.001 & $\mathrm{H}$ \\
\hline 68.499376 & 0.001 & 0.001 & $\mathrm{~L}$ & 78.74995 & 0.001 & 0.001 & $\mathrm{~L}$ \\
\hline 68.5 & 0.001 & 0.001 & $\mathrm{H}$ & 79.0 & 0.001 & 0.001 & $\mathrm{H}$ \\
\hline 68.6 & 0.0005 & 0.0005 & $\mathrm{~L}$ & 79.2 & 0.0005 & 0.0005 & $\mathrm{~L}$ \\
\hline 69.0 & 0.001 & 0.001 & $\mathrm{H}$ & 79.5 & 0.001 & 0.001 & $\mathrm{H}$ \\
\hline 69.499363 & 0.001 & 0.001 & $\mathrm{~L}$ & 79.7 & 0.0005 & 0.0005 & $\mathrm{~L}$ \\
\hline 69.5 & 0.001 & 0.001 & $\mathrm{H}$ & 79.749925 & 0.001 & 0.001 & $\mathrm{~L}$ \\
\hline 70.0 & 0.001 & 0.001 & $\mathrm{H}$ & 80.0 & 0.0005 & 0.0005 & $\mathrm{H}$ \\
\hline 70.49935 & 0.001 & 0.001 & $\mathrm{~L}$ & 80.0 & 0.001 & 0.001 & $\mathrm{H}$ \\
\hline 70.5 & 0.001 & 0.001 & $\mathrm{H}$ & 80.5 & 0.001 & 0.001 & $\mathrm{H}$ \\
\hline 71.0 & 0.001 & 0.001 & $\mathrm{H}$ & 80.7499 & 0.001 & 0.001 & $\mathrm{~L}$ \\
\hline 71.0 & 0.0005 & 0.0005 & $\mathrm{~L}$ & 81.0 & 0.001 & 0.001 & $\mathrm{H}$ \\
\hline 71.499337 & 0.001 & 0.001 & $\mathrm{~L}$ & 81.5 & 0.001 & 0.001 & $\mathrm{H}$ \\
\hline 71.5 & 0.001 & 0.001 & $\mathrm{H}$ & 81.749875 & 0.001 & 0.001 & $\mathrm{~L}$ \\
\hline 71.5 & 0.0005 & 0.0005 & $\mathrm{~L}$ & 82.0 & 0.001 & 0.001 & $\mathrm{H}$ \\
\hline 71.91237 & 0.0048 & 0.0024 & $\mathrm{~L}$ & 82.1 & 0.0005 & 0.0005 & $\mathrm{~L}$ \\
\hline 72.0 & 0.0005 & 0.0005 & $\mathrm{H}$ & 82.5 & 0.001 & 0.001 & $\mathrm{H}$ \\
\hline 72.0 & 0.001 & 0.001 & $\mathrm{H}$ & 82.6 & 0.0005 & 0.0005 & $\mathrm{~L}$ \\
\hline 72.499324 & 0.001 & 0.001 & $\mathrm{~L}$ & 82.74985 & 0.001 & 0.001 & $\mathrm{~L}$ \\
\hline 72.5 & 0.001 & 0.001 & $\mathrm{H}$ & 83.0 & 0.001 & 0.001 & $\mathrm{H}$ \\
\hline 73.0 & 0.001 & 0.001 & $\mathrm{H}$ & 83.5 & 0.001 & 0.001 & $\mathrm{H}$ \\
\hline 73.499311 & 0.001 & 0.001 & $\mathrm{~L}$ & 83.749825 & 0.001 & 0.001 & $\mathrm{~L}$ \\
\hline 73.5 & 0.001 & 0.001 & $\mathrm{H}$ & 83.897765 & 0.0056 & 0.0028 & $\mathrm{~L}$ \\
\hline 73.9 & 0.0005 & 0.0005 & $\mathrm{~L}$ & 84.0 & 0.001 & 0.001 & $\mathrm{H}$ \\
\hline 74.0 & 0.001 & 0.001 & $\mathrm{H}$ & 84.5 & 0.001 & 0.001 & $\mathrm{H}$ \\
\hline 74.4 & 0.0005 & 0.0005 & $\mathrm{~L}$ & 84.7498 & 0.001 & 0.001 & $\mathrm{~L}$ \\
\hline 74.5 & 0.001 & 0.001 & $\mathrm{H}$ & 85.0 & 0.001 & 0.001 & $\mathrm{H}$ \\
\hline 75.0 & 0.001 & 0.001 & $\mathrm{H}$ & 85.0 & 0.0005 & 0.0005 & $\mathrm{~L}$ \\
\hline 75.5 & 0.001 & 0.001 & $\mathrm{H}$ & 85.5 & 0.001 & 0.001 & $\mathrm{H}$ \\
\hline 76.0 & 0.001 & 0.001 & $\mathrm{H}$ & 85.5 & 0.0005 & 0.0005 & $\mathrm{~L}$ \\
\hline 76.3 & 0.0005 & 0.0005 & $\mathrm{~L}$ & 85.749775 & 0.001 & 0.001 & $\mathrm{~L}$ \\
\hline 76.3235 & 0.001 & 0.001 & $\mathrm{H}$ & 86.0 & 0.001 & 0.001 & $\mathrm{H}$ \\
\hline 76.3235 & 0.001 & 0.001 & $\mathrm{H}$ & 86.5 & 0.001 & 0.001 & $\mathrm{H}$ \\
\hline 76.411925 & 0.001 & 0.001 & $\mathrm{H}$ & 86.74975 & 0.001 & 0.001 & $\mathrm{~L}$ \\
\hline 76.5 & 0.001 & 0.001 & $\mathrm{H}$ & 87.0 & 0.001 & 0.001 & $\mathrm{H}$ \\
\hline 76.50035 & 0.001 & 0.001 & $\mathrm{H}$ & 87.5 & 0.001 & 0.001 & $\mathrm{H}$ \\
\hline 76.588775 & 0.001 & 0.001 & $\mathrm{H}$ & 87.749725 & 0.001 & 0.001 & $\mathrm{~L}$ \\
\hline 76.6772 & 0.001 & 0.001 & $\mathrm{H}$ & 87.9 & 0.0005 & 0.0005 & $\mathrm{~L}$ \\
\hline 76.75 & 0.001 & 0.001 & $\mathrm{~L}$ & 88.0 & 0.0005 & 0.0005 & $\mathrm{H}$ \\
\hline 76.765625 & 0.001 & 0.001 & $\mathrm{H}$ & 88.0 & 0.001 & 0.001 & $\mathrm{H}$ \\
\hline 76.8 & 0.0005 & 0.0005 & $\mathrm{~L}$ & 88.4 & 0.0005 & 0.0005 & $\mathrm{~L}$ \\
\hline 76.85405 & 0.001 & 0.001 & $\mathrm{H}$ & 88.5 & 0.001 & 0.001 & $\mathrm{H}$ \\
\hline 76.942475 & 0.001 & 0.001 & $\mathrm{H}$ & 88.7497 & 0.001 & 0.001 & $\mathrm{~L}$ \\
\hline 77.0 & 0.001 & 0.001 & $\mathrm{H}$ & 89.0 & 0.001 & 0.001 & $\mathrm{H}$ \\
\hline 77.0309 & 0.001 & 0.001 & $\mathrm{H}$ & 89.5 & 0.001 & 0.001 & $\mathrm{H}$ \\
\hline 77.119325 & 0.001 & 0.001 & $\mathrm{H}$ & 89.749675 & 0.001 & 0.001 & $\mathrm{~L}$ \\
\hline 77.20775 & 0.001 & 0.001 & $\mathrm{H}$ & 90.0 & 0.001 & 0.001 & $\mathrm{H}$ \\
\hline 77.296175 & 0.001 & 0.001 & $\mathrm{H}$ & 90.3 & 0.0005 & 0.0005 & $\mathrm{~L}$ \\
\hline 77.3 & 0.0005 & 0.0005 & $\mathrm{~L}$ & 90.5 & 0.001 & 0.001 & $\mathrm{H}$ \\
\hline 77.3846 & 0.001 & 0.001 & $\mathrm{H}$ & 90.74965 & 0.001 & 0.001 & $\mathrm{~L}$ \\
\hline 77.473025 & 0.001 & 0.001 & $\mathrm{H}$ & 90.8 & 0.0005 & 0.0005 & $\mathrm{~L}$ \\
\hline 77.5 & 0.001 & 0.001 & $\mathrm{H}$ & 91.0 & 0.001 & 0.001 & $\mathrm{H}$ \\
\hline 77.56145 & 0.001 & 0.001 & $\mathrm{H}$ & 91.3 & 0.0005 & 0.0005 & $\mathrm{~L}$ \\
\hline 77.749975 & 0.001 & 0.001 & $\mathrm{~L}$ & 91.5 & 0.001 & 0.001 & $\mathrm{H}$ \\
\hline 78.0 & 0.001 & 0.001 & $\mathrm{H}$ & 91.749625 & 0.001 & 0.001 & $\mathrm{~L}$ \\
\hline
\end{tabular}

TABLE VII. (Continued) 
TABLE VII. (Continued)

\begin{tabular}{|c|c|c|c|}
\hline$\underline{f_{\mathrm{L}}(\mathrm{Hz})}$ & LFS $(\mathrm{Hz})$ & HFS (Hz) & IFO \\
\hline 92.0 & 0.001 & 0.001 & $\mathrm{H}$ \\
\hline 92.5 & 0.001 & 0.001 & $\mathrm{H}$ \\
\hline 92.7496 & 0.001 & 0.001 & $\mathrm{~L}$ \\
\hline 93.0 & 0.001 & 0.001 & $\mathrm{H}$ \\
\hline 93.5 & 0.001 & 0.001 & $\mathrm{H}$ \\
\hline 93.7 & 0.0005 & 0.0005 & $\mathrm{~L}$ \\
\hline 93.749575 & 0.001 & 0.001 & $\mathrm{~L}$ \\
\hline 94.0 & 0.001 & 0.001 & $\mathrm{H}$ \\
\hline 94.2 & 0.0005 & 0.0005 & $\mathrm{~L}$ \\
\hline 94.2381 & 0.003 & 0.003 & $\mathrm{H}$ \\
\hline 94.2447 & 0.003 & 0.003 & $\mathrm{H}$ \\
\hline 94.5 & 0.001 & 0.001 & $\mathrm{H}$ \\
\hline 94.74955 & 0.001 & 0.001 & $\mathrm{~L}$ \\
\hline 95.0 & 0.001 & 0.001 & $\mathrm{H}$ \\
\hline 95.5 & 0.001 & 0.001 & $\mathrm{H}$ \\
\hline 95.749525 & 0.001 & 0.001 & $\mathrm{~L}$ \\
\hline 95.88316 & 0.0064 & 0.0032 & $\mathrm{~L}$ \\
\hline 96.0 & 0.0005 & 0.0005 & $\mathrm{H}$ \\
\hline 96.0 & 0.001 & 0.001 & $\mathrm{H}$ \\
\hline 96.5 & 0.001 & 0.001 & $\mathrm{H}$ \\
\hline 96.6 & 0.0005 & 0.0005 & $\mathrm{~L}$ \\
\hline 96.7495 & 0.001 & 0.001 & $\mathrm{~L}$ \\
\hline 97.0 & 0.001 & 0.001 & $\mathrm{H}$ \\
\hline 97.1 & 0.0005 & 0.0005 & $\mathrm{~L}$ \\
\hline 97.5 & 0.001 & 0.001 & $\mathrm{H}$ \\
\hline 97.749475 & 0.001 & 0.001 & $\mathrm{~L}$ \\
\hline 98.0 & 0.001 & 0.001 & $\mathrm{H}$ \\
\hline 98.5 & 0.001 & 0.001 & $\mathrm{H}$ \\
\hline 98.74945 & 0.001 & 0.001 & $\mathrm{~L}$ \\
\hline 99.0 & 0.001 & 0.001 & $\mathrm{H}$ \\
\hline 99.0 & 0.0005 & 0.0005 & $\mathrm{~L}$ \\
\hline 99.5 & 0.001 & 0.001 & $\mathrm{H}$ \\
\hline 99.5 & 0.0005 & 0.0005 & $\mathrm{~L}$ \\
\hline 99.749425 & 0.001 & 0.001 & $\mathrm{~L}$ \\
\hline 99.9989 & 0.001 & 0.001 & $\mathrm{H}$ \\
\hline 100.0 & 0.001 & 0.001 & $\mathrm{H}$ \\
\hline
\end{tabular}

\section{50-mHz signal-frequency bands where the upper limit value does not hold}

See Table VII

TABLE VIII. 50-mHz search-frequency bands that are excluded from the results. Bands are excluded from the results if they were identified as disturbed based on visual inspection (D), if they were identified as mildly disturbed based on visual inspection then excluded later in the analysys (M), if they contained a hardware injection (I), or where the results were produced from entirely fake data as detailed in Table I (C). Bands labeled D, C, or I are excluded from the analysis.

\begin{tabular}{lccccccc}
\hline \hline $\begin{array}{c}\text { Start } \\
\text { band }\end{array}$ & $\begin{array}{c}\text { Band } \\
\text { type }\end{array}$ & $\begin{array}{c}\text { Start } \\
\text { band }\end{array}$ & $\begin{array}{c}\text { Band } \\
\text { type }\end{array}$ & $\begin{array}{c}\text { Start } \\
\text { band }\end{array}$ & $\begin{array}{c}\text { Band } \\
\text { type }\end{array}$ & $\begin{array}{c}\text { Start } \\
\text { band }\end{array}$ & $\begin{array}{c}\text { Band } \\
\text { type }\end{array}$ \\
\hline 20.40 & $\mathrm{M}$ & 20.90 & $\mathrm{D}$ & 20.80 & $\mathrm{M}$ & 21.45 & $\mathrm{M}$ \\
22.40 & $\mathrm{D}$ & 23.90 & $\mathrm{M}$ & 24.45 & $\mathrm{D}$ & 24.20 & $\mathrm{M}$ \\
25.25 & $\mathrm{M}$ & 25.60 & $\mathrm{M}$ & 26.05 & $\mathrm{M}$ & 26.90 & $\mathrm{M}$ \\
27.50 & $\mathrm{D}$ & 27.45 & $\mathrm{M}$ & 27.85 & $\mathrm{D}$ & 27.55 & $\mathrm{M}$ \\
28.55 & $\mathrm{D}$ & 28.90 & $\mathrm{M}$ & 29.15 & $\mathrm{D}$ & 30.60 & $\mathrm{D}$ \\
30.85 & $\mathrm{M}$ & 31.10 & $\mathrm{M}$ & 31.40 & $\mathrm{I}$ & 31.75 & $\mathrm{M}$ \\
32.35 & $\mathrm{M}$ & 32.90 & $\mathrm{M}$ & 33.05 & $\mathrm{M}$ & 34.80 & $\mathrm{M}$ \\
34.60 & $\mathrm{C}$ & 34.65 & $\mathrm{C}$ & 34.70 & $\mathrm{C}$ & 34.75 & $\mathrm{C}$ \\
35.20 & $\mathrm{C}$ & 35.25 & $\mathrm{C}$ & 35.30 & $\mathrm{C}$ & 35.35 & $\mathrm{C}$ \\
35.70 & $\mathrm{M}$ & 35.80 & $\mathrm{C}$ & 35.85 & $\mathrm{C}$ & 35.90 & $\mathrm{C}$ \\
35.95 & $\mathrm{C}$ & 36.60 & $\mathrm{M}$ & 36.60 & $\mathrm{C}$ & 36.65 & $\mathrm{C}$ \\
36.70 & $\mathrm{C}$ & 36.75 & $\mathrm{C}$ & 37.25 & $\mathrm{M}$ & 39.75 & $\mathrm{M}$ \\
40.20 & $\mathrm{M}$ & 40.85 & $\mathrm{D}$ & 42.80 & $\mathrm{M}$ & 43.65 & $\mathrm{M}$ \\
44.70 & $\mathrm{D}$ & 44.65 & $\mathrm{M}$ & 45.30 & $\mathrm{D}$ & 45.35 & $\mathrm{M}$ \\
46.90 & $\mathrm{M}$ & 47.65 & $\mathrm{M}$ & 48.95 & $\mathrm{M}$ & 50.25 & $\mathrm{M}$ \\
51.00 & $\mathrm{M}$ & 52.30 & $\mathrm{M}$ & 52.60 & $\mathrm{M}$ & 52.80 & $\mathrm{I}$ \\
53.05 & $\mathrm{M}$ & 54.70 & $\mathrm{M}$ & 55.05 & $\mathrm{M}$ & 55.60 & $\mathrm{M}$ \\
56.80 & $\mathrm{D}$ & 57.10 & $\mathrm{M}$ & 58.95 & $\mathrm{M}$ & 59.50 & $\mathrm{M}$ \\
59.55 & $\mathrm{M}$ & 59.95 & $\mathrm{C}$ & 60.00 & $\mathrm{C}$ & 61.00 & $\mathrm{M}$ \\
61.05 & $\mathrm{M}$ & 62.45 & $\mathrm{D}$ & 62.05 & $\mathrm{M}$ & 66.65 & $\mathrm{M}$ \\
74.50 & $\mathrm{D}$ & 74.45 & $\mathrm{M}$ & 75.00 & $\mathrm{M}$ & 76.60 & $\mathrm{D}$ \\
76.65 & $\mathrm{M}$ & 83.30 & $\mathrm{M}$ & 85.80 & $\mathrm{M}$ & 89.40 & $\mathrm{D}$ \\
89.35 & $\mathrm{M}$ & 90.00 & $\mathrm{M}$ & 99.95 & $\mathrm{D}$ & & \\
\hline \hline
\end{tabular}

[1] https://www.einsteinathome.org/.

[2] M. A. Papa et al., Hierarchical follow-up of subthreshold candidates of an all-sky Einstein@Home search for continuous gravitational waves on LIGO sixth science run data, Phys. Rev. D 94, 122006 (2016).

[3] B. P. Abbott et al. (LIGO Scientific Collaboration and Virgo Collaboration), Results of the deepest all-sky survey for continuous gravitational waves on LIGO S6 data running on the Einstein@Home volunteer distributed computing project, Phys. Rev. D 94, 102002 (2016).

[4] B.P. Abbott et al. (LIGO Scientific Collaboration and VIRGO Collaboration), Comprehensive all-sky search for periodic gravitational waves in the sixth science run LIGO data, Phys. Rev. D 94, 042002 (2016).

[5] A. Singh, M. A. Papa, H.-B. Eggenstein, S. Zhu, H. Pletsch, B. Allen, O. Bock, B. Maschenchalk, R. Prix, and X. Siemens, Results of an all-sky high-frequency Einstein@Home search for continuous gravitational waves in LIGO 5th science run, Phys. Rev. D 94, 064061 (2016).

[6] J. Aasi et al. (LIGO Scientific Collaboration and VIRGO Collaboration), First low frequency all-sky search for continuous gravitational wave signals, Phys. Rev. D 93, 042007 (2016). 
[7] B. P. Abbott et al. (LIGO Scientific Collaboration), Einstein@Home all-sky search for periodic gravitational waves in LIGO S5 data, Phys. Rev. D 87, 042001 (2013).

[8] B. P. Abbott et al. (LIGO Scientific Collaboration and VIRGO Collaboration), All-sky Search for Periodic Gravitational Waves in the Full S5 Data, Phys. Rev. D 85, 022001 (2012).

[9] B. P. Abbott et al. (LIGO Scientific Collaboration), Einstein@Home search for periodic gravitational waves in early S5 LIGO data, Phys. Rev. D 80, 042003 (2009).

[10] B. P. Abbott et al. (LIGO Scientific Collaboration), All-sky LIGO Search for Periodic Gravitational Waves in the Early S5 Data, Phys. Rev. Lett. 102, 111102 (2009).

[11] J. Aasi et al. (LIGO Scientific Collaboration and Virgo Collaboration), Application of a Hough search for continuous gravitational waves on data from the 5th LIGO science run, Classical Quantum Gravity 31, 085014 (2014).

[12] J. Aasi et al. (LIGO Scientific Collaboration and Virgo Collaboration), Implementation of an $\mathcal{F}$-statistic all-sky search for continuous gravitational waves in Virgo VSR1 data, Classical Quantum Gravity 31, 165014 (2014).

[13] B. P. Abbott et al. (LIGO Scientific Collaboration), All-sky search for periodic gravitational waves in LIGO S4 data, Phys. Rev. D 77, 022001 (2008).

[14] B. P. Abbott et al. (LIGO Scientific Collaboration), Einstein@Home search for periodic gravitational waves in LIGO S4 data, Phys. Rev. D 79, 022001 (2009).

[15] B. Abbott et al. (LIGO Scientific Collaboration), Searches for periodic gravitational waves from unknown isolated sources and Scorpius X-1: Results from the second LIGO science run, Phys. Rev. D 76, 082001 (2007).

[16] LIGO Scientific Collaboration, LIGO: The Laser Interferometer Gravitational-Wave Observatory, Rep. Prog. Phys. 72, 076901 (2009).

[17] B. Abbott et al. (LIGO Scientific Collaboration), GW150914: The Advanced LIGO Detectors in the Era of First Discoveries, Phys. Rev. Lett. 116, 131103 (2016).

[18] J. Aasi et al. (LIGO Scientific Collaboration), Advanced LIGO, Classical Quantum Gravity 32, 074001 (2015).

[19] J. Abadie et al. (LIGO Scientific Collaboration and Virgo Collaboration), Sensitivity achieved by the LIGO and Virgo gravitational wave detectors during LIGO's sixth and Virgo's second and third science runs, arXiv:1203.2674.

[20] H. J. Pletsch and B. Allen, Exploiting Large-Scale Correlations to Detect Continuous Gravitational Waves, Phys. Rev. Lett. 103, 181102 (2009).

[21] H. J. Pletsch, Parameter-space correlations of the optimal statistic for continuous gravitational-wave detection, Phys. Rev. D 78, 102005 (2008).

[22] H. J. Pletsch, Parameter-space metric of semicoherent searches for continuous gravitational waves, Phys. Rev. D 82, 042002 (2010).
[23] C. Cutler and B.F. Schutz, The generalized $\mathcal{F}$-statistic: multiple detectors and multiple gravitational wave pulsars, Phys. Rev. D 72, 063006 (2005).

[24] B. Krishnan, A. M. Sintes, M. A. Papa, B. F. Schutz, S. Frasca, and C. Palomba, The Hough transform search for continuous gravitational waves, Phys. Rev. D 70, 082001 (2004).

[25] P. Astone, A. Colla, S. D'Antonio, S. Frasca, and C. Palomba, Method for all-sky searches of continuous gravitational wave signals using the frequency-Hough transform, Phys. Rev. D 90, 042002 (2014).

[26] http://boinc.berkeley.edu/.

[27] D. P. Anderson, BOINC: A System for Public-Resource Computing and Storage, in Proceedings of the Fifth IEEE/ACM International Workshop on Grid Computing (GRID04), pp. 4-10 (2004).

[28] D. P. Anderson, C. Christensen, and B. Allen, Designing a Runtime System for Volunteer Computing, in Proceedings of the 2006 ACM/IEEE conference on Supercomputing, pp. 126-136 (2006).

[29] D. Keitel, Robust semicoherent searches for continuous gravitational waves with noise and signal models including hours to days long transients, Phys. Rev. D 93, 084024 (2016).

[30] D. Keitel, R. Prix, M. A. Papa, P. Leaci, and M. Siddiqi, Search for continuous gravitational waves: Improving robustness versus instrumental artifacts, Phys. Rev. D 89, 064023 (2014).

[31] S. J. Zhu et al., An Einstein@Home search for continuous gravitational waves from Cassiopeia A, Phys. Rev. D 94, 082008 (2016).

[32] C. Biwer et al., Validating gravitational-wave detections: The Advanced LIGO hardware injection system, Phys. Rev. D 95, 062002 (2017).

[33] J. Aasi et al. (LIGO Scientific Collaboration and VIRGO Collaboration), Directed search for continuous gravitational waves from the Galactic center, Phys. Rev. D 88, 102002 (2013).

[34] A. Singh, M. A. Papa, H.-B. Eggenstein, and S. Walsh, Adaptive clustering algorithm for continuous gravitational wave searches, arXiv:1707.02676.

[35] http://www.aei.mpg.de/24838/02 Computing and ATLAS.

[36] S. J. Zhu, M. A. Papa, and S. Walsh, preceding article, New veto for continuous gravitational wave searches, Phys. Rev. D 96, 124007 (2017).

[37] B. Behnke, M. A. Papa, and R. Prix, Postprocessing methods used in the search for continuous gravitationalwave signals from the Galactic Center, Phys. Rev. D 91, 064007 (2015).

[38] J. Aasi et al. (LIGO Scientific Collaboration and VIRGO Collaboration), All-sky search for periodic gravitational waves in the O1 LIGO Data, arXiv:1707.02667.

[39] J. Ming, B. Krishnan, M. A. Papa, C. Aulbert, and H. Fehrmann, Optimal directed searches for continuous gravitational waves, Phys. Rev. D 93, 064011 (2016).

B. P. Abbott,,${ }^{1}$ R. Abbott,,${ }^{1}$ T. D. Abbott,${ }^{2}$ F. Acernese,${ }^{3,4}$ K. Ackley, ${ }^{5}$ C. Adams,${ }^{6}$ T. Adams, ${ }^{7}$ P. Addesso, ${ }^{8}$ R. X. Adhikari, ${ }^{1}$ V. B. Adya, ${ }^{9}$ C. Affeldt, ${ }^{9}$ M. Afrough, ${ }^{10}$ B. Agarwal, ${ }^{11}$ K. Agatsuma, ${ }^{12}$ N. Aggarwal, ${ }^{13}$ O. D. Aguiar, ${ }^{14}$ L. Aiello, ${ }^{15,16}$ 
A. Ain, ${ }^{17}$ B. Allen, ${ }^{9,18,19}$ G. Allen, ${ }^{11}$ A. Allocca, ${ }^{20,21}$ P. A. Altin, ${ }^{22}$ A. Amato, ${ }^{23}$ A. Ananyeva, ${ }^{1}$ S. B. Anderson, ${ }^{1}$ W. G. Anderson, ${ }^{18}$ S. Antier, ${ }^{24}$ S. Appert, ${ }^{1}$ K. Arai, ${ }^{1}$ M. C. Araya, ${ }^{1}$ J. S. Areeda, ${ }^{25}$ N. Arnaud, ${ }^{24,26}$ S. Ascenzi, ${ }^{27,16}$ G. Ashton, ${ }^{9}$ M. Ast,${ }^{28}$ S. M. Aston, ${ }^{6}$ P. Astone, ${ }^{29}$ P. Aufmuth, ${ }^{19}$ C. Aulbert,${ }^{9}$ K. AultONeal,${ }^{30}$ A. Avila-Alvarez, ${ }^{25}$ S. Babak, ${ }^{31}$ P. Bacon, ${ }^{32}$ M. K. M. Bader, ${ }^{12}$ S. Bae, ${ }^{33}$ P. T. Baker,${ }^{34,35}$ F. Baldaccini, ${ }^{36,37}$ G. Ballardin, ${ }^{26}$ S. W. Ballmer, ${ }^{38}$ S. Banagiri, ${ }^{39}$ J. C. Barayoga, ${ }^{1}$ S. E. Barclay, ${ }^{40}$ B. C. Barish,${ }^{1}$ D. Barker,${ }^{41}$ F. Barone,${ }^{3,4}$ B. Barr, ${ }^{40}$ L. Barsotti, ${ }^{13}$ M. Barsuglia, ${ }^{32}$ D. Barta, ${ }^{42}$ J. Bartlett, ${ }^{41}$ I. Bartos ${ }^{43}$ R. Bassiri, ${ }^{44}$ A. Basti ${ }^{20,21}$ J. C. Batch, ${ }^{41}$ C. Baune, ${ }^{9}$ M. Bawaj,${ }^{45,37}$ M. Bazzan,${ }^{46,47}$ B. Bécsy, ${ }^{48}$ C. Beer, ${ }^{9}$ M. Bejger, ${ }^{49}$ I. Belahcene, ${ }^{24}$ A. S. Bell, ${ }^{40}$ B. K. Berger, ${ }^{1}$ G. Bergmann, ${ }^{9}$ C. P. L. Berry ${ }^{50}$ D. Bersanetti, ${ }^{51,52}$ A. Bertolini, ${ }^{12}$ J. Betzwieser, ${ }^{6}$ S. Bhagwat,${ }^{38}$ R. Bhandare,${ }^{53}$ I. A. Bilenko, ${ }^{54}$ G. Billingsley, ${ }^{1}$ C. R. Billman, ${ }^{5}$ J. Birch, ${ }^{6}$ R. Birney,${ }^{55}$ O. Birnholtz, ${ }^{9}$ S. Biscans, ${ }^{13}$ A. Bisht,${ }^{19}$ M. Bitossi,${ }^{26,21}$ C. Biwer,${ }^{38}$ M. A. Bizouard ${ }^{24}$ J. K. Blackburn, ${ }^{1}$ J. Blackman, ${ }^{56}$ C. D. Blair, ${ }^{57}$ D. G. Blair, ${ }^{57}$ R. M. Blair ${ }^{41}$ S. Bloemen, ${ }^{58}$ O. Bock, ${ }^{9}$ N. Bode, ${ }^{9}$ M. Boer, ${ }^{59}$ G. Bogaert, ${ }^{59}$ A. Bohe, ${ }^{31}$ F. Bondu, ${ }^{60}$ R. Bonnand, ${ }^{7}$ B. A. Boom, ${ }^{12}$ R. Bork, ${ }^{1}$ V. Boschi, ${ }^{20,21}$ S. Bose ${ }^{61,17}$ Y. Bouffanais,${ }^{32}$ A. Bozzi,${ }^{26}$ C. Bradaschia, ${ }^{21}$ P. R. Brady, ${ }^{18}$ V. B. Braginsky,${ }^{\dagger, 54}$ M. Branchesi, ${ }^{62,63}$ J. E. Brau, ${ }^{64}$ T. Briant, ${ }^{65}$ A. Brillet, ${ }^{59}$ M. Brinkmann, ${ }^{9}$ V. Brisson, ${ }^{24}$ P. Brockill, ${ }^{18}$ J. E. Broida ${ }^{66}$ A. F. Brooks, ${ }^{1}$ D. A. Brown, ${ }^{38}$ D. D. Brown, ${ }^{50}$ N. M. Brown, ${ }^{13}$ S. Brunett, ${ }^{1}$ C. C. Buchanan, ${ }^{2}$ A. Buikema, ${ }^{13}$ T. Bulik, ${ }^{67}$ H. J. Bulten, ${ }^{68,12}$ A. Buonanno, ${ }^{31,69}$ D. Buskulic, ${ }^{7}$ C. Buy,${ }^{32}$ R. L. Byer, ${ }^{44}$ M. Cabero, ${ }^{9}$ L. Cadonati, ${ }^{70}$ G. Cagnoli, ${ }^{23,71}$ C. Cahillane, ${ }^{1}$ J. Calderón Bustillo, ${ }^{70}$ T. A. Callister, ${ }^{1}$ E. Calloni, ${ }^{72,4}$ J. B. Camp,${ }^{73}$ P. Canizares, ${ }^{58}$ K. C. Cannon,${ }^{74}$ H. Cao, ${ }^{75}$ J. Cao, ${ }^{76}$ C. D. Capano, ${ }^{9}$ E. Capocasa, ${ }^{32}$ F. Carbognani, ${ }^{26}$ S. Caride, ${ }^{77}$ M. F. Carney, ${ }^{78}$ J. Casanueva Diaz, ${ }^{24}$ C. Casentini,${ }^{27,16}$ S. Caudill, ${ }^{18}$ M. Cavaglià, ${ }^{10}$ F. Cavalier ${ }^{24}$ R. Cavalieri, ${ }^{26}$ G. Cella,${ }^{21}$ C. B. Cepeda,${ }^{1}$ L. Cerboni Baiardi,${ }^{62,63}$ G. Cerretani, ${ }^{20,21}$ E. Cesarini, ${ }^{27,16}$ S. J. Chamberlin, ${ }^{79}$ M. Chan, ${ }^{40}$ S. Chao, ${ }^{80}$ P. Charlton, ${ }^{81}$ E. Chassande-Mottin, ${ }^{32}$ D. Chatterjee, ${ }^{18}$ B. D. Cheeseboro, ${ }^{34,35}$ H. Y. Chen,${ }^{82}$ Y. Chen, ${ }^{56}$ H.-P. Cheng, ${ }^{5}$ A. Chincarini,${ }^{52}$ A. Chiummo, ${ }^{26}$ T. Chmiel,${ }^{78}$ H. S. Cho, ${ }^{83}$ M. Cho, ${ }^{69}$ J. H. Chow, ${ }^{22}$ N. Christensen, ${ }^{66,59}$ Q. Chu, ${ }^{57}$ A. J. K. Chua,${ }^{84}$ S. Chua,${ }^{65}$ A. K. W. Chung, ${ }^{85}$ S. Chung, ${ }^{57}$ G. Ciani, ${ }^{5}$

R. Ciolfi, ${ }^{86,87}$ C. E. Cirelli, ${ }^{44}$ A. Cirone,${ }^{51,52}$ F. Clara,${ }^{41}$ J. A. Clark,${ }^{70}$ F. Cleva, ${ }^{59}$ C. Cocchieri, ${ }^{10}$ E. Coccia,${ }^{15,16}$ P.-F. Cohadon, ${ }^{65}$ A. Colla, ${ }^{88,29}$ C. G. Collette, ${ }^{89}$ L. R. Cominsky, ${ }^{90}$ M. Constancio Jr., ${ }^{14}$ L. Conti, ${ }^{47}$ S. J. Cooper, ${ }^{50}$ P. Corban, ${ }^{6}$ T. R. Corbitt, ${ }^{2}$ K. R. Corley, ${ }^{43}$ N. Cornish, ${ }^{91}$ A. Corsi,${ }^{77}$ S. Cortese,${ }^{26}$ C. A. Costa, ${ }^{14}$ M. W. Coughlin, ${ }^{66}$ S. B. Coughlin, ${ }^{92,93}$ J.-P. Coulon, ${ }^{59}$ S. T. Countryman, ${ }^{43}$ P. Couvares, ${ }^{1}$ P. B. Covas, ${ }^{94}$ E. E. Cowan, ${ }^{70}$ D. M. Coward ${ }^{57}$ M. J. Cowart, ${ }^{6}$ D. C. Coyne, ${ }^{1}$ R. Coyne, ${ }^{77}$ J. D. E. Creighton, ${ }^{18}$ T. D. Creighton,${ }^{95}$ J. Cripe, ${ }^{2}$ S. G. Crowder, ${ }^{96}$ T. J. Cullen, ${ }^{25}$ A. Cumming ${ }^{40}$ L. Cunningham ${ }^{40}$ E. Cuoco, ${ }^{26}$ T. Dal Canton, ${ }^{73}$ S. L. Danilishin, ${ }^{19,9}$ S. D’ Antonio, ${ }^{16}$ K. Danzmann, ${ }^{19,9}$ A. Dasgupta, ${ }^{97}$ C. F. Da Silva Costa,${ }^{5}$ V. Dattilo, ${ }^{26}$ I. Dave,${ }^{53}$ M. Davier,${ }^{24}$ D. Davis, ${ }^{38}$ E. J. Daw,${ }^{98}$ B. Day, ${ }^{70}$ S. De,${ }^{38}$ D. DeBra ${ }^{44}$ E. Deelman, ${ }^{99}$ J. Degallaix,${ }^{23}$ M. De Laurentis ${ }^{72,4}$ S. Deléglise, ${ }^{65}$ W. Del Pozzo, ${ }^{50,20,21}$ T. Denker, ${ }^{9}$ T. Dent, ${ }^{9}$ V. Dergachev, ${ }^{31}$ R. De Rosa,${ }^{72,4}$ R. T. DeRosa,${ }^{6}$ R. DeSalvo, ${ }^{100}$ J. Devenson, ${ }^{55}$ R. C. Devine, ${ }^{34,35}$ S. Dhurandhar, ${ }^{17}$ M. C. Díaz ${ }^{95}$ L. Di Fiore, ${ }^{4}$ M. Di Giovanni, ${ }^{101,87}$ T. Di Girolamo, ${ }^{72,4,43}$ A. Di Lieto, ${ }^{20,21}$ S. Di Pace, ${ }^{88,29}$ I. Di Palma, ${ }^{88,29}$ F. Di Renzo, ${ }^{20,21}$ Z. Doctor, ${ }^{82}$ V. Dolique, ${ }^{23}$ F. Donovan, ${ }^{13}$ K. L. Dooley, ${ }^{10}$ S. Doravari, ${ }^{9}$ I. Dorrington, ${ }^{93}$ R. Douglas, ${ }^{40}$ M. Dovale Álvarez ${ }^{50}$ T. P. Downes, ${ }^{18}$ M. Drago, ${ }^{9}$ R. W. P. Drever, ${ }^{\dagger, 1}$ J. C. Driggers, ${ }^{41}$ Z. Du, ${ }^{76}$ M. Ducrot, ${ }^{7}$ J. Duncan, ${ }^{92}$ S. E. Dwyer, ${ }^{41}$ T. B. Edo, ${ }^{98}$ M. C. Edwards, ${ }^{66}$ A. Effler ${ }^{6}$ H.-B. Eggenstein, ${ }^{9}$ P. Ehrens, ${ }^{1}$ J. Eichholz,,${ }^{1}$ S. S. Eikenberry, ${ }^{5}$

R. A. Eisenstein, ${ }^{13}$ R. C. Essick, ${ }^{13}$ Z. B. Etienne, ${ }^{34,35}$ T. Etzel, ${ }^{1}$ M. Evans, ${ }^{13}$ T. M. Evans, ${ }^{6}$ M. Factourovich,${ }^{43}$ V. Fafone, ${ }^{27,16,15}$ H. Fair, ${ }^{38}$ S. Fairhurst,${ }^{93}$ X. Fan, ${ }^{76}$ S. Farinon, ${ }^{52}$ B. Farr, ${ }^{82}$ W. M. Farr, ${ }^{50}$ E. J. Fauchon-Jones, ${ }^{93}$ M. Favata, ${ }^{102}$ M. Fays ${ }^{93}$ H. Fehrmann, ${ }^{9}$ J. Feicht, ${ }^{1}$ M. M. Fejer, ${ }^{44}$ A. Fernandez-Galiana, ${ }^{13}$ I. Ferrante,${ }^{20,21}$ E. C. Ferreira,${ }^{14}$ F. Ferrini, ${ }^{26}$ F. Fidecaro, ${ }^{20,21}$ I. Fiori, ${ }^{26}$ D. Fiorucci, ${ }^{32}$ R. P. Fisher,${ }^{38}$ R. Flaminio, ${ }^{23,103}$ M. Fletcher, ${ }^{40}$ H. Fong, ${ }^{104}$ P. W. F. Forsyth, ${ }^{22}$ S. S. Forsyth, ${ }^{70}$ J.-D. Fournier, ${ }^{59}$ S. Frasca, ${ }^{88,29}$ F. Frasconi, ${ }^{21}$ Z. Frei, ${ }^{48}$ A. Freise,${ }^{50}$ R. Frey, ${ }^{64}$ V. Frey,${ }^{24}$ E. M. Fries, ${ }^{1}$ P. Fritschel, ${ }^{13}$ V. V. Frolov, ${ }^{6}$ P. Fulda,${ }^{5,73}$ M. Fyffe, ${ }^{6}$ H. Gabbard, ${ }^{9}$ M. Gabel,${ }^{105}$ B. U. Gadre,${ }^{17}$ S. M. Gaebel,${ }^{50}$ J. R. Gair, ${ }^{106}$ L. Gammaitoni, ${ }^{36}$ M. R. Ganija, ${ }^{75}$ S. G. Gaonkar, ${ }^{17}$ F. Garufi, ${ }^{72,4}$ S. Gaudio, ${ }^{30}$ G. Gaur, ${ }^{107}$ V. Gayathri, ${ }^{108}$ N. Gehrels,, 73 G. Gemme, ${ }^{52}$ E. Genin, ${ }^{26}$ A. Gennai ${ }^{21}$ D. George,${ }^{11}$ J. George ${ }^{53}$ L. Gergely, ${ }^{109}$ V. Germain, ${ }^{7}$ S. Ghonge,${ }^{70}$ Abhirup Ghosh, ${ }^{110}$ Archisman Ghosh, ${ }^{110,12}$ S. Ghosh, ${ }^{58,12}$ J. A. Giaime, ${ }^{2,6}$ K. D. Giardina, ${ }^{6}$ A. Giazotto, ${ }^{21}$ K. Gill, ${ }^{30}$

L. Glover, ${ }^{100}$ E. Goetz, ${ }^{9}$ R. Goetz,${ }^{5}$ S. Gomes, ${ }^{93}$ G. González, ${ }^{2}$ J. M. Gonzalez Castro, ${ }^{20,21}$ A. Gopakumar, ${ }^{111}$ M. L. Gorodetsky ${ }^{54}$ S. E. Gossan, ${ }^{1}$ M. Gosselin, ${ }^{26}$ R. Gouaty, ${ }^{7}$ A. Grado,${ }^{12,4}$ C. Graef, ${ }^{40}$ M. Granata, ${ }^{23}$ A. Grant, ${ }^{40}$ S. Gras, ${ }^{13}$ C. Gray, ${ }^{41}$ G. Greco, ${ }^{62,63}$ A. C. Green,${ }^{50}$ P. Groot ${ }^{58}$ H. Grote, ${ }^{9}$ S. Grunewald, ${ }^{31}$ P. Gruning, ${ }^{24}$ G. M. Guidi,,${ }^{62,63}$ X. Guo, ${ }^{76}$ A. Gupta, ${ }^{79}$ M. K. Gupta, ${ }^{97}$ K. E. Gushwa, ${ }^{1}$ E. K. Gustafson, ${ }^{1}$ R. Gustafson, ${ }^{113}$ B. R. Hall, ${ }^{61}$ E. D. Hall, ${ }^{1}$ G. Hammond ${ }^{40}$ M. Haney, ${ }^{111}$ M. M. Hanke, ${ }^{9}$ J. Hanks, ${ }^{41}$ C. Hanna,${ }^{79}$ O. A. Hannuksela ${ }^{85}$ J. Hanson, ${ }^{6}$ T. Hardwick, ${ }^{2}$ J. Harms, ${ }^{62,63}$ G. M. Harry, ${ }^{114}$ I. W. Harry, ${ }^{31}$ M. J. Hart, ${ }^{40}$ C.-J. Haster, ${ }^{104}$ K. Haughian, ${ }^{40}$ J. Healy, ${ }^{115}$ A. Heidmann, ${ }^{65}$ 
M. C. Heintze ${ }^{6}$ H. Heitmann, ${ }^{59}$ P. Hello, ${ }^{24}$ G. Hemming, ${ }^{26}$ M. Hendry, ${ }^{40}$ I. S. Heng, ${ }^{40}$ J. Hennig, ${ }^{40}$ J. Henry, ${ }^{115}$ A. W. Heptonstall, ${ }^{1}$ M. Heurs, ${ }^{9,19}$ S. Hild, ${ }^{40}$ D. Hoak,${ }^{26}$ D. Hofman, ${ }^{23}$ K. Holt,${ }^{6}$ D. E. Holz,${ }^{82}$ P. Hopkins,${ }^{93}$ C. Horst,${ }^{18}$ J. Hough, ${ }^{40}$ E. A. Houston, ${ }^{40}$ E. J. Howell,${ }^{57}$ Y. M. Hu, ${ }^{9}$ E. A. Huerta,${ }^{11}$ D. Huet,${ }^{24}$ B. Hughey, ${ }^{30}$ S. Husa,${ }^{94}$ S. H. Huttner, ${ }^{40}$

T. Huynh-Dinh, ${ }^{6}$ N. Indik, ${ }^{9}$ D. R. Ingram, ${ }^{41}$ R. Inta, ${ }^{77}$ G. Intini, ${ }^{88,29}$ H. N. Isa, ${ }^{40}$ J.-M. Isac, ${ }^{65}$ M. Isi, ${ }^{1}$ B. R. Iyer, ${ }^{10}$ K. Izumi, ${ }^{41}$ T. Jacqmin,${ }^{65}$ K. Jani, ${ }^{70}$ P. Jaranowski, ${ }^{116}$ S. Jawahar, ${ }^{117}$ F. Jiménez-Forteza, ${ }^{94}$ W. W. Johnson, ${ }^{2}$ D. I. Jones, ${ }^{118}$

R. Jones, ${ }^{40}$ R. J. G. Jonker, ${ }^{12}$ L. Ju,${ }^{57}$ J. Junker, ${ }^{9}$ C. V. Kalaghatgi,${ }^{93}$ V. Kalogera, ${ }^{92}$ S. Kandhasamy, ${ }^{6}$ G. Kang, ${ }^{33}$ J. B. Kanner, ${ }^{1}$ S. Karki,${ }^{64}$ K. S. Karvinen, ${ }^{9}$ M. Kasprzack, ${ }^{2}$ M. Katolik, ${ }^{11}$ E. Katsavounidis ${ }^{13}$ W. Katzman, ${ }^{6}$ S. Kaufer, ${ }^{19}$ K. Kawabe ${ }^{41}$ F. Kéfélian, ${ }^{59}$ D. Keitel,${ }^{40}$ A. J. Kemball, ${ }^{11}$ R. Kennedy, ${ }^{98}$ C. Kent, ${ }^{93}$ J. S. Key, ${ }^{119}$ F. Y. Khalili, ${ }^{54}$ I. Khan,,${ }^{15,16}$ S. Khan, ${ }^{9}$ Z. Khan, ${ }^{97}$ E. A. Khazanov, ${ }^{120}$ N. Kijbunchoo, ${ }^{41}$ Chunglee Kim, ${ }^{121}$ J. C. Kim, ${ }^{122}$ W. Kim, ${ }^{75}$ W. S. Kim, ${ }^{123}$ Y.-M. Kim, ${ }^{83,121}$ S. J. Kimbrell, ${ }^{70}$ E. J. King, ${ }^{75}$ P. J. King, ${ }^{41}$ R. Kirchhoff, ${ }^{9}$ J. S. Kissel, ${ }^{41}$ L. Kleybolte, ${ }^{28}$ S. Klimenko, ${ }^{5}$ P. Koch, ${ }^{9}$ S. M. Koehlenbeck, ${ }^{9}$ S. Koley, ${ }^{12}$ V. Kondrashov, ${ }^{1}$ A. Kontos,${ }^{13}$ M. Korobko, ${ }^{28}$ W. Z. Korth, ${ }^{1}$ I. Kowalska, ${ }^{67}$ D. B. Kozak, ${ }^{1}$ C. Krämer, ${ }^{9}$ V. Kringel, ${ }^{9}$ B. Krishnan, ${ }^{9}$ A. Królak, ${ }^{124,125}$ G. Kuehn, ${ }^{9}$ P. Kumar, ${ }^{104}$ R. Kumar, ${ }^{97}$ S. Kumar, ${ }^{110}$ L. Kuo ${ }^{80}$ A. Kutynia, ${ }^{124}$ S. Kwang, ${ }^{18}$ B. D. Lackey, ${ }^{31}$ K. H. Lai ${ }^{85}$ M. Landry, ${ }^{41}$ R. N. Lang, ${ }^{18}$ J. Lange, ${ }^{115}$ B. Lantz,${ }^{44}$ R. K. Lanza, ${ }^{13}$ A. Lartaux-Vollard, ${ }^{24}$ P. D. Lasky, ${ }^{126}$ M. Laxen, ${ }^{6}$ A. Lazzarini, ${ }^{1}$ C. Lazzaro, ${ }^{47}$ P. Leaci, ${ }^{88,29}$ S. Leavey, ${ }^{40}$ C. H. Lee, ${ }^{83}$ H. K. Lee, ${ }^{127}$ H. M. Lee, ${ }^{121}$ H. W. Lee, ${ }^{122}$ K. Lee, ${ }^{40}$ J. Lehmann,${ }^{9}$ A. Lenon, ${ }^{34,35}$ M. Leonardi, ${ }^{101,87}$ N. Leroy, ${ }^{24}$ N. Letendre, ${ }^{7}$ Y. Levin, ${ }^{126}$ T. G. F. Li ${ }^{85}$ A. Libson, ${ }^{13}$ T. B. Littenberg, ${ }^{128}$ J. Liu, ${ }^{57}$ R. K. L. Lo, ${ }^{85}$ N. A. Lockerbie, ${ }^{117}$ L. T. London, ${ }^{93}$ J. E. Lord, ${ }^{38}$ M. Lorenzini, ${ }^{15,16}$ V. Loriette, ${ }^{129}$ M. Lormand, ${ }^{6}$ G. Losurdo, ${ }^{21}$ J. D. Lough,${ }^{9,19}$ G. Lovelace ${ }^{25}$ H. Lück, ${ }^{19,9}$ D. Lumaca, ${ }^{27,16}$ A. P. Lundgren, ${ }^{9}$ R. Lynch, ${ }^{13}$ Y. Ma, ${ }^{56}$ S. Macfoy,${ }^{55}$ B. Machenschalk, ${ }^{9}$ M. MacInnis, ${ }^{13}$ D. M. Macleod, ${ }^{2}$ I. Magaña Hernandez, ${ }^{85}$ F. Magaña-Sandoval,${ }^{38}$ L. Magaña Zertuche, ${ }^{38}$ R. M. Magee, ${ }^{79}$ E. Majorana, ${ }^{29}$ I. Maksimovic, ${ }^{129}$ N. Man, ${ }^{59}$ V. Mandic ${ }^{39}$ V. Mangano, ${ }^{40}$ G. L. Mansell, ${ }^{22}$ M. Manske, ${ }^{18}$ M. Mantovani, ${ }^{26}$ F. Marchesoni, ${ }^{45,37}$ F. Marion, ${ }^{7}$ S. Márka, ${ }^{43}$ Z. Márka, ${ }^{43}$ C. Markakis, ${ }^{11}$ A. S. Markosyan, ${ }^{44}$ E. Maros, ${ }^{1}$ F. Martelli, ${ }^{62,63}$ L. Martellini, ${ }^{59}$ I. W. Martin, ${ }^{40}$ D. V. Martynov, ${ }^{13}$ K. Mason, ${ }^{13}$ A. Masserot, ${ }^{7}$ T. J. Massinger, ${ }^{1}$ M. Masso-Reid, ${ }^{40}$ S. Mastrogiovanni, ${ }^{88,29}$ A. Matas, ${ }^{39}$ F. Matichard ${ }^{13}$ L. Matone, ${ }^{43}$ N. Mavalvala, ${ }^{13}$ R. Mayani, ${ }^{99}$ N. Mazumder, ${ }^{61}$ R. McCarthy, ${ }^{41}$ D. E. McClelland, ${ }^{22}$ S. McCormick, ${ }^{6}$ L. McCuller, ${ }^{13}$ S. C. McGuire, ${ }^{130}$ G. McIntyre, ${ }^{1}$ J. McIver, ${ }^{1}$ D. J. McManus, ${ }^{22}$ T. McRae, ${ }^{22}$ S. T. McWilliams, ${ }^{34,35}$ D. Meacher, ${ }^{79}$ G. D. Meadors, ${ }^{31,9}$ J. Meidam, ${ }^{12}$ E. Mejuto-Villa, ${ }^{8}$ A. Melatos, ${ }^{131}$ G. Mendell, ${ }^{41}$ R. A. Mercer, ${ }^{18}$ E. L. Merilh, ${ }^{41}$ M. Merzougui, ${ }^{59}$ S. Meshkov, ${ }^{1}$ C. Messenger, ${ }^{40}$ C. Messick, ${ }^{79}$ R. Metzdorff, ${ }^{65}$ P. M. Meyers,${ }^{39}$ F. Mezzani, ${ }^{29,88}$ H. Miao,${ }^{50}$ C. Michel,${ }^{23}$ H. Middleton, ${ }^{50}$ E. E. Mikhailov, ${ }^{132}$ L. Milano, ${ }^{72,4}$ A. L. Miller, ${ }^{5}$ A. Miller, ${ }^{88,29}$ B. B. Miller, ${ }^{92}$ J. Miller, ${ }^{13}$ M. Millhouse, ${ }^{91}$ O. Minazzoli, ${ }^{59}$ Y. Minenkov, ${ }^{16}$ J. Ming, ${ }^{31}$ C. Mishra, ${ }^{133}$ S. Mitra, ${ }^{17}$ V. P. Mitrofanov,${ }^{54}$ G. Mitselmakher,${ }^{5}$ R. Mittleman, ${ }^{13}$ A. Moggi, ${ }^{21}$ M. Mohan, ${ }^{26}$ S. R. P. Mohapatra, ${ }^{13}$ M. Montani, ${ }^{62,63}$ B. C. Moore, ${ }^{102}$ C. J. Moore, ${ }^{84}$ D. Moraru, ${ }^{41}$ G. Moreno, ${ }^{41}$ S. R. Morriss, ${ }^{95}$ B. Mours, ${ }^{7}$ C. M. Mow-Lowry, ${ }^{50}$ G. Mueller, ${ }^{5}$ A. W. Muir, ${ }^{93}$ Arunava Mukherjee, ${ }^{9}$ D. Mukherjee, ${ }^{18}$ S. Mukherjee, ${ }^{95}$ N. Mukund, ${ }^{17}$ A. Mullavey, ${ }^{6}$ J. Munch,${ }^{75}$ E. A. M. Muniz, ${ }^{38}$ P. G. Murray, ${ }^{40}$ K. Napier, ${ }^{70}$ I. Nardecchia, ${ }^{27,16}$ L. Naticchioni, ${ }^{88,29}$ R. K. Nayak, ${ }^{134}$ G. Nelemans, ${ }^{58,12}$ T. J. N. Nelson, ${ }^{6}$ M. Neri,,${ }^{51,52}$ M. Nery, ${ }^{9}$ A. Neunzert, ${ }^{113}$ J. M. Newport, ${ }^{114}$ G. Newton, ${ }^{\dagger, 40}$ K. K. Y. Ng, ${ }^{85}$ T. T. Nguyen, ${ }^{22}$ D. Nichols,${ }^{58}$ A. B. Nielsen, ${ }^{9}$ S. Nissanke,${ }^{58,12}$ A. Nitz, ${ }^{9}$ A. Noack, ${ }^{9}$ F. Nocera, ${ }^{26}$ D. Nolting, ${ }^{6}$ M. E. N. Normandin, ${ }^{95}$ L. K. Nuttall, ${ }^{38}$ J. Oberling, ${ }^{41}$ E. Ochsner, ${ }^{18}$ E. Oelker, ${ }^{13}$ G. H. Ogin, ${ }^{105}$ J. J. Oh, ${ }^{123}$ S. H. Oh, ${ }^{123}$ F. Ohme, ${ }^{9}$ M. Oliver, ${ }^{94}$ P. Oppermann, ${ }^{9}$ Richard J. Oram, ${ }^{6}$ B. O'Reilly, ${ }^{6}$ R. Ormiston,${ }^{39}$ L. F. Ortega, ${ }^{5}$ R. O'Shaughnessy, ${ }^{115}$ D. J. Ottaway, ${ }^{75}$ H. Overmier, ${ }^{6}$ B. J. Owen, ${ }^{77}$ A. E. Pace, ${ }^{79}$ J. Page, ${ }^{128}$ M. A. Page, ${ }^{57}$ A. Pai, ${ }^{108}$ S. A. Pai, ${ }^{53}$ J. R. Palamos, ${ }^{64}$ O. Palashov, ${ }^{120}$ C. Palomba, ${ }^{29}$ A. Pal-Singh,${ }^{28}$ H. Pan, ${ }^{80}$ B. Pang,${ }^{56}$ P. T. H. Pang, ${ }^{85}$ C. Pankow, ${ }^{92}$ F. Pannarale, ${ }^{93}$ B. C. Pant, ${ }^{53}$ F. Paoletti, ${ }^{21}$ A. Paoli, ${ }^{26}$ M. A. Papa,${ }^{31,18,9}$ H. R. Paris, ${ }^{44}$ W. Parker, ${ }^{6}$ D. Pascucci, ${ }^{40}$ A. Pasqualetti, ${ }^{26}$ R. Passaquieti, ${ }^{20,21}$ D. Passuello, ${ }^{21}$ B. Patricelli, ${ }^{135,21}$ B. L. Pearlstone, ${ }^{40}$ M. Pedraza, ${ }^{1}$ R. Pedurand ${ }^{23,136}$ L. Pekowsky, ${ }^{38}$ A. Pele, ${ }^{6}$ S. Penn, ${ }^{137}$ C. J. Perez, ${ }^{41}$ A. Perreca, $, 101,87$ L. M. Perri, ${ }^{92}$ H. P. Pfeiffer, ${ }^{104}$ M. Phelps, ${ }^{40}$ O. J. Piccinni, ${ }^{88,29}$ M. Pichot, ${ }^{59}$ F. Piergiovanni, ${ }^{62,63}$ V. Pierro, ${ }^{8}$ G. Pillant,${ }^{26}$ L. Pinard, ${ }^{23}$ I. M. Pinto, ${ }^{8}$ M. Pitkin, ${ }^{40}$ R. Poggiani, ${ }^{20,21}$ P. Popolizio, ${ }^{26}$ E. K. Porter,${ }^{32}$ A. Post, ${ }^{9}$ J. Powell, ${ }^{40}$ J. Prasad, ${ }^{17}$ J. W. W. Pratt,${ }^{30}$ V. Predoi,${ }^{93}$ T. Prestegard, ${ }^{18}$ M. Prijatelj, ${ }^{9}$ M. Principe, ${ }^{8}$ S. Privitera, ${ }^{31}$ R. Prix, ${ }^{9}$ G. A. Prodi, ${ }^{101,87}$ L. G. Prokhorov, ${ }^{54}$ O. Puncken, ${ }^{9}$ M. Punturo, ${ }^{37}$ P. Puppo, ${ }^{29}$ M. Pürrer, ${ }^{31}$ H. Qi ${ }^{18}$ J. Qin, ${ }^{57}$ S. Qiu, ${ }^{126}$ V. Quetschke, ${ }^{95}$ E. A. Quintero, ${ }^{1}$ R. Quitzow-James ${ }^{64}$ F. J. Raab, ${ }^{41}$ D. S. Rabeling, ${ }^{22}$ H. Radkins, ${ }^{41}$ P. Raffai, ${ }^{48}$ S. Raja ${ }^{53}$ C. Rajan, ${ }^{53}$ M. Rakhmanov, ${ }^{95}$ K. E. Ramirez, ${ }^{95}$ P. Rapagnani, ${ }^{88,29}$ V. Raymond, ${ }^{31}$ M. Razzano, ${ }^{20,21}$ J. Read, ${ }^{25}$ T. Regimbau, ${ }^{59}$ L. Rei, ${ }^{52}$ S. Reid, ${ }^{55}$

D. H. Reitze ${ }^{1,5}$ H. Rew, ${ }^{132}$ S. D. Reyes,${ }^{38}$ F. Ricci, ${ }^{88,29}$ P. M. Ricker,${ }^{11}$ S. Rieger, ${ }^{9}$ K. Riles,${ }^{113}$ M. Rizzo, ${ }^{115}$ N. A. Robertson, ${ }^{1,40}$ R. Robie, ${ }^{40}$ F. Robinet, ${ }^{24}$ A. Rocchi, ${ }^{16}$ L. Rolland, ${ }^{7}$ J. G. Rollins, ${ }^{1}$ V. J. Roma, ${ }^{64}$ R. Romano, ${ }^{3,4}$ 
C. L. Romel, ${ }^{41}$ J. H. Romie, ${ }^{6}$ D. Rosińska, ${ }^{138,49}$ M. P. Ross, ${ }^{139}$ S. Rowan, ${ }^{40}$ A. Rüdiger, ${ }^{9}$ P. Ruggi, ${ }^{26}$ K. Ryan, ${ }^{41}$ M. Rynge ${ }^{99}$ S. Sachdev, ${ }^{1}$ T. Sadecki, ${ }^{41}$ L. Sadeghian, ${ }^{18}$ M. Sakellariadou, ${ }^{140}$ L. Salconi,${ }^{26}$ M. Saleem, ${ }^{108}$ F. Salemi,${ }^{9}$ A. Samajdar, ${ }^{134}$ L. Sammut, ${ }^{126}$ L. M. Sampson, ${ }^{92}$ E. J. Sanchez, ${ }^{1}$ V. Sandberg, ${ }^{41}$ B. Sandeen, ${ }^{92}$ J. R. Sanders, ${ }^{38}$ B. Sassolas, ${ }^{23}$ B. S. Sathyaprakash, ${ }^{79}$ P. R. Saulson, ${ }^{38}$ O. Sauter, ${ }^{113}$ R. L. Savage, ${ }^{41}$ A. Sawadsky, ${ }^{19}$ P. Schale, ${ }^{64}$ J. Scheuer, ${ }^{92}$ E. Schmidt, ${ }^{30}$ J. Schmidt, ${ }^{9}$ P. Schmidt, ${ }^{1,58}$ R. Schnabel, ${ }^{28}$ R. M. S. Schofield, ${ }^{64}$ A. Schönbeck, ${ }^{28}$ E. Schreiber, ${ }^{9}$ D. Schuette, ${ }^{9,19}$ B. W. Schulte, ${ }^{9}$ B. F. Schutz, ${ }^{93,9}$ S. G. Schwalbe, ${ }^{30}$ J. Scott ${ }^{40}$ S. M. Scott, ${ }^{22}$ E. Seidel, ${ }^{11}$ D. Sellers, ${ }^{6}$ A. S. Sengupta, ${ }^{141}$ D. Sentenac, ${ }^{26}$ V. Sequino, ${ }^{27,16}$ A. Sergeev, ${ }^{120}$ D. A. Shaddock, ${ }^{22}$ T. J. Shaffer, ${ }^{41}$ A. A. Shah, ${ }^{128}$ M. S. Shahriar, ${ }^{92}$ L. Shao, ${ }^{31}$ B. Shapiro, ${ }^{44}$ P. Shawhan, ${ }^{69}$ A. Sheperd, ${ }^{18}$ D. H. Shoemaker, ${ }^{13}$ D. M. Shoemaker, ${ }^{70}$ K. Siellez, ${ }^{70}$ X. Siemens, ${ }^{18}$ M. Sieniawska, ${ }^{49}$ D. Sigg, ${ }^{41}$ A. D. Silva, ${ }^{14}$ A. Singer, ${ }^{1}$ L. P. Singer ${ }^{73}$ A. Singh,${ }^{31,9,19}$ R. Singh, ${ }^{2}$ A. Singhal, ${ }^{15,29}$ A. M. Sintes, ${ }^{94}$ B. J. J. Slagmolen, ${ }^{22}$ B. Smith, ${ }^{6}$ J. R. Smith, ${ }^{25}$ R. J. E. Smith, ${ }^{1}$ E. J. Son, ${ }^{123}$ J. A. Sonnenberg, ${ }^{18}$ B. Sorazu, ${ }^{40}$ F. Sorrentino, ${ }^{52}$ T. Souradeep, ${ }^{17}$ A. P. Spencer, ${ }^{40}$ A. K. Srivastava, ${ }^{97}$ A. Staley, ${ }^{43}$ M. Steinke, ${ }^{9}$ J. Steinlechner ${ }^{40,28}$ S. Steinlechner, ${ }^{28}$ D. Steinmeyer, ${ }^{9,19}$ B. C. Stephens, ${ }^{18}$ R. Stone, ${ }^{95}$ K. A. Strain, ${ }^{40}$ G. Stratta, ${ }^{62,63}$ S. E. Strigin, ${ }^{54}$ R. Sturani, ${ }^{142}$ A. L. Stuver, ${ }^{6}$ T. Z. Summerscales, ${ }^{143}$ L. Sun, ${ }^{131}$ S. Sunil, ${ }^{97}$ P. J. Sutton, ${ }^{93}$ B. L. Swinkels, ${ }^{26}$ M. J. Szczepańczyk ${ }^{30}$ M. Tacca, ${ }^{32}$ D. Talukder, ${ }^{64}$ D. B. Tanner, ${ }^{5}$ M. Tápai, ${ }^{109}$ A. Taracchini, ${ }^{31}$ J. A. Taylor ${ }^{128}$ R. Taylor, ${ }^{1}$ T. Theeg, ${ }^{9}$ E. G. Thomas, ${ }^{50}$ M. Thomas, ${ }^{6}$ P. Thomas, ${ }^{41}$ K. A. Thorne, ${ }^{6}$ K. S. Thorne, ${ }^{56}$ E. Thrane, ${ }^{126}$ S. Tiwari, ${ }^{15,87}$ V. Tiwari, ${ }^{93}$ K. V. Tokmakov, ${ }^{117}$ K. Toland, ${ }^{40}$ M. Tonelli, ${ }^{20,21}$ Z. Tornasi ${ }^{40}$ C. I. Torrie, ${ }^{1}$ D. Töyrä, ${ }^{50}$ F. Travasso, ${ }^{26,37}$ G. Traylor, ${ }^{6}$ D. Trifirò, ${ }^{10}$ J. Trinastic, ${ }^{5}$ M. C. Tringali, ${ }^{101,87}$ L. Trozzo, ${ }^{144,21}$ K. W. Tsang, ${ }^{12}$ M. Tse, ${ }^{13}$ R. Tso, ${ }^{1}$ D. Tuyenbayev, ${ }^{95}$ K. Ueno, ${ }^{18}$ D. Ugolini, ${ }^{145}$ C. S. Unnikrishnan, ${ }^{111}$ A. L. Urban, ${ }^{1}$ S. A. Usman, ${ }^{93}$

K. Vahi,${ }^{99}$ H. Vahlbruch, ${ }^{19}$ G. Vajente, ${ }^{1}$ G. Valdes,${ }^{95}$ M. Vallisneri ${ }^{56}$ N. van Bakel, ${ }^{12}$ M. van Beuzekom, ${ }^{12}$ J. F. J. van den Brand, ${ }^{68,12}$ C. Van Den Broeck, ${ }^{12}$ D. C. Vander-Hyde, ${ }^{38}$ L. van der Schaaf, ${ }^{12}$ J. V. van Heijningen, ${ }^{12}$ A. A. van Veggel, ${ }^{40}$ M. Vardaro, ${ }^{46,47}$ V. Varma, ${ }^{56}$ S. Vass, ${ }^{1}$ M. Vasúth, ${ }^{42}$ A. Vecchio, ${ }^{50}$ G. Vedovato, ${ }^{47}$ J. Veitch, ${ }^{50}$

P. J. Veitch ${ }^{75}$ K. Venkateswara, ${ }^{139}$ G. Venugopalan, ${ }^{1}$ D. Verkindt, ${ }^{7}$ F. Vetrano, ${ }^{62,63}$ A. Viceré, ${ }^{62,63}$ A. D. Viets, ${ }^{18}$ S. Vinciguerra, ${ }^{50}$ D. J. Vine, ${ }^{55}$ J.-Y. Vinet, ${ }^{59}$ S. Vitale, ${ }^{13}$ T. Vo, ${ }^{38}$ H. Vocca,${ }^{36,37}$ C. Vorvick, ${ }^{41}$ D. V. Voss, ${ }^{5}$ W. D. Vousden,${ }^{50}$ S. P. Vyatchanin, ${ }^{54}$ A. R. Wade, ${ }^{1}$ L. E. Wade ${ }^{78}$ M. Wade, ${ }^{78}$ R. Walet, ${ }^{12}$ M. Walker, ${ }^{2}$ L. Wallace, ${ }^{1}$ S. Walsh, ${ }^{18}$ G. Wang,,${ }^{15,63}$ H. Wang, ${ }^{50}$ J. Z. Wang, ${ }^{79}$ M. Wang, ${ }^{50}$ Y.-F. Wang, ${ }^{85}$ Y. Wang, ${ }^{57}$ R. L. Ward, ${ }^{22}$ J. Warner,${ }^{41}$ M. Was, ${ }^{7}$ J. Watchi,${ }^{89}$ B. Weaver, ${ }^{41}$ L.-W. Wei, ${ }^{9,19}$ M. Weinert, ${ }^{9}$ A. J. Weinstein, ${ }^{1}$ R. Weiss,${ }^{13}$ L. Wen, ${ }^{57}$ E. K. Wessel, ${ }^{11}$ P. Weßels, ${ }^{9}$ T. Westphal, ${ }^{9}$ K. Wette, ${ }^{9}$ J. T. Whelan, ${ }^{115}$ B. F. Whiting, ${ }^{5}$ C. Whittle, ${ }^{126}$ D. Williams,${ }^{40}$ R. D. Williams, ${ }^{1}$ A. R. Williamson, ${ }^{115}$ J. L. Willis,${ }^{146}$

B. Willke, ${ }^{19,9}$ M. H. Wimmer, ${ }^{9,19}$ W. Winkler, ${ }^{9}$ C. C. Wipf, ${ }^{1}$ H. Wittel,${ }^{9,19}$ G. Woan, ${ }^{40}$ J. Woehler, ${ }^{9}$ J. Wofford, ${ }^{115}$ K. W. K. Wong, ${ }^{85}$ J. Worden, ${ }^{41}$ J. L. Wright, ${ }^{40}$ D. S. Wu, ${ }^{9}$ G. Wu, ${ }^{6}$ W. Yam, ${ }^{13}$ H. Yamamoto, ${ }^{1}$ C. C. Yancey, ${ }^{69}$ M. J. Yap, ${ }^{22}$ Hang Yu, ${ }^{13}$ Haocun Yu, ${ }^{13}$ M. Yvert, ${ }^{7}$ A. Zadrożny, ${ }^{124}$ M. Zanolin, ${ }^{30}$ T. Zelenova, ${ }^{26}$ J.-P. Zendri, ${ }^{47}$ M. Zevin, ${ }^{92}$ L. Zhang, ${ }^{1}$ M. Zhang, ${ }^{132}$ T. Zhang, ${ }^{40}$ Y.-H. Zhang, ${ }^{115}$ C. Zhao, ${ }^{57}$ M. Zhou, ${ }^{92}$ Z. Zhou, ${ }^{92}$ S. J. Zhu, ${ }^{31,9}$ X. J. Zhu, ${ }^{57}$

M. E. Zucker, ${ }^{1,13}$ and J. Zweizig ${ }^{1}$

(LIGO Scientific Collaboration and Virgo Collaboration)

and D. P. Anderson ${ }^{147}$

${ }^{1}$ LIGO, California Institute of Technology, Pasadena, California 91125, USA

${ }^{2}$ Louisiana State University, Baton Rouge, Louisiana 70803, USA

${ }^{3}$ Università di Salerno, Fisciano, I-84084 Salerno, Italy

${ }^{4}$ INFN, Sezione di Napoli, Complesso Universitario di Monte S.Angelo, I-80126 Napoli, Italy

${ }^{5}$ University of Florida, Gainesville, Florida 32611, USA

${ }^{6}$ LIGO Livingston Observatory, Livingston, Louisiana 70754, USA

${ }^{7}$ Laboratoire d'Annecy-le-Vieux de Physique des Particules (LAPP), Université Savoie Mont Blanc, CNRS/IN2P3, F-74941 Annecy, France

${ }^{8}$ University of Sannio at Benevento, I-82100 Benevento, Italy and INFN, Sezione di Napoli, I-80100 Napoli, Italy

${ }^{9}$ Albert-Einstein-Institut, Max-Planck-Institut für Gravitationsphysik, D-30167 Hannover, Germany

${ }^{10}$ The University of Mississippi, University, Mississippi 38677, USA

${ }^{11}$ NCSA, University of Illinois at Urbana-Champaign, Urbana, Illinois 61801, USA

${ }^{12}$ Nikhef, Science Park, 1098 XG Amsterdam, Netherlands

${ }^{13}$ LIGO, Massachusetts Institute of Technology, Cambridge, Massachusetts 02139, USA 
${ }^{14}$ Instituto Nacional de Pesquisas Espaciais, 12227-010 São José dos Campos, São Paulo, Brazil

${ }^{15}$ Gran Sasso Science Institute (GSSI), I-67100 L'Aquila, Italy

${ }^{16}$ INFN, Sezione di Roma Tor Vergata, I-00133 Roma, Italy

${ }^{17}$ Inter-University Centre for Astronomy and Astrophysics, Pune 411007, India

${ }^{18}$ University of Wisconsin-Milwaukee, Milwaukee, Wisconsin 53201, USA

${ }^{19}$ Leibniz Universität Hannover, D-30167 Hannover, Germany

${ }^{20}$ Università di Pisa, I-56127 Pisa, Italy

${ }^{21}$ INFN, Sezione di Pisa, I-56127 Pisa, Italy

${ }^{22}$ OzGrav, Australian National University, Canberra, Australian Capital Territory 0200, Australia

${ }^{23}$ Laboratoire des Matériaux Avancés (LMA), CNRS/IN2P3, F-69622 Villeurbanne, France

${ }^{24}$ LAL, Univ. Paris-Sud, CNRS/IN2P3, Université Paris-Saclay, F-91898 Orsay, France

${ }^{25}$ California State University Fullerton, Fullerton, California 92831, USA

${ }^{26}$ European Gravitational Observatory (EGO), I-56021 Cascina, Pisa, Italy

${ }^{27}$ Università di Roma Tor Vergata, I-00133 Roma, Italy

${ }^{28}$ Universität Hamburg, D-22761 Hamburg, Germany

${ }^{29}$ INFN, Sezione di Roma, I-00185 Roma, Italy

${ }^{30}$ Embry-Riddle Aeronautical University, Prescott, Arizona 86301, USA

${ }^{31}$ Albert-Einstein-Institut, Max-Planck-Institut für Gravitationsphysik, D-14476 Potsdam-Golm, Germany

${ }^{32}$ APC, AstroParticule et Cosmologie, Université Paris Diderot, CNRS/IN2P3, CEA/Irfu, Observatoire de

Paris, Sorbonne Paris Cité, F-75205 Paris Cedex 13, France

${ }^{33}$ Korea Institute of Science and Technology Information, Daejeon 34141, Korea

${ }^{34}$ West Virginia University, Morgantown, West Virginia 26506, USA

${ }^{35}$ Center for Gravitational Waves and Cosmology, West Virginia University, Morgantown, West Virginia 26505, USA

${ }^{36}$ Università di Perugia, I-06123 Perugia, Italy

${ }^{37}$ INFN, Sezione di Perugia, I-06123 Perugia, Italy

${ }^{38}$ Syracuse University, Syracuse, New York 13244, USA

${ }^{39}$ University of Minnesota, Minneapolis, Minnesota 55455, USA

${ }^{40}$ SUPA, University of Glasgow, Glasgow G12 8QQ, United Kingdom

${ }^{41}$ LIGO Hanford Observatory, Richland, Washington 99352, USA

${ }^{42}$ Wigner RCP, RMKI, H-1121 Budapest, Konkoly Thege Miklós út 29-33, Hungary

${ }^{43}$ Columbia University, New York, New York 10027, USA

${ }^{44}$ Stanford University, Stanford, California 94305, USA

${ }^{45}$ Università di Camerino, Dipartimento di Fisica, I-62032 Camerino, Italy

${ }^{46}$ Università di Padova, Dipartimento di Fisica e Astronomia, I-35131 Padova, Italy

${ }^{47}$ INFN, Sezione di Padova, I-35131 Padova, Italy

${ }^{48}$ MTA Eötvös University, "Lendulet” Astrophysics Research Group, Budapest 1117, Hungary

${ }^{49}$ Nicolaus Copernicus Astronomical Center, Polish Academy of Sciences, 00-716 Warsaw, Poland

${ }^{50}$ University of Birmingham, Birmingham B15 2TT, United Kingdom

${ }^{51}$ Università degli Studi di Genova, I-16146 Genova, Italy

${ }^{52}$ INFN, Sezione di Genova, I-16146 Genova, Italy

${ }^{53}$ RRCAT, Indore Madhya Pradesh 452013, India

${ }^{54}$ Faculty of Physics, Lomonosov Moscow State University, Moscow 119991, Russia

${ }^{55}$ SUPA, University of the West of Scotland, Paisley PA1 2BE, United Kingdom

${ }^{56}$ Caltech CaRT, Pasadena, California 91125, USA

${ }^{57} \mathrm{OzGrav}$, University of Western Australia, Crawley, Western Australia 6009, Australia

${ }^{58}$ Department of Astrophysics/IMAPP, Radboud University Nijmegen,

P.O. Box 9010, 6500 GL Nijmegen, Netherlands

${ }^{59}$ Artemis, Université Côte d'Azur, Observatoire Côte d'Azur, CNRS, CS 34229, F-06304 Nice Cedex 4, France

${ }^{60}$ Institut de Physique de Rennes, CNRS, Université de Rennes 1, F-35042 Rennes, France

${ }^{61}$ Washington State University, Pullman, Washington 99164, USA

${ }^{62}$ Università degli Studi di Urbino 'Carlo Bo', I-61029 Urbino, Italy

${ }^{63}$ INFN, Sezione di Firenze, I-50019 Sesto Fiorentino, Firenze, Italy

${ }^{64}$ University of Oregon, Eugene, Oregon 97403, USA

${ }^{65}$ Laboratoire Kastler Brossel, UPMC-Sorbonne Universités, CNRS, ENS-PSL Research University, Collège de France, F-75005 Paris, France

${ }^{66}$ Carleton College, Northfield, Minnesota 55057, USA

${ }^{67}$ Astronomical Observatory Warsaw University, 00-478 Warsaw, Poland

${ }^{68} \mathrm{VU}$ University Amsterdam, $1081 \mathrm{HV}$ Amsterdam, Netherlands 
${ }^{69}$ University of Maryland, College Park, Maryland 20742, USA

${ }^{70}$ Center for Relativistic Astrophysics and School of Physics, Georgia Institute of Technology, Atlanta, Georgia 30332, USA

${ }^{71}$ Université Claude Bernard Lyon 1, F-69622 Villeurbanne, France

${ }^{72}$ Università di Napoli 'Federico II', Complesso Universitario di Monte S.Angelo, I-80126 Napoli, Italy

${ }^{73}$ NASA Goddard Space Flight Center, Greenbelt, Maryland 20771, USA

${ }^{74}$ RESCEU, University of Tokyo, Tokyo 113-0033, Japan.

${ }^{75} \mathrm{OzGrav}$, University of Adelaide, Adelaide, South Australia 5005, Australia

${ }^{76}$ Tsinghua University, Beijing 100084, China

${ }^{77}$ Texas Tech University, Lubbock, Texas 79409, USA

${ }^{78}$ Kenyon College, Gambier, Ohio 43022, USA

${ }^{79}$ The Pennsylvania State University, University Park, Pennsylvania 16802, USA

${ }^{80}$ National Tsing Hua University, Hsinchu City, 30013 Taiwan, Republic of China

${ }^{81}$ Charles Sturt University, Wagga Wagga, New South Wales 2678, Australia

${ }^{82}$ University of Chicago, Chicago, Illinois 60637, USA

${ }^{83}$ Pusan National University, Busan 46241, Korea

${ }^{84}$ University of Cambridge, Cambridge CB2 1TN, United Kingdom

${ }^{85}$ The Chinese University of Hong Kong, Shatin, NT, Hong Kong

${ }^{86}$ INAF, Osservatorio Astronomico di Padova, Vicolo dell'Osservatorio 5, I-35122 Padova, Italy

${ }^{87}$ INFN, Trento Institute for Fundamental Physics and Applications, I-38123 Povo, Trento, Italy

${ }^{88}$ Università di Roma 'La Sapienza', I-00185 Roma, Italy

${ }^{89}$ Université Libre de Bruxelles, Brussels 1050, Belgium

${ }^{90}$ Sonoma State University, Rohnert Park, California 94928, USA

${ }^{91}$ Montana State University, Bozeman, Montana 59717, USA

${ }^{92}$ Center for Interdisciplinary Exploration \& Research in Astrophysics (CIERA), Northwestern University, Evanston, Illinois 60208, USA

${ }^{93}$ Cardiff University, Cardiff CF24 3AA, United Kingdom

${ }^{94}$ Universitat de les Illes Balears, IAC3-IEEC, E-07122 Palma de Mallorca, Spain

${ }^{95}$ The University of Texas Rio Grande Valley, Brownsville, Texas 78520, USA

${ }^{96}$ Bellevue College, Bellevue, Washington 98007, USA

${ }^{97}$ Institute for Plasma Research, Bhat, Gandhinagar 382428, India

${ }^{98}$ The University of Sheffield, Sheffield S10 2TN, United Kingdom

${ }^{99}$ University of Southern California Information Sciences Institute, Marina Del Rey, California 90292, USA

${ }^{100}$ California State University, Los Angeles, 5151 State University Dr, Los Angeles, California 90032, USA

${ }^{101}$ Università di Trento, Dipartimento di Fisica, I-38123 Povo, Trento, Italy

${ }^{102}$ Montclair State University, Montclair, New Jersey 07043, USA

${ }^{103}$ National Astronomical Observatory of Japan, 2-21-1 Osawa, Mitaka, Tokyo 181-8588, Japan

${ }^{104}$ Canadian Institute for Theoretical Astrophysics, University of Toronto,

Toronto, Ontario M5S 3H8, Canada

${ }^{105}$ Whitman College, 345 Boyer Avenue, Walla Walla, Washington 99362 USA

${ }^{106}$ School of Mathematics, University of Edinburgh, Edinburgh EH9 3FD, United Kingdom

${ }^{107}$ University and Institute of Advanced Research, Gandhinagar Gujarat 382007, India

${ }^{108}$ IISER-TVM, CET Campus, Trivandrum Kerala 695016, India

${ }^{109}$ University of Szeged, Dóm tér 9, Szeged 6720, Hungary

${ }^{110}$ International Centre for Theoretical Sciences, Tata Institute of Fundamental Research, Bengaluru 560089, India

${ }^{111}$ Tata Institute of Fundamental Research, Mumbai 400005, India

${ }^{112}$ INAF, Osservatorio Astronomico di Capodimonte, I-80131 Napoli, Italy

${ }^{113}$ University of Michigan, Ann Arbor, Michigan 48109, USA

${ }^{114}$ American University, Washington, D.C. 20016, USA

${ }^{115}$ Rochester Institute of Technology, Rochester, New York 14623, USA

${ }^{116}$ University of Biat ystok, 15-424 Biatystok, Poland

${ }^{117}$ SUPA, University of Strathclyde, Glasgow G1 1XQ, United Kingdom

${ }^{118}$ University of Southampton, Southampton SO17 1BJ, United Kingdom

${ }^{119}$ University of Washington Bothell, 18115 Campus Way NE, Bothell, Washington 98011, USA

${ }^{120}$ Institute of Applied Physics, Nizhny Novgorod, 603950, Russia

${ }^{121}$ Seoul National University, Seoul 08826, Korea

${ }^{122}$ Inje University Gimhae, South Gyeongsang 50834, Korea

${ }^{123}$ National Institute for Mathematical Sciences, Daejeon 34047, Korea 
${ }^{124}$ NCBJ, 05-400 Świerk-Otwock, Poland

${ }^{125}$ Institute of Mathematics, Polish Academy of Sciences, 00656 Warsaw, Poland

${ }^{126}$ OzGrav, School of Physics \& Astronomy, Monash University, Clayton 3800, Victoria, Australia

${ }^{127}$ Hanyang University, Seoul 04763, Korea

${ }^{128}$ NASA Marshall Space Flight Center, Huntsville, Alabama 35811, USA

${ }^{129}$ ESPCI, CNRS, F-75005 Paris, France

${ }^{130}$ Southern University and A\&M College, Baton Rouge, Louisiana 70813, USA

${ }^{131}$ OzGrav, University of Melbourne, Parkville, Victoria 3010, Australia

${ }^{132}$ College of William and Mary, Williamsburg, Virginia 23187, USA

${ }^{133}$ Indian Institute of Technology Madras, Chennai 600036, India

${ }^{134}$ IISER-Kolkata, Mohanpur, West Bengal 741252, India

${ }^{135}$ Scuola Normale Superiore, Piazza dei Cavalieri 7, I-56126 Pisa, Italy

${ }^{136}$ Université de Lyon, F-69361 Lyon, France

${ }^{137}$ Hobart and William Smith Colleges, Geneva, New York 14456, USA

${ }^{138}$ Janusz Gil Institute of Astronomy, University of Zielona Góra, 65-265 Zielona Góra, Poland

${ }^{139}$ University of Washington, Seattle, Washington 98195, USA

${ }^{140}$ King's College London, University of London, London WC2R 2LS, United Kingdom

${ }^{141}$ Indian Institute of Technology, Gandhinagar Ahmedabad Gujarat 382424, India

${ }^{142}$ International Institute of Physics, Universidade Federal do Rio Grande do Norte, Natal RN 59078-970, Brazil

${ }^{143}$ Andrews University, Berrien Springs, Michigan 49104, USA

${ }^{144}$ Università di Siena, I-53100 Siena, Italy

${ }^{145}$ Trinity University, San Antonio, Texas 78212, USA

${ }^{146}$ Abilene Christian University, Abilene, Texas 79699, USA

${ }^{147}$ University of California at Berkeley, Berkeley, California 94720, USA

${ }^{\dagger}$ Deceased. 